\title{
Evolution and epigenetic regulation of RNA-mediated duplicated genes in Arabidopsis
}

\author{
Dissertation \\ For the award of the degree \\ “Doctor rerum naturalium” (Dr.rer.nat.) \\ of the Georg August University Göttingen \\ within the doctoral program: Biology \\ of the Georg-August University School of Science (GAUSS)
}

submitted by

Ahmed Mahmoud Abdelsamad Abdrabou

from Cairo, Egypt

Göttingen, 2015 


\section{Thesis Committee}

Prof. Dr. Christiane Gatz

(Department of Plant Molecular Biology and Physiology / Schwann-SchleidenResearch-Center for Molecular Cell Biology, University of Göttingen)

Prof. Dr. Stefan Jakobs

(Department of Nanobiophotonics/ Structure and Dynamics of Mitochondria Group, Max Planck Institute for Biophysical Chemistry and University of Göttingen)

Dr. Ales Pecinka

(Department of Plant Breeding and Genetics / Genome and Epigenome Evolution Group, Max Planck Institute for Plant Breeding Research)

\section{Members of the Examination Board}

Reviewer: Prof. Dr. Christiane Gatz

(Department of Plant Molecular Biology and Physiology / Schwann-SchleidenResearch-Center for Molecular Cell Biology)

Second Reviewer: Prof. Dr. Stefan Jakobs

(Department of Nanobiophotonics/ Structure and Dynamics of Mitochondria Group, Max Planck Institute for Biophysical Chemistry and University of Göttingen)

\section{Further members of the Examination Board:}

Prof. Dr. Gregor Bucher

(Department of Developmental Biology / Göttingen Center for Molecular Biosciences)

Prof. Dr. André Fischer

(Department of Psychiatry and Psychotherapy, University Medical Center)

Prof. Dr. Volker Lipka

(Department of Plant Cell Biology / Schwann-Schleiden-Research-Center for Molecular Cell Biology)

Prof. Dr. Burkhard Morgenstern

(Department of Bioinformatics/ Institute for Microbiology and Genetics)

Date of the oral examination: 15. 06. 2015 


\section{TABLE of CONTENTS}

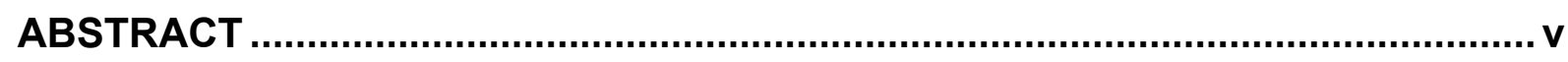

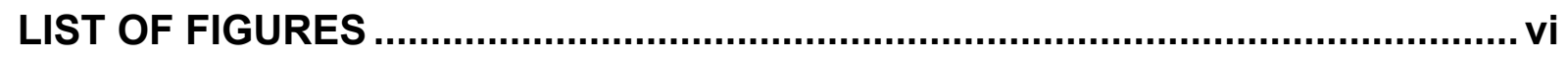

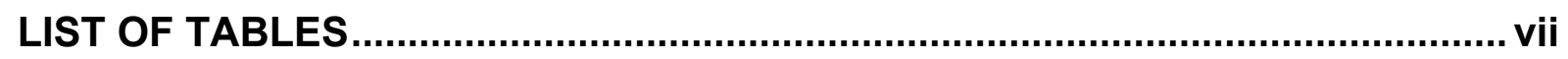

LIST OF ABBREVIATIONS .......................................................................... viii

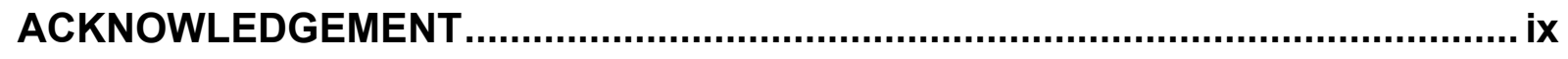

DECLARATION.........................................................................................

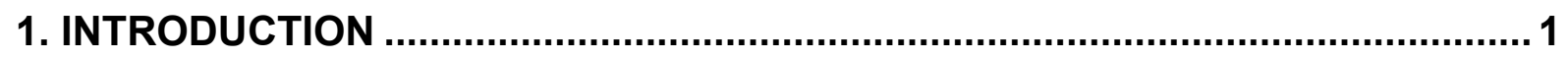

2. RESULTS ............................................................................................... 5

2.1 Annotation of $A$. thaliana retrogenes by a novel retrogene annotation tool (RAT) 5

2.2 A. thaliana retrogenes are capable of repeated retroposition and occur in gene-

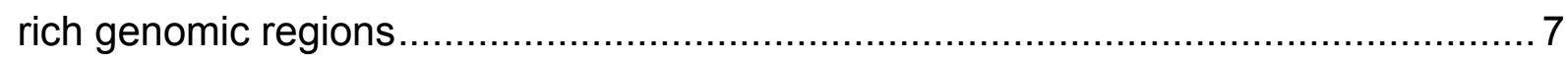

2.3 Retrogenes are derived from highly transcribed parental genes and are transcribed preferentially by novel promoters............................................ 101

2.4 A. thaliana retrogenes are transcribed in male gametes .............................. 13

2.5 Retrogenes are deficient for transcription-permissive chromatin marks in leaf

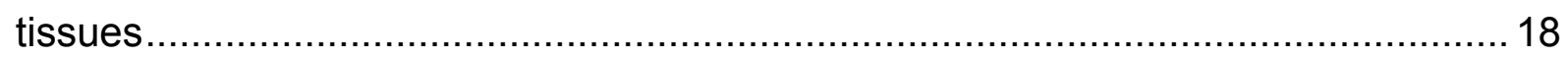

2.6 Gain of transcription factor binding sites facilitates PCR11 retrogene sperm-

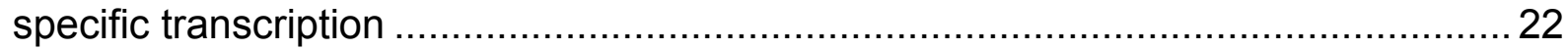

2.7 Improving gene structure annotation of $A$. lyrata genome using RNA-seq data .. 25

2.8 Fast emergence of Arabidopsis retrogenes revealed by interspecies comparison

2.9 Arabidopsis retrogenes and transposable elements share amplification mechanism but not chromosomal location and transcriptional regulation ................29

$2.10 N R P D 2 E 2^{A l y \_M N 47}$ : an unusual retrogene in $A$. lyrata genome ........................... 33

2.11 Arabidopsis retrogene targeting and the origin of their introns ....................... 36

2.12 Introns increase stability of retrogenes transcripts ................................. 39

3. DISCUSSION

3.1 The novel bioinformatic retrogene annotation tool (RAT) proved useful for identification of retrogenes across plant genomes 
3.1.1 Annotation of retrogenes in A. thaliana genome (TAIR10)

3.1.2 Identification of $A$. lyrata retrogenes using the newly developed genome annotation

3.2 Fast evolutionary emergence of Arabidopsis retrogenes .................................. 43

3.3 Multiple and repeated retropositions in Arabidopsis .......................................... 45

3.4 Arabidopsis retrogenes are transcribed via newly acquired promoters ............... 46

3.5 Retrogenes are preferentially up regulated in pollen ..................................... 47

3.6 Arabidopsis retrogenes and retrotransposons share retroposition mechanism but

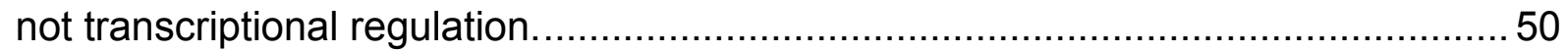

3.7 Arabidopsis natural in planta retrogene targeting ......................................... 50

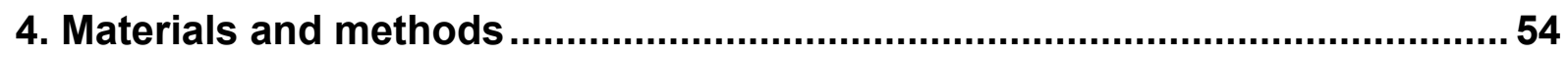

4.1 Defining the minimum length of poly(A)-tail in Arabidopsis genome. ................. 54

4.2 Genome-wide transcription and mRNA half-life analysis................................ 55

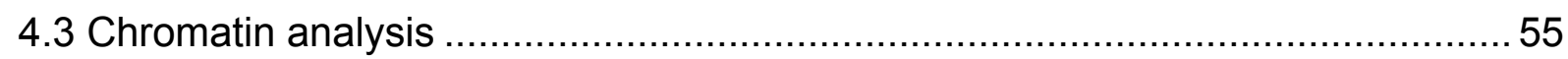

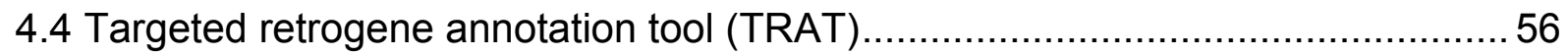

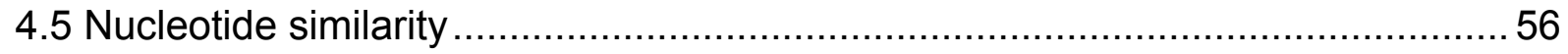

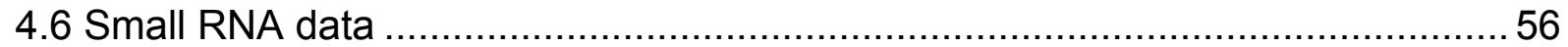

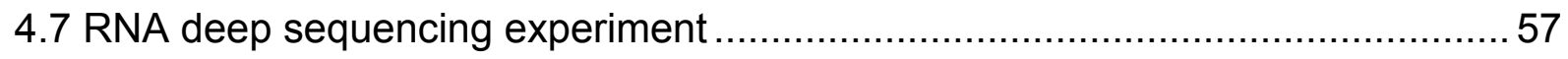

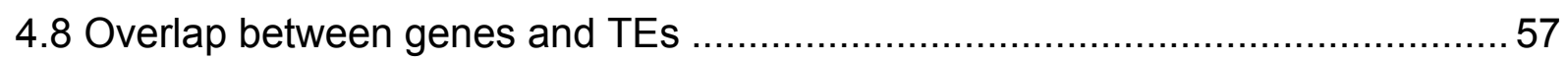

5. APPENDICES

5.1 Appendix A. Comprehensive list of $A$. thaliana retrogenes.............................. 58

5.2 Appendix B. Association of genes with epigenetic marks................................... 64

5.3 Appendix C. Robust Multiarray Averaging (gcRMA) values ..............................65

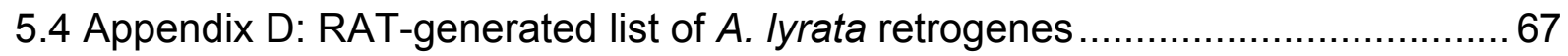

5.5 Appendix E: Interspecies comparison of NRPD2E2 DNA sequence..................70

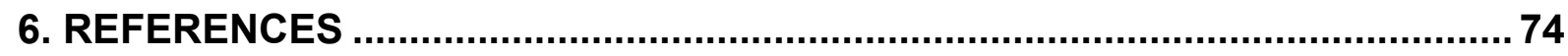

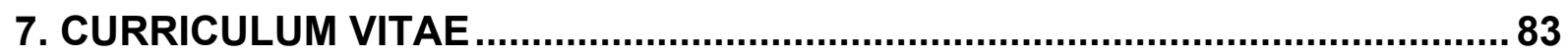




\section{ABSTRACT}

Gene duplications allow for protein functional diversification and accelerate genome evolution. Occasionally, the transposon amplification machinery reversetranscribes mRNA of a gene, integrates it into the genome and forms an RNAduplicated gene copy, the retrogene. Although retrogenes have been found in plants, their biology, evolution and epigenetic regulation are poorly understood. We developed a novel bioinformatic retrogene annotation tool (RAT) to screen Arabidopsis genomes for retrogenes. We identified 251 (216 novel) and 168 retrogenes in Arabidopsis thaliana and Arabidopsis lyrata, corresponding to $1 \%$ and $0.5 \%$ of protein coding genes respectively. Based on our findings, we calculated emergence rate of five to ten retrogenes per million years, which is at least ten times faster than previously estimated. Most of retrogenes were randomly integrated away from their parental gene loci; however, some showed targeted integration replacing their parental genes. Therefore, we developed a bioinformatic targeted retrogene annotation tool (TRAT) to screen Arabidopsis genomes for these rare cases. To our knowledge, we report the first natural in planta retrogene targeting events.

Arabidopsis retrogenes are derived from ubiquitously transcribed parents and reside in gene rich chromosomal regions, depleted of transposons. Unlike transposon regulation, we found retrogenes and their parents to be targets of gene-specific regulatory $21 \mathrm{nt}$ sRNAs rather than transposon-specific $24 \mathrm{nt}$ sRNAs. Retrogene expression levels are relatively low, but significantly higher than that of transposable elements. Approximately $25 \%$ of retrogenes are co-transcribed with their parents, and $3 \%$ with head-to-head oriented neighbors. This suggests transcription by novel or modified promoters for at least $72 \%$ of $A$. thaliana retrogenes. Many retrogenes reach their transcription maximum in pollen, the tissue analogous to animal spermatocytes where up-regulation of retrogenes has previously been found. This implies an evolutionarily conserved mechanism leading to this transcription pattern of RNA-duplicated genes. During transcriptional repression, retrogenes are depleted of permissive chromatin marks without an obvious enrichment for repressive modifications. However, this pattern is common to many other pollen-transcribed genes independent of their evolutionary origin. Hence, retroposition plays role in plant genome evolution and developmental transcription pattern of retrogenes suggests analogous control of RNA-duplicated genes in plants and animals. 


\section{LIST OF FIGURES}

Figure 1. Annotation of $A$. thaliana retrogenes using the RAT tool........................ 6

Figure 2. Genomic features of $A$. thaliana retrogenes ..................................... 9

Figure 3. Retrogenes are driven by novel promoters ..................................... 10

Figure 4. Retrogenes are transcriptionally up-regulated in pollen ......................... 15

Figure 5. Arabidopsis retrogenes are expressed in pollen ................................... 17

Figure 6. Expression correlations between pollen and chromatin mutants .............. 19

Figure 7. Chromatin control of pollen-specific gene transcription ......................... 21

Figure 8. Chromatin control of pollen-specific gene expression ............................. 22

Figure 9. Gain of pollen-specific transcription by PCR11 retrogene ........................ 24

Figure 10. Enhancement of $A$. lyrata gene models using RNA-seq .......................26

Figure 11. Novel identification of $A$. lyrata retrogenes and the relatively recent

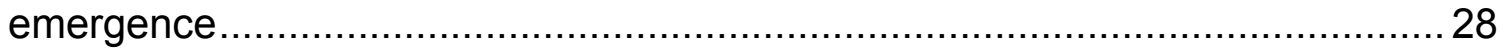

Figure 12. Distinctive chromosomal location of retrogenes and TEs........................30

Figure 13. Retrogenes are not integrated, expressed or regulated like TEs ............. 32

Figure 14. NRPD2E2 ${ }^{\text {Aly_MN47 }}$ retrogene targeting event ....................................... 35

Figure 15. Identification of natural retrogene targeting events using TRAT ..............37

Figure 16. Introns increase half-life of retrogenes transcripts...............................40

Figure 17. Evolution of NRPD2E2 gene in A. lyrata. .......................................... 51

Figure 18. Defining the minimum length of non-random poly(A)-tail in Arabidopsis genome 


\section{LIST OF TABLES}

Table 1. Repeated retroposition events in A. thaliana ..................................... 8

Table 2. Comparison of $A$. lyrata annotation (version 2) to version 1 and TAIR10 .... 26

Table 3. Total (conserved) retrogenes and parental genes identified by RAT tool.... 29

Table 4. Repeated retroposition events in A. Iyrata .......................................... 29

Table 5. Natural retrogene targeting events .............................................. 37

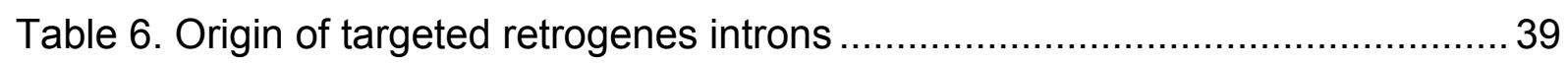




\section{LIST OF ABBREVIATIONS}

$\begin{array}{ll}\text { cDNA } & \text { complementary DNA } \\ \text { CDS } & \text { Coding DNA Sequence } \\ \text { Chr. } & \text { Chromosome } \\ \text { DNA } & \text { Deoxyribonucleic Acid } \\ \text { gcRMA } & \text { guanine cytosine Robust Multi-array Average } \\ \text { gDNA } & \text { genomic DNA } \\ \text { GO } & \text { Gene Ontology } \\ \text { GW } & \text { Genome Wide } \\ \text { H3K9me2 } & \text { Histone H3 lysine 9 di-methylation } \\ \text { H3K27me3 } & \text { Histone H3 lysine 27 tri-methylation } \\ \text { kbp } & \text { kilo base pair } \\ \text { LINE } & \text { Long Interspersed Nuclear Element } \\ \text { MWW } & \text { Mann-Whitney-Wilcoxon } \\ \text { MYA } & \text { Million Year Ago } \\ \text { PCR } & \text { Polymerase Chain Reaction } \\ \text { RNA } & \text { Ribonucleic Acid } \\ \text { RPKM } & \text { Reads Per Kilobase per Million mapped reads } \\ \text { TE } & \text { Transposable Element } \\ \text { TSS } & \text { Transcription Start Site } \\ \text { TTS } & \text { Transcription Termination Site }\end{array}$




\section{ACKNOWLEDGEMENT}

I would like to thank Dr. Ales Pecinka for supervision during my work in his group at Max Planck Institute for Plant breeding research. I'm very grateful for Professor Dr. Maarten Koornneef, not only for allowing me to work in his department; but also for being the caring and understanding director he is. I'm also thankful to my supervisors at University of Göttingen, Professor Dr. Christiane Gatz and Professor Dr. Stefan Jakobs for their support and supervision.

Many thanks are to current and former group members of Pecinka and Jiménez-Gómez groups: Dr. Andreas Finke, Björn Pietzenuk, Ganga Jeena, Dr. Inga Schmalenbach, Dr. Marcel von Reth, Mariana Diaz, Mohamed El Hadidi, Navratan Bagwan, Dr. Niels Müller, Dr. Thomas Piofczyk, and Dr. Tripta Jhang for the provided working environment. Special thanks are to Arunkumar Srinivasan for the useful discussions and brain storming, as well as for the bioinformatics consultation. I also thank Barbara Eilts, Regina Gentges and Petra Pecinkova for taking care of plants and technical assistance. Special appreciation to the support and organization provided by the department secretary Birgit Thron. Many thanks are to administration and faculty members of the faculty of Agriculture, Cairo University, with special thanks to members of the department of Genetics, Cairo University for their continuous support.

My success in my studies has been positively influenced by the continuous encouragement and support of Dr. Ahmed Ashoub and his family, to whom I'm deeply grateful. Additionally, I'm thankful to my former teachers and friends who had positive influence on my scientific and personal life, particularly Adham Saleh, Mahmoud Negm, Mohamed Aman, Mohamed Soliman and my dear beloved brother Gaser Abdelsamad.

My love is to my family, specially my parents, whose love and care supported and shaped my life and progress, and to whom I'm totally indebted. My love is also to my wife and mother-in-law who cheered my life. Finally, and before all, all praise is to the God the all-wise, the all-knowing the creator of this wonderful world. 


\section{DECLARATION}

Herewith I declare that I prepared the PhD thesis entitled "Evolution and epigenetic regulation of RNA-mediated duplicated genes in Arabidopsis" on my own and with no other sources and aids than quoted. I also affirm that the facilities of Max Planck Institute for Plant Breeding Research were the only facilities utilized during the practical work.

Göttingen, Germany on 28.05.2015

Signature:

Ahmed M. Abdelsamad 


\section{INTRODUCTION}

Gene duplications are an important factor in genome evolution allowing for functional diversification of genes (Flagel and Wendel, 2009; Innan and Kondrashov, 2010). Duplicated genes are generated by several DNA- and RNA-based mechanisms (Innan and Kondrashov, 2010; Sakai et al., 2011). Whole genome DNAbased duplication (WGD) by polyploidization has occurred in the evolutionary history of all land plants and many animals (De Smet et al., 2013; Dehal and Boore, 2005). Since WGD amplifies the entire genome, it seems to be a solution towards major evolutionary and/or ecological challenges (Comai, 2005; Fawcett et al., 2009). However, WGDs do not alter protein stoichiometry in most cases and therefore, they may be relatively ineffective in situations when an increased amount of a single or few specific proteins is required. In such situation, local DNA and RNA duplication mechanisms may be a better fitting solution. Local DNA duplications amplify individual genes or short chromosomal regions, presumably by an unequal crossing over mechanism (Zhang, 2003). In RNA-based duplication (retroposition), the mature mRNA of a protein-coding gene is reverse transcribed and integrated at ectopic position in the genome using retroviral or retrotransposon machinery (Kaessmann et al., 2009). Therefore, retroposition has a high potential to generate evolutionary innovations, e.g. by expressing genes in a new developmental context, generating chimeric genes with new functional domain combinations or inter-specific horizontal gene transfer (Sakai et al., 2011; Wang et al., 2006; Yoshida et al., 2010).

Gene copies generated through retroposition are called retrogenes, and are distinguished from retrotransposons. Their precursor mRNA molecules are transcribed from non-transposable element protein coding genes (parental genes) that are involved in diverse biological processes (Kaessmann et al., 2009). Consequently, retrogenes are also involved in diverse biological processes and human diseases, such as cancer (Cooke et al., 2014; Hirotsune et al., 2003). Relatively few studies have conducted genome-wide search for retrogenes in plants (Zhang et al., 2005; Wang et al., 2006; Zhu et al., 2009; Sakai et al., 2011). They identified retrogenes to be at most $0.38 \%$ of protein coding genes, except for a study in rice (Oryza sativa) where low stringency selection criteria were applied (Wang et al., 2006). In humans, $19.1 \%$ of all genes were identified as retrogene copies; 
however, $82 \%$ of those copies contain premature stop codons. Therefore, only $3.4 \%$ of all human genes are putatively functional retrogene copies producing functional proteins (Marques et al., 2005; Pennisi, 2012). In rice, transcription was observed for two-thirds of retrogenes, indirectly suggesting that there may be higher proportion of functional retrogenes in plants (Sakai et al., 2011).

Since retroposition duplicates only transcribed regions, it is expected to cause the loss of promoter sequences. This may represent a major bottleneck to retrogene evolutionary success. Recent studies in human and rice suggested retroposition including parental gene promoter (Okamura and Nakai, 2008; Sakai et al., 2011). Additionally, there are multiple possible mechanisms of retrogene promoter acquisition that have been demonstrated in individual examples (Kaessmann et al., 2009). Nevertheless, it is often not clear how frequent those mechanisms are at the genome-wide scale.

Retrogenes expression may be suppressed by epigenetic mechanisms that target transposons and repetitive elements (Vaucheret and Fagard, 2001). Retrogenes are generated by retrotransposon reverse transcriptases and represent duplicated copies; therefore they may become targets of epigenetic transcriptional gene silencing (TGS) by repressive chromatin marks. Chromatin is an indispensable component that provides regulatory and protective function to genetic information (reviewed in e.g. (Li et al., 2007). Transcribed protein coding genes are associated with permissive chromatin marks. In contrast, transcriptionally repressed genes and repetitive elements are typically labeled by histone $\mathrm{H} 3$ lysine 27 tri-methylation (H3K27me3), histone H3 lysine 9 di-methylation (H3K9me2) and/or high density DNA methylation in all cytosine sequence contexts in plants (Liu et al., 2010; Roudier et al., 2011; Stroud et al., 2013). While H3K27me3 ensures tissue specific developmental transcription (Lafos et al., 2011), the role of H3K9me2 and promoter DNA methylation is to minimize activities of all kinds of repetitive elements, which frequently includes retrotransposons (Ibarra et al., 2012; Mosher et al., 2009; Slotkin et al., 2009). The association of retrogenes with specific chromatin states has been proposed (Boutanaev et al., 2002; Marques et al., 2005), but only few animal and no plant retrogenes have been characterized as to their chromatin states so far (Monk et al., 2011; Pei et al., 2012).

In flies and mammals, many retrogenes show specific transcription in male germ cells (Bai et al., 2008; Marques et al., 2005; Vinckenbosch et al., 2006). This 
pattern is intriguing and several explanatory models have been proposed (reviewed in Kaessmann et al., 2009; Kaessmann, 2010). First, it could originate from various chromatin modifications affecting chromosomes and leading to hyper-transcription in meiotic and post-meiotic spermatogenic cells. As a consequence of this global chromatin reorganization induced transcription, some of the testis-transcribed retrogenes could also evolve testis-specific gene functions. The second, not mutually exclusive, hypothesis postulates that retrogenes amplify in the germline tissues and insert preferentially into actively transcribed (open) chromatin. This creates a selfreinforcing loop where the retrogenes insert nearby or into germline transcribed genes and consequently would be also germ-line transcribed. The latter hypothesis is partially supported by observations in Drosophila (Bai et al., 2008), but the tissuespecificity in transcription of plant retrogenes has not been studied.

This study aims to investigate plant retrogenes and their parental genes concerning their abundance, distribution in the genome, expression pattern, relation to transposable elements, epigenetic regulation, emergence rate and evolution. We generated deep sequencing transcriptome data, and used the comprehensive genome and transcriptome resources for the closely related Arabidopsis thaliana and Arabidopsis lyrata to investigate these open questions. We had manually identified retrogenes in $A$. thaliana genome that were not reported in previous screens (Zhang et al., 2005; Zhu et al., 2009). Therefore, we developed a novel bioinformatic retrogene annotation tool (RAT) to screen both genomes, and initially identified 251 A. thaliana retrogenes, 216 of which are novel. We used this set together with the retrogenes found previously (Appendix A) to analyze retrogene and parent-specific features. We show that parents are usually ubiquitously transcribed while retrogenes are mainly low and stage-specific transcribed. Most $A$. thaliana retrogenes acquired novel cis-regulatory elements at their integration sites. Importantly, throughout plant development, retrogenes show peak of transcription in pollen. This pattern can also be observed for many lowly transcribed genes genome-wide and resembles retrogene transcription in testis of animals. We found that pollen-specific activation of A. thaliana retrogenes is associated with global transcriptional reprograming (Abdelsamad and Pecinka, 2014).

In the second part of this study, we used our enhanced version (v2) of $A$. lyrata genome annotation and identified $168 \mathrm{~A}$. lyrata retrogenes representing the first of identified retrogenes in $A$. lyrata. We show that Arabidopsis retrogenes 
emerge in the genome at least ten-times faster than previously calculated (Zhang et al., 2005). Most of the identified retrogenes are transcribed (putatively functional); and targeted by $21 \mathrm{nt}$ sRNA molecules, unlike retrotransposons that share the same duplication machinery. Retrogenes tend to acquire introns, which significantly extend retrogene mRNA half-life. We show that not all nascent retrogenes integrate randomly in the genome. Some retrogenes specifically replace their parental genes in a process called retrogene targeting. We developed targeted retrogene annotation tool (TRAT), as an additional tool, to screen the genomes for these cases. Based on current literature, we believe that we report the first natural in planta retrogene targeting events. 


\section{RESULTS}

We aimed to study evolution, expression, epigenetic regulation and abundance of retrogenes and their parental genes in plant genomes. We had manually identified retrogenes that were not reported in the previous annotations of retrogenes in $A$. thaliana (Zhang et al., 2005; Zhu et al., 2009). Therefore, we developed a novel bioinformatic retrogene annotation tool to conduct a genome-wide search for retrogene-specific features in $A$. thaliana genome (TAIR10). The identified retrogenes were then studied extensively.

\subsection{Annotation of $A$. thaliana retrogenes by a novel retrogene annotation tool (RAT)}

We developed a novel bioinformatic Retrogene Annotation Tool (RAT) to conduct a genome-wide screen for retrogenes (Figure 1A). In total 251 retroposition events satisfying stringent quality criteria were annotated in $A$. thaliana genome (Appendix A). Among retrogenes identified in our list, 36 were shared with two previous genome-wide retrogene screens (Zhang et al., 2005; Zhu et al., 2009) and 216 were novel (Figure 1B). The total number of retrogenes identified in all three studies is 309 (291 were considered for downstream analyses; see Appendix A) and corresponds to approximately $1 \%$ of $A$. thaliana protein coding genes and pseudogenes ( $n=27,416$ and 924 , respectively).

Generally, retrogenes are intron-less copies of intron-containing paralogous genes. They integrate randomly in the genome; and potentially have downstream poly(A)-tails. The RAT screened for theses retrogene-specific characters. The principal steps in retrogene identification are given in (Figure 1A). First, the paralogy groups between sets of intron-less and intron-containing protein coding genes according to TAIR10 were established using protein homologies in InParanoid Version 4.1 with default parameters (Remm et al., 2001). When the paralogy group had multiple intron-containing 'inparalogs' with $\geq 2$ different introns, they were also considered for downstream analysis. Similarly, paralogy groups between pseudogenes and intron-containing protein coding genes were identified as the best reciprocal BLAST hits using cDNA sequences (Altschul et al., 1990; Swarbreck et al., 
2008). Accepted retrogene-parent candidate pairs had a minimum homology score $10^{-10}$ and a minimum difference in intron number of two introns. A single intron difference was only accepted if a poly(A)-tail was detected within 150 or 250 bp downstream of the stop codon of the retrogene candidate with or without annotated $3^{\prime}$ UTR, respectively. Poly (A)-tail was defined as a stretch of consecutive adenines with minimum length of 15 adenine nucleotides, allowing a single mismatch. We determined Poly $(A)$-tail minimum length as the shortest non-random stretch of $(A)$ nucleotides present in $A$. thaliana genome (materials and methods; Figure 18).

A

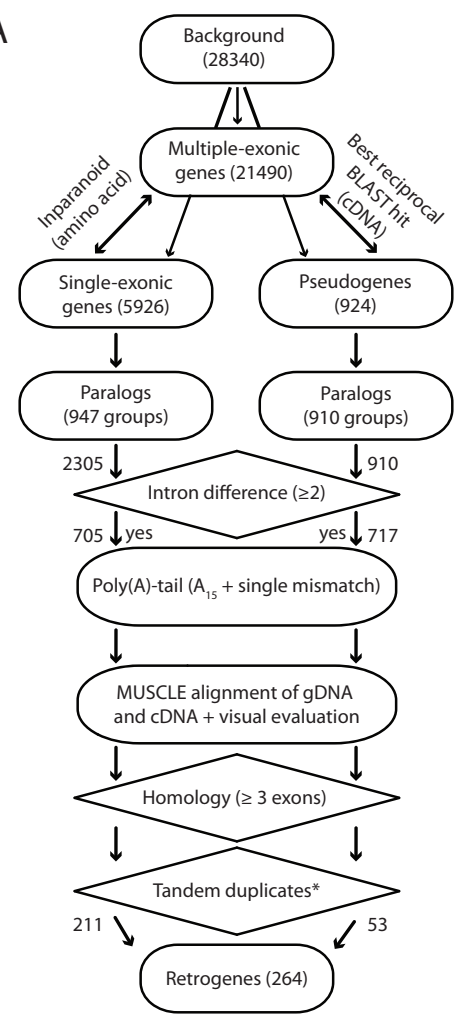

B

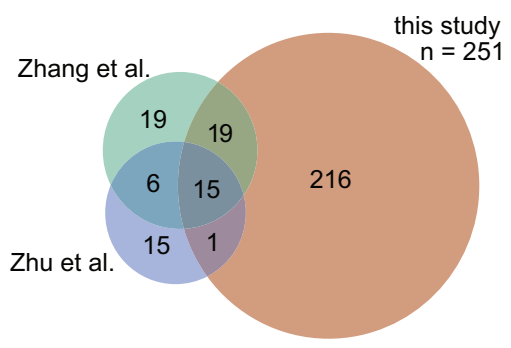

C

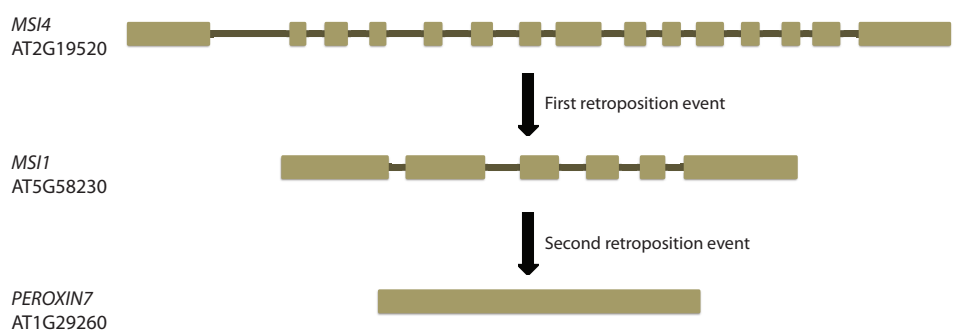

Figure 1. Annotation of $A$. thaliana retrogenes using the RAT

(A) Schematic representation of the retrogene annotation tool. (B) Venn diagram indicating the numbers of retrogenes identified in three $A$. thaliana genome-wide searches (Abdelsamad and Pecinka, 2014; Zhang et al., 2005; Zhu et al., 2009). The venn does not include disputable retrogenes from the two previous studies. (C) Example of repeated retroposition in A. thaliana; the MSI4 - MSI1 - PEROXIN 7 retroposition series.

Since the absence of introns can also be due to a loss of splicing signals (intron retention), homology of exonic and intronic sequence was visually validated following gDNA and cDNA sequence alignment (Edgar, 2004). A retrogene was 
accepted when a minimum of three consecutive homologous exons, spanning two lost introns, were observed. If multiple parents were predicted for a retrogene, we accepted the candidate with the highest pairwise alignment score in multiple (cDNA) sequence alignment (Deng et al., 2010; Larkin et al., 2007). When a candidate retrogene overlaps with a list of DNA-based gene duplications (Blanc and Wolfe, 2004), it was excluded. The protocol was executed with customized bioperl and awk scripts (Stajich et al., 2002).

Hence, the RAT identified 251 A. thaliana retroposition events; that were used for downstream analysis together with previously identified retrogens.

\subsection{A. thaliana retrogenes are capable of repeated retroposition and occur in gene-rich genomic regions}

The RAT tool combines multiple retrogene searches within intron-less and intronized genes; thus, it allows searching for potential secondary retropositions of retrogene transcripts. This revealed 12 retrogenes that served as templates for another round of retroposition (Figure $1 \mathrm{C}$ and Table 1). In these cases, the primary parent gave rise to the primary retrogene, whose mRNA served as the precursor for the secondary retrogene. The model where the primary parent gives rise directly to the secondary retrogene was not supported by the order of protein homologies, and that suggests retroposition of the retrogene transcript. Hence, $4.3 \%$ of $A$. thaliana retrogenes underwent repeated retroposition without losing their protein coding potential. In addition, we identified multi-retrogene parents. In total, 22 parents gave rise to 54 retrocopies $(17 \times 2 ; 3 \times 3 ; 1 \times 4 ; 1 \times 7)$ and a maximum of seven retrocopies derived from a single parent (Appendix $A$ ). The observed frequency of multiple retropositions from the same gene is significantly higher than expected at random (Mann-Whitney-Wilcoxon (MWW) test, $P<2.2 \times 10^{-16}$ ) strongly arguing that the selection of parental mRNA is not random in at least some cases.

The machinery that transposes retrogenes and retrotransposons often integrate the later at hereochromatic regions (Tsukahara et al., 2012). To explore whether retroposition of retrogenes occurs at specific genomic regions, we plotted densities of all protein coding genes, transposable elements (TEs), parents and retrogenes over the five $A$. thaliana chromosomes (Figure 2A). In agreement with 
published data (Arabidopsis Genome Initiative, 2000), TEs were enriched in pericentromeric regions and depleted from chromosome arms, while protein-coding genes showed the opposite pattern. Both retrogenes and parents had a profile similar to that of protein coding genes, showing that they occur preferentially in gene-rich genomic regions (Figure $2 \mathrm{~A}$ ).

Table 1. Repeated retroposition events in $A$. thaliana

\begin{tabular}{|c|c|c|c|c|c|}
\hline \multicolumn{2}{|c|}{ Parent } & \multicolumn{2}{|c|}{ Retroposition 1} & \multicolumn{2}{|c|}{ Retroposition 2} \\
\hline Gene ID & Introns & Gene ID & Introns & Gene ID & Introns \\
\hline AT1G08320 & 12 & AT1G77920 & 8 & AT1G58330 & 0 \\
\hline AT1G58520 & 16 & AT1G32090 & 10 & AT1G30360 & 5 \\
\hline AT2G19520 & 14 & AT5G58230 & 5 & AT1G29260 & 0 \\
\hline AT2G28830 & 6 & AT3G46510 & 3 & AT1G29340 & 0 \\
\hline AT3G09100 & 16 & AT5G01290 & 14 & AT5G28210 & 0 \\
\hline AT3G09810 & 6 & AT4G35260 & 3 & AT1G32480 & 0 \\
\hline AT3G24430 & 13 & AT4G19540 & 7 & AT5G50960 & 2 \\
\hline AT4G34480 & 5 & AT5G24318 & 3 & AT3G55430 & 1 \\
\hline AT4G40040 & 4 & AT5G10980 & 2 & AT5G10400 & 0 \\
\hline AT5G28340 & 5 & AT3G60960 & 2 & AT3G60980 & 0 \\
\hline AT5G56890 & 13 & AT1G70460 & 7 & AT3G55950 & 0 \\
\hline AT5G67320 & 13 & AT2G26060 & 9 & AT1G24530 & 0 \\
\hline
\end{tabular}

We showed that retrogenes integrate preferentially in chromosome arms; however, they may still integrate nearby local TEs. To test for association of retrogenes and/or parents with TEs at local scale, we estimated the frequency of all genes with TEs in $1 \mathrm{kbp}$ intervals up- and down-stream of gene transcription start and termination sites (TSS and TTS, respectively). On average, there were fewer TEs upstream than downstream of genes. The frequency of TEs in TSS-upstream regions for all protein-coding genes and retrogenes (17\% and $22 \%$, respectively) was not significantly different (Figure $2 \mathrm{~B}$ ). In contrast, parental genes with TEs in the first two kbp upstream of the TSS were scarce relative to the whole genome (chi-square test, $P<0.05)$. Similarly, $25 \%$ of all genes and retrogenes contained TEs in the first two kbp of the TTS-downstream region, while it was only $17 \%$ for parents (chi-square test: $P<0.05$ in the first kbp). This shows that retrogenes are not enriched for closelying TEs compared to the genomic average, but parents are depleted of TEs in both up- and down-stream intergenic regions. 
Hence, the $A$. thaliana genome contains at least 291 retrogenes located predominantly in gene-rich chromosomal regions. About $10 \%$ of the parents gave rise to multiple retrogenes and approximately $4.3 \%$ of the retrogenes underwent a second retroposition.
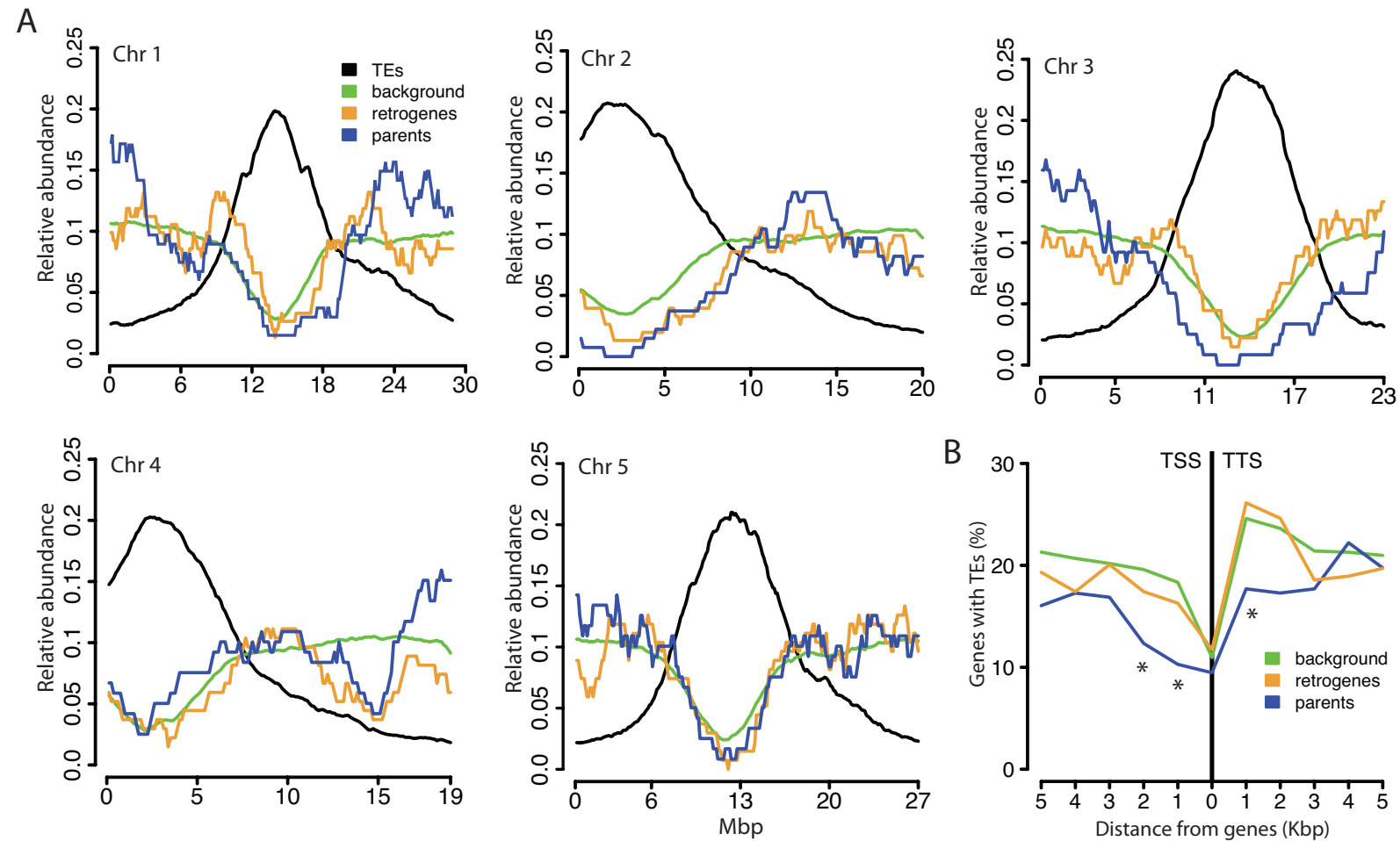

Figure 2. Genomic features of $A$. thaliana retrogenes

(A) Relative abundance (y-axis) of transposable elements (TEs, black), all genes and pseudogenes (background, green), retrogenes (red) and parents (blue) over the five $A$. thaliana chromosomes ( $x$-axis). (B) Percentage of genes containing TEs (y-axis) in $1 \mathrm{kbp}$ intervals from the gene transcription start and termination sites (TSS and TTS, respectively) for all protein coding genes (background, green), retrogenes (red) and parents (blue). Significant differences $(P<0.05)$ in chi-square test relative to background are indicated by asterisk. 
A
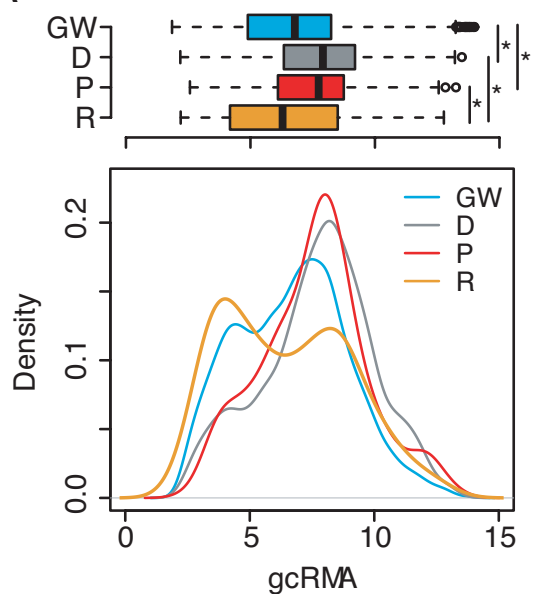

C

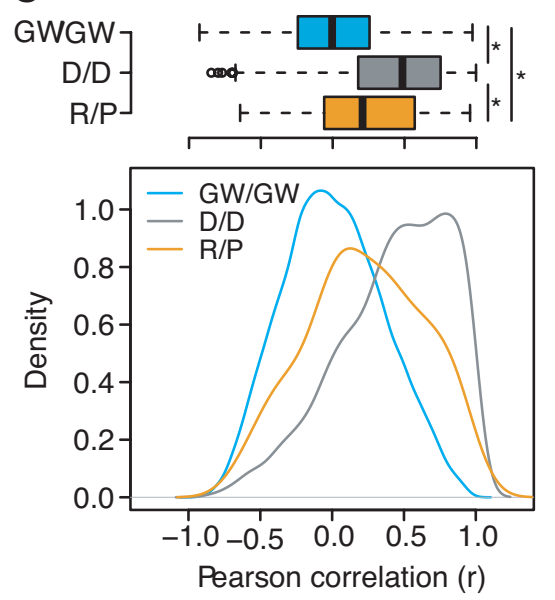

$\mathrm{B}$

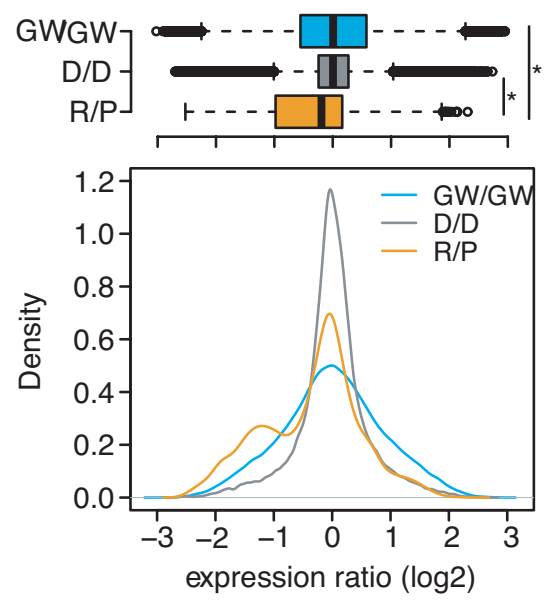

D
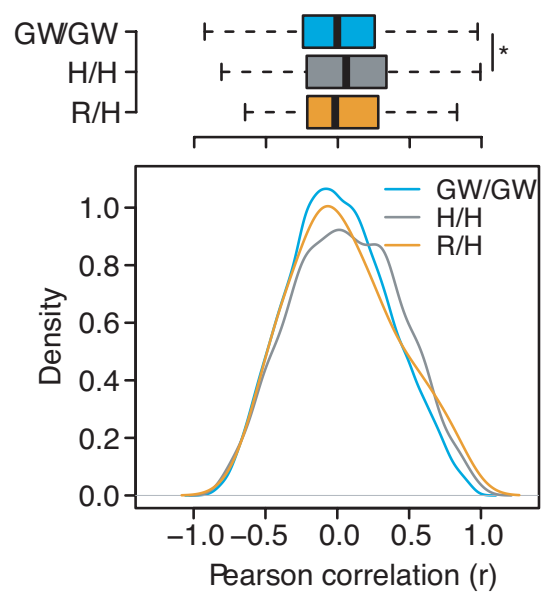

$E$

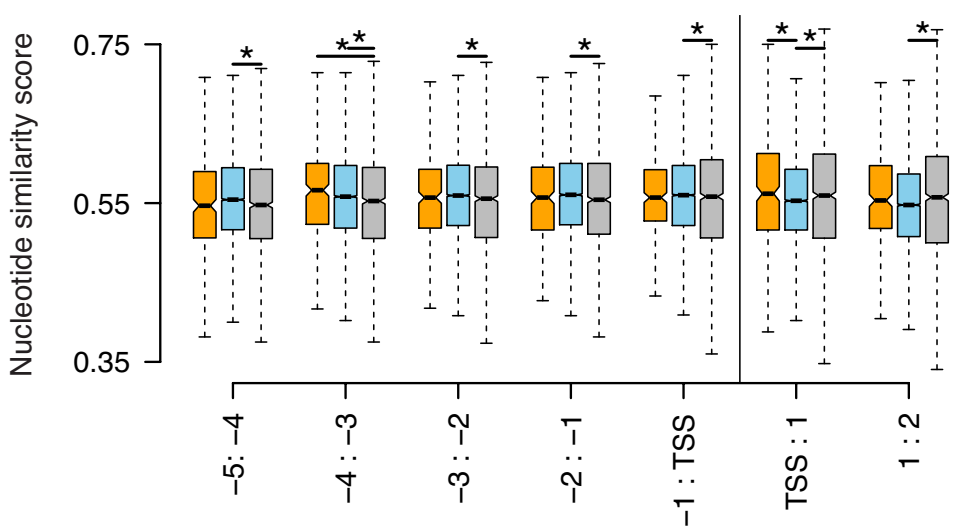

(x100) nucleotide pins of promoter sequence

\section{Figure 3. Retrogenes are driven by novel promoters}

(A) Box and density plots of $\log _{2}$ robust microarray averaging (gcRMA) values for genomewide genes (GW), DNA duplicated genes (D), parents $(P)$ and retrogenes $(R)$ over the $49 \mathrm{~A}$. thaliana developmental stages. (B) $\log _{2}$ transcription ratios of the random genome-wide gene pairs (GW/GW), DNA duplicated pairs (D/D) and retrogene/parent pairs (R/P). (C, D) Pearson correlation of gene co-transcription between random genome-wide gene pairs (GW/GW), DNA duplicated pairs (D/D), retrogene-parent pairs (R/P), genome-wide head-to- 
head oriented genes $(H / H)$ and retrogene-head-to-head oriented neighboring genes $(R / H)$ in 49 developmental stages. (E) Box plots of nucleotide similarity score for (x100) nucleotidelong pins of promoter sequences. Nucleotide similarity scores for retrogene-parent promoters (orange) are not significantly different from for random gene pairs (sky blue), but usually less than for DNA duplicated gene pairs (grey). Non-significant $(P \geq 0.05)$ relationships are not shown.

\subsection{Retrogenes are derived from highly transcribed parental genes and are transcribed preferentially by novel promoters}

The cDNA origin of retrogenes implies their retroposition without their regulatory sequences (promoters). However, the majority of them retains intact open reading frames (ORFs) and is transcribed. We took advantage of the comprehensive retrogene list assembled in this study (Appendix A) (Abdelsamad and Pecinka, 2014) and explored the patterns of retrogene transcription in $A$. thaliana. The mRNA accumulation was analyzed using microarray data from the $49 A$. thaliana developmental stages assembled by the AtGenExpress consortium (Schmid et al., 2005) and validated for selected tissues by RNA-sequencing (Loraine et al., 2013). In total, 209 retrogenes and 245 parents are present on the ATH1 cDNA microarray. To compare the effects of RNA- and DNA-based duplications, we also analyzed the set of 3,088 A. thaliana DNA duplicated genes (Blanc and Wolfe, 2004). Plotting the mean $\log _{2}$ Robust Multi-array Averaging (gcRMA; (Irizarry et al., 2003) values of all ATH1 probesets $(n=22,746)$ revealed a double-peak distribution with the left peak representing genes with poor mRNA levels and/or background signals (Figure 3A). The gcRMA values of some retrogenes and parents overlapped with this region and suggested that some of the candidates may not be transcribed in any of the 49 stages. Therefore, we kept only the genes with gcRMA values of 5 or higher in at least one developmental stage (transcribed genes). In total, 89.4\% ( $n=20,398$ ) of all genes, $85.2 \%(n=178)$ of retrogenes, $94.7 \%(n=232)$ of parents and $99.3 \%(n=$ 3067) of DNA duplicated genes passed these criteria (Figure 3A). This shows that the majority of $A$. thaliana retrogenes are transcribed in at least some developmental stages and their mean gcRMA values did not differ significantly from the genomewide gene set (MWW test, $P=0.48$; Figure $3 \mathrm{~A}$ ). The parents were significantly enriched for highly transcribed genes relative to both retrogenes and the wholegenome set (MWW test, $P=7.64 \times 10^{-06}$ and $P=1.86 \times 10^{-11}$, respectively; Figure 
3A). Similarly, DNA duplicated genes were strongly transcribed and therefore similar to parents, but strongly different from retrogenes (MWW test, $P=0.16$ and $P=1.56 \times$ $10^{-10}$, respectively).

To reveal the transcription relationships between individual retrogene-parent pairs, we compared their developmental stage-specific gcRMA ratios with the transcription of 5,000 randomly selected gene-pairs and the 1,527 DNA duplicated gene-pairs (Figure 3B). Transcript accumulation ratios of random pairs and DNA duplicated genes represented a broad and narrow range of normally distributed values (MWW test, $P=0.85$ ). Although many retrogenes have a comparable degree of transcription relative to their parents, there is a specific group of two-to-three-fold less transcribed retrogenes making retrogene-parent pairs significantly different from both the random gene set and DNA duplicated genes (MWW test, both comparisons $P<2.2 \times 10^{-16}$; Figure 3B). Inspecting the gcRMA values over individual developmental stages for the low-transcribed group revealed that these retrogenes were transcribed above the threshold ( $g \mathrm{CRMA} \geq 5$ ) in only one or few tissues while their parents frequently showed ubiquitous transcription.

A recent study in rice suggested frequent co-transcription between retrogenes and parents in plants (Sakai et al., 2011). Our retrogene identification criteria and the nature of $A$. thaliana retrogenes (e.g. an absence of retrogenes residing in the introns of other genes) allowed testing three possible mechanisms of retrogene cisregulatory element origin: 1) carry-over of parental promoters, 2) the use of bidirectional promoters at integration sites, and 3 ) an acquisition of novel cis-regulatory elements. First, we tested whether the $A$. thaliana retrogenes inherit the parental transcription pattern. We calculated co- transcription of retrogene-parent pairs as Pearson product-moment correlation coefficients $(r)$ across the 49 developmental samples of the AtGenExpress dataset. Indeed, co-transcription in the set of retrogene-parent pairs $(n=179)$ was significantly higher than in the 20,000 randomly selected gene pairs (MWW test, $P=2.30 \times 10^{-6}$; Figure $3 \mathrm{C}$ ). We calculated the frequencies of genes per $0.1 r$ correlation bins for retrogenes and genome background and used this to calculate the number of highly co-transcribed retrogene-parent pairs. In total, $25 \%$ of the retrogene-parent pairs (26 out of 102) were correlated more than random gene pairs. However, the co-transcription of DNA duplicated gene pairs, calculated in the same way, was more prominent (MWW test, 
$P<2.2 \times 10^{-16}$; Figure $3 \mathrm{C}$ ) and $45.6 \%$ of them surpassed the random-pairs background.

Second, we tested the possibility for retrogene transcription by bi-directional promoters of head-to-head ("head") oriented neighboring genes. The Pearson correlations of random transcribed gene-pairs $(n=20,000)$ and the genome-wide set of transcribed "head" oriented genes $(n=2,087)$ revealed an infrequent but consistent co- transcription between head-oriented gene pairs (MWW test, $P=2.705$ $\times 10^{-10}$; Figure 3D). This shows that sharing bi-directional cis-elements is not common in $A$. thaliana. Retrogene-head oriented neighbor pairs ( $n=63$ ) displayed an intermediate pattern that was not significantly different from either genome-wide or head oriented genes (MWW test, both $P=0.60$; Figure 3D). Only $2.5 \%$ of head oriented retrogenes had higher correlation than random pairs, illustrating negligible effect of promoter sharing (Figure 3D). Consequently, retrogenes seemed to acquire novel cis-regulatory sequences at their integration sites. The low nucleotide similarity scores between retrogenes and parental gene promoters supported this hypothesis; that were not significantly different from scores for random gene pairs (GW) but significantly less than for DNA duplicated gene pairs (Figure 3E).

Hence, retrogenes show low transcription, while their parents show high and ubiquitous transcription. The transcription of most of the retrogene-parent pairs is not correlated, due to acquisition of novel regulatory elements at retrogene integration sites.

\subsection{A. thaliana retrogenes are transcribed in male gametes}

In insects and animals, retrogenes show preferential transcription in male germ cells (Kaessmann, 2010). To analyze developmental regulation of $A$. thaliana retrogene transcription, we plotted the mean gcRMA values of genome-wide, parent and retrogene sets for each of the 49 analyzed developmental stages (Figure 4A). The average mRNA level of parents was higher than that of retrogenes and the genome-wide gene set in all stages. The mean transcription per group was relatively constant, except for pollen where there was a dip in transcription in the parents and the genome-wide set that was contrasted with a peak of retrogene transcription (Figure 4A). To identify relationships between developmental stages and retrogenes, 
we hierarchically clustered both groups and expressed the result as a heat-map of the retrogene transcription z-scores (Figure 4B). This separated stamen and pollen from the rest of the tissues. The highest frequency of retrogenes with positive zscores $(>0)$ was then found in pollen and seeds $(62 \%$ and $63 \%$, respectively; Figure 4C). However, with more stringent criteria (z-scores $>1$ and $>3$ ), the pollen peak became more prominent relative to other tissues and corresponded to $50 \%$ and $30 \%$ of retrogenes, respectively (Figure 4C). This shows that many retrogenes reach their transcription maxima in pollen. The pollen-specific transcription pattern has been confirmed by analysis of individual cases (Figure 4D, Figure 5A).

However, plotting the transcription quantiles (Appendix C) of retrogene $\log _{2}$ gcRMA revealed that not all retrogenes followed this simple trend; and the retrogenes with a negative z-score (pollen down-regulated) usually derived from the group of developmentally highly transcribed genes (Figure 4E, bottom). Remarkably, this distribution also held true for the genome-wide gene set (Figure 4D, top). The parents and the DNA duplicated genes showed more prominent down-regulation of the highly transcribed genes (quantile 4) and less obvious up-regulation of lowly transcribed genes (quantile 1), while TEs showed up-regulation for all quantiles (Figure 5B). Hence, we found a pollen specific activation of retrogenes that is a part of the global pollen-specific transcriptional reprogramming. 
A
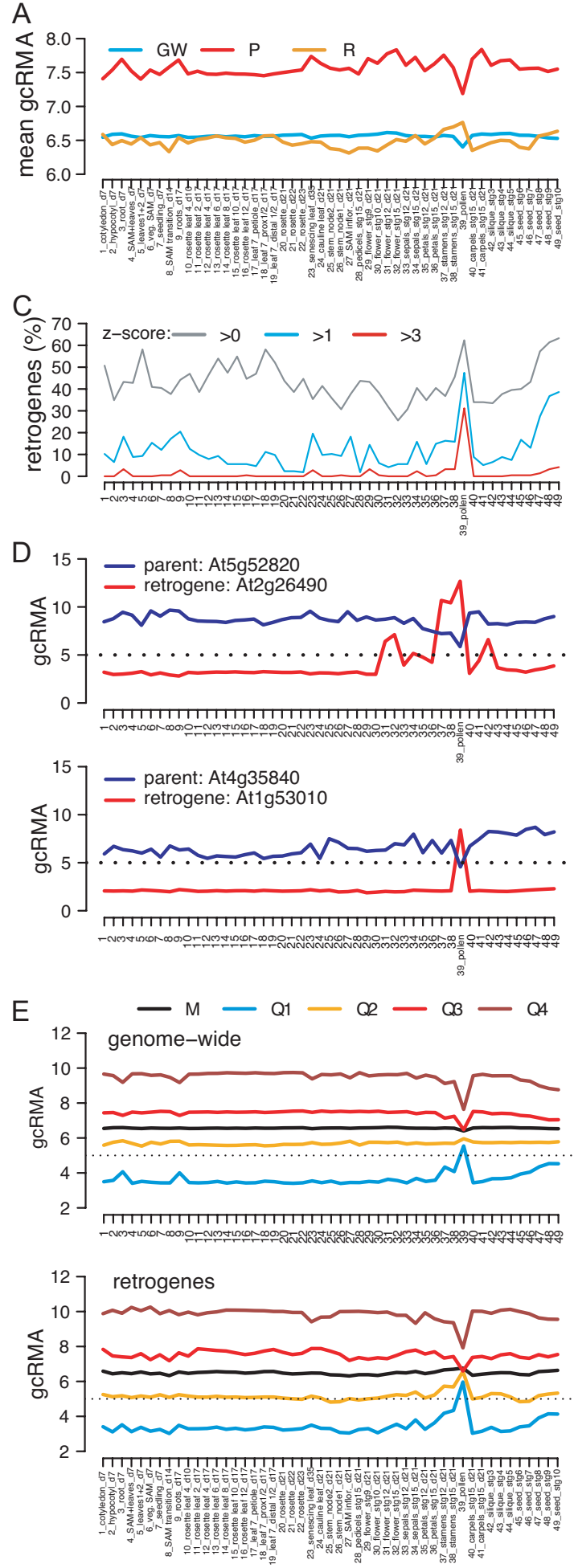

B

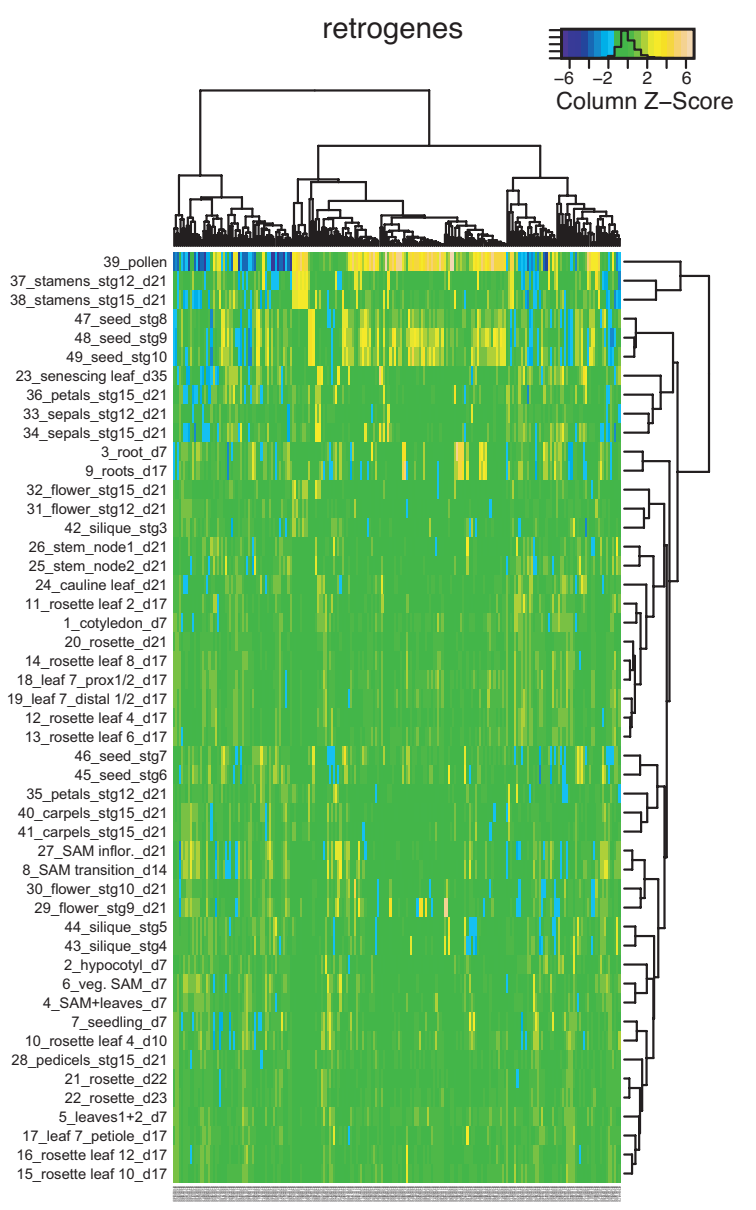

F
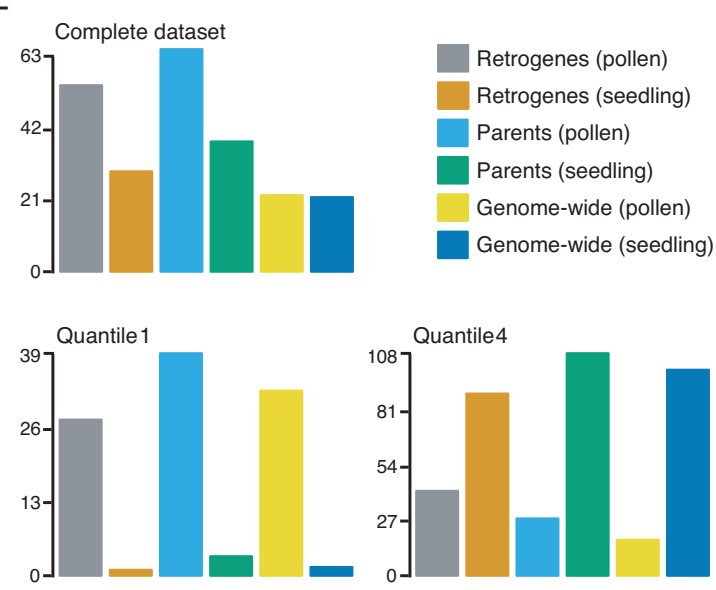

\section{Figure 4. Retrogenes are transcriptionally up-regulated in pollen}

(A) The mean $\log _{2}$ robust microarray averaging (gcRMA) values for genome-wide genes $(\mathrm{GW})$, parents $(\mathrm{P})$ and retrogenes $(\mathrm{R})$ at each of the $49 \mathrm{~A}$. thaliana developmental stages. (B) Hierarchically clustered heat map of retrogene z-scores ( $y$-axis) and developmental stages ( $x$-axis). (C) The frequency of retrogenes with row z-scores in (B) $>0,>1$ and $>3$ in individual developmental stages. (D) Examples of retrogenes and parents showing tissuespecific and ubiquitous transcription, respectively, with major transcription changes in pollen (stage 39). (E) Developmental gcRMA values for genome-wide set of genes and retrogenes. 
Transcription is shown for mean $(\mathrm{M})$ and transcription quantiles: low-transcribed/quantile 1 (Q1), mid-low-transcribed/quantile 2 (Q2), mid-high-transcribed/quantile 3 (Q3) and hightranscribed/quantile 4 (Q4). (F) Mean RNA-sequencing RPKM values ( $y$-axis) for all genes (Genome-wide), parents and retrogenes in vegetative rosettes and pollen as complete datasets, quantile 1 (lowly transcribed genes) and quantile 4 (highly transcribed genes).

Pollen development includes several stages (Honys and Twell, 2003). To find out whether retrogenes are transcribed in specific pollen developmental stages, we compared their transcription in the three final developmental stages; unicellular microspores, bicellular pollen, tricellular pollen and two highly correlated $(r=0.92)$ samples of mature pollen grains (Honys and Twell, 2004; Schmid et al., 2005). This revealed continuous increase of mean retrogene transcription throughout pollen development that contrasted with down-regulation of parental genes in tri-cellular pollen and mature pollen grains (Figure $5 \mathrm{C}$ ). There are two distinct cell types in mature pollen: vegetative and sperm cells. Thus, we investigated whether there is enrichment for retrogene transcripts in vegetative and sperm cells (Honys and Twell, 2003). We used TEs as the control for vegetative cell specific transcription based on the recently proposed model (Slotkin et al., 2009). Although we observed strong TE up-regulation in pollen relative to leaves (MWW test, $P<2.2 \times 10^{-16}$ ), there was a significantly higher amount of TE transcripts in sperm cells relative to the entire pollen (MWW test, $P=0.013$; Figure $5 \mathrm{C}$ ). This indicates that there is a higher amount of TE transcripts in both pollen cell types. The parents were significantly more transcribed in sperm cells relative to seedlings (MWW test, $P=0.001$ ) and were underrepresented for the low transcribed genes in this tissue relative to entire pollen (Figure 5C). Therefore, retrogene parents are transcribed preferentially in sperm cells. The median of retrogene transcription was higher than that of TEs and increased in both pollen samples relative to seedlings, but only the entire pollen differed significantly (MWW test, $P=0.008$; Figure $4 \mathrm{E}$ ). In combination with pollen developmental series data, this shows that retrogenes are transcribed in both pollen cell types.

In order to validate our results by independent experiment, we tested whether our findings hold true in datasets generated by RNA-sequencing. Gene transcription in mature pollen grains was compared with that in seedling tissues (Loraine et al., 2013). Plotting the mean RPKM (reads per one kilobase per one million reads) values for entire set, quantile 1 (lowest transcribed) and quantile 4 (highest transcribed) of all genes, retrogenes and parents confirmed microarray data (Figures 
4A, E, F; Figure 5B). The only exception was higher transcription of parents in pollen relative to seedlings in RNA-sequencing (mean and quantile 1 samples; absent in quantile 4 sample) while this was opposite in microarrays (Figure 4A,F). This difference can be attributed to higher sensitivity of RNA-sequencing technology to quantify transcripts from low transcribed genes (Mooney et al., 2013; Zhao et al., 2014). This partially applies also to retrogenes as the up-regulation in pollen versus seedling is more pronounced in RNA-seq compared to microarrays (Figure 4F). From this we conclude that retrogene activation starts prior to pollen maturation and later occurs in both terminal pollen cell types (vegetative and sperm cells).

A
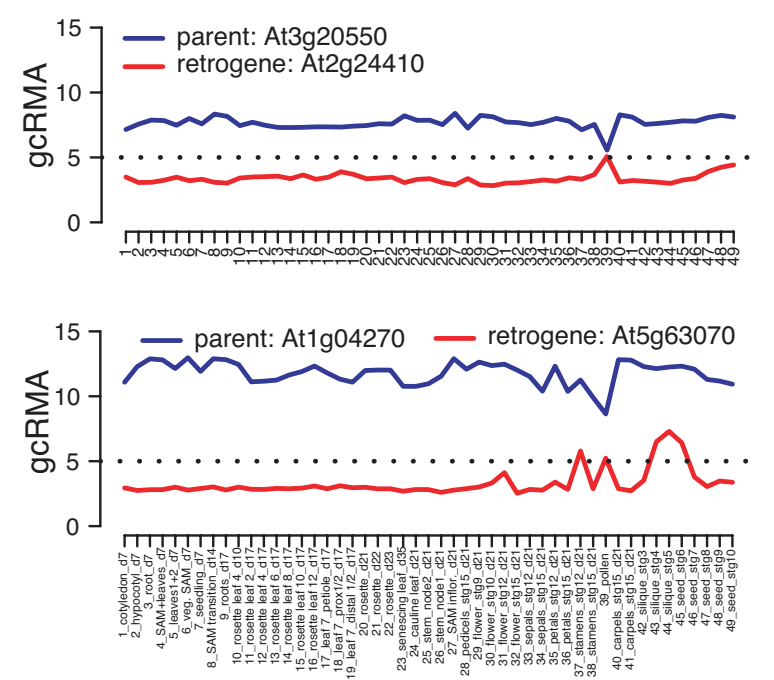

developmental stages

C

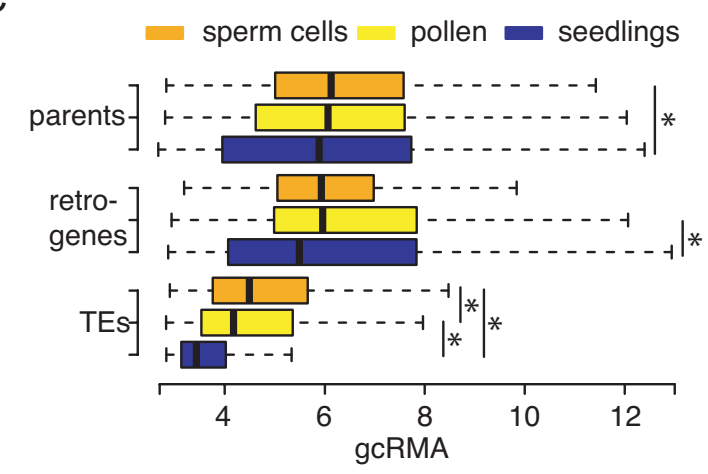

B
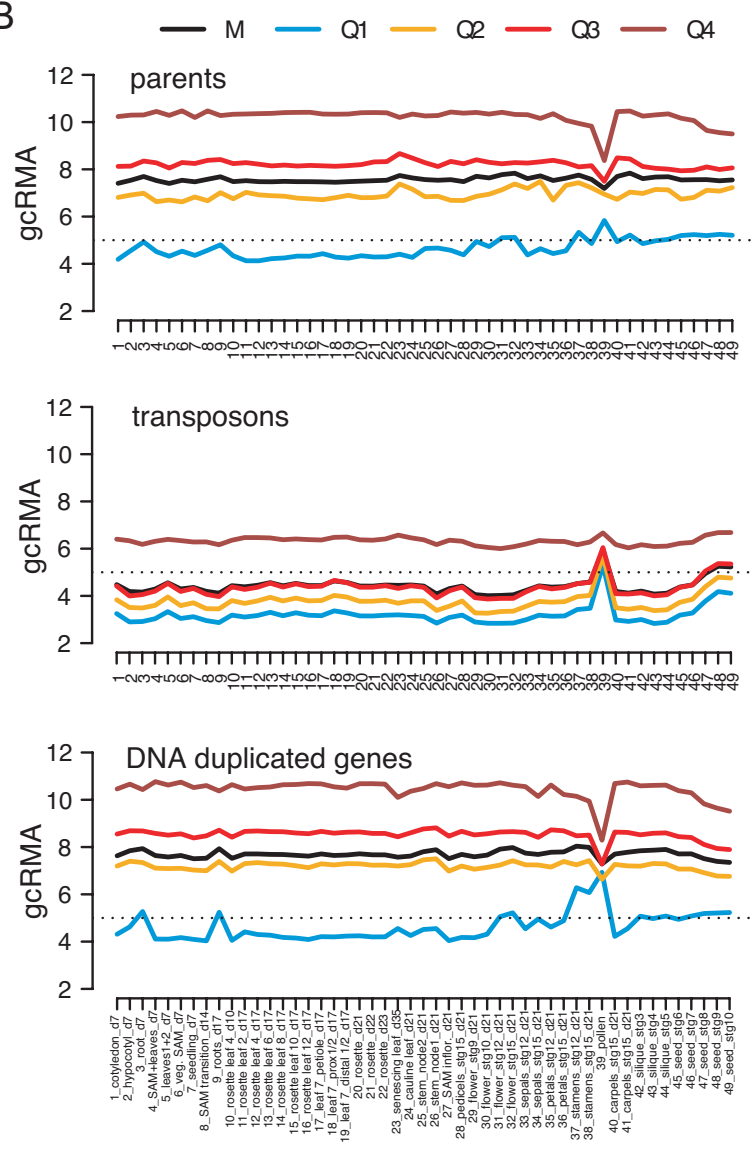

developmental stages

\section{Figure 5. Arabidopsis retrogenes are expressed in pollen}

$(A, B) \log _{2}$ robust microarray averaging (gcRMA) values ( $y$-axis) of specific groups of genes in $49 \mathrm{~A}$. thaliana developmental stages and tissues (x-axis). The horizontal dashed line (gcRMA $=5$ ) indicates the threshold of high expression. (A) Representative examples of retrogene-parent pairs with ubiquitously expressed parents and tissue-specifically expressed retrogenes. (B). gcRMA values for parents (top), transposons (middle) and DNA duplicated genes (bottom) shown as the mean (M) and expression quantiles from low-expressed (Q1) to 
high expressed (Q4). (C) gcRMA expression values for parents, retrogenes and transposons (TEs) in pollen sperm, entire pollen (sperm cells and vegetative cells) and seedlings. Asterisks show significant differences $(P<0.05)$ in Mann-Whitney-Wilcoxon test.

\subsection{Retrogenes are deficient for transcription-permissive chromatin marks in leaf tissues}

Analysis of transcription quantiles suggests that global transcriptional changes in pollen have a major effect on retrogene transcription. This may be achieved by a global chromatin reprogramming (Kaessmann et al., 2009). Therefore, we calculated $\log _{2}$ fold transcription changes between pollen and 21 day-old rosettes (ATGE_73/ATGE_22; Schmid et al., 2005) and correlated those with transcriptional changes induced by chromatin mutants (mutant rosettes/wild type rosettes). Five groups were compared: all genes $(n=22,746)$, pollen up-regulated genes $(n=$ $5,171)$, leaf up-regulated genes $(n=6,057)$, pollen up-regulated retrogenes $(n=51)$ and leaf up-regulated retrogenes $(n=53)$. Tissue up-regulated genes were defined as having $\log _{2}$-fold change $\geq 1$ in one versus the other tissue. First we estimated the effects of the transposon silencing machinery by testing mutants for DECREASED DNA METHYLATION 1 (DDM1), KRYPTONITE (KYP) and HISTONE DEACETYLASE 6 (HDA6) (Baubec et al., 2010; Inagaki et al., 2010; Popova et al., 2013), which lead to loss of repressive DNA methylation and H3K9me2; and gain of permissive histone-acetylation at heterochromatic loci, respectively. There was no clear correlation (maximum $r=0.040$ ) between transcription in pollen relative to leaves and transcriptional changes induced by $d d m 1, k y p$ and hda 6 for all tested groups (Figures $6 \mathrm{~A}-\mathrm{C}$ ). This demonstrates that TE silencing components do not determine the global gene transcription pattern in pollen nor affect retrogenes.

Recently, a connection between pollen-specific genes and H3K27 methylation has been reported in Arabidopsis (Hoffmann and Palmgren, 2013). Therefore, we tested the effects of the histone H3K27me3 mark by analyzing mutants of the polycomb group repressive complex factors CURLY LEAF (CLF) and SWINGER $(S W N)$ that have been shown to control transcription during development (Farrona et al., 2011; Lafos et al., 2011). The correlation between clf and swn single mutants, with pollen-specific transcriptional changes was low $(r<0.20$; Figures 6D,E). Because CLF and SWN are partially functionally redundant (Lafos et al., 2011), we 
tested for effects in the clf/swn double mutant. The correlation between pollen and clf/swn transcription profiles for the set of all genes was higher $(r=0.277)$ than for clf and swn single mutants (Figure 7A; Figure 6D,E). Surprisingly, the high correlation was mainly due to leaf up-regulated genes and retrogenes $(r=0.469$ and 0.364 , respectively) that were coordinately down-regulated in both pollen and clf/swn double mutant (Figure 7A). In contrast, pollen up-regulated genes showed generally uncorrelated transcription with clf/swn $(r=-0.047)$.

A

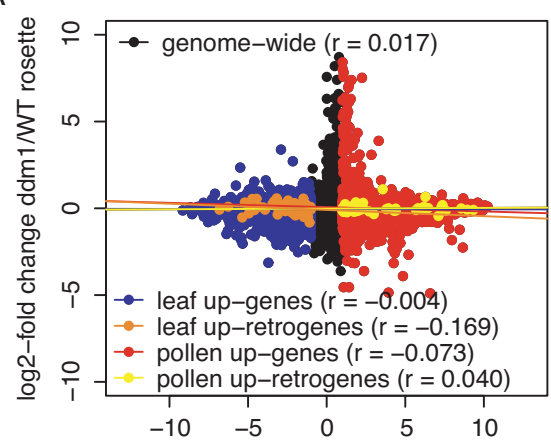

log2-fold change WT pollen/WT rosette

C

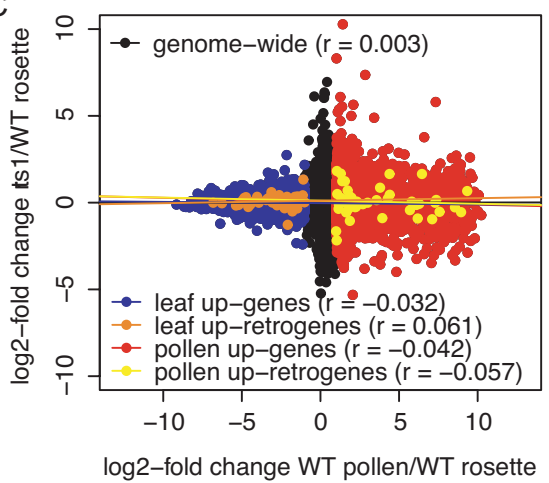

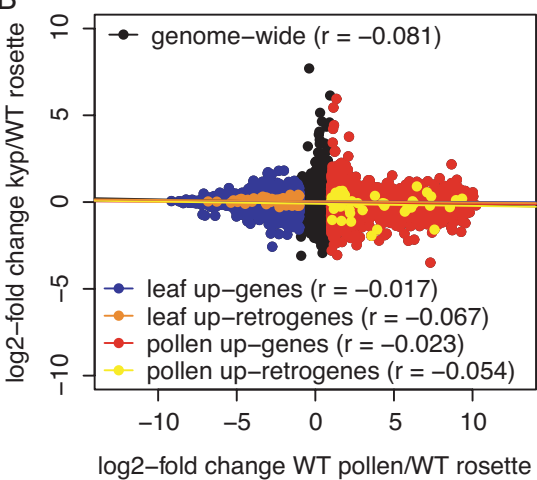

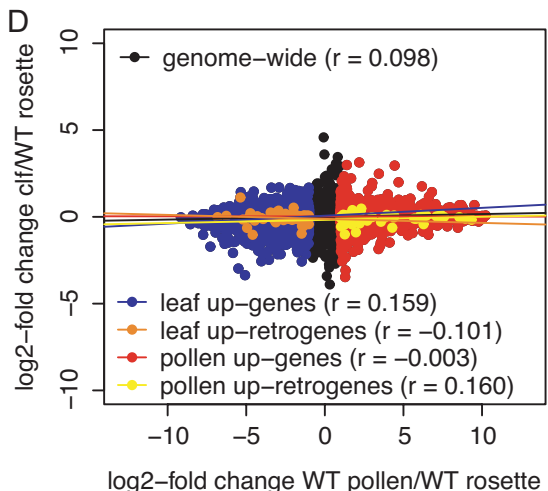

$\mathrm{E}$

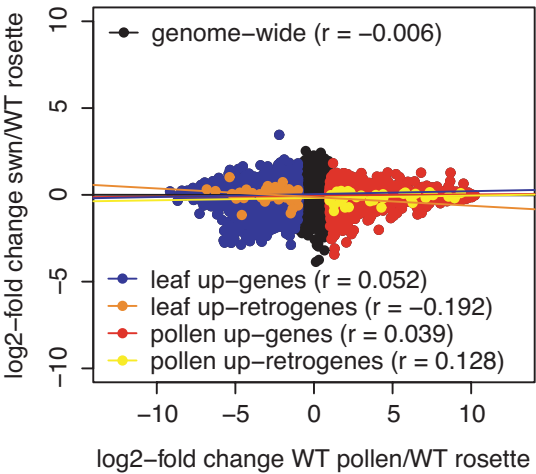

Figure 6. Expression correlations between pollen and chromatin mutants

(A-E) Dot plots of microarray based $\log _{2}$-fold-changes in wild type pollen (ATGE_73)/rosettes (ATGE_22) ( $x$-axis) versus mutant rosettes/wild type rosettes ( $y$-axis). Specific gene sets were superimposed on the genome-wide gene set. The lines indicate expression correlation $(r)$ between the $x$-and the $y$-axis gene sets. (A) shows comparison of pollen with $d d m 1$, (B) with kyp, (C) with HDA6 mutant allele rts1-1, (D) with clf and (E) with swn. 
To further test the connection between pollen-specific transcription and H3K27me3 changes, we analyzed transcription in a mutant for FERTILIZATION INDEPENDENT ENDOSPERM (FIE), another key gene of the Polycomb repressive complex (Bouyer et al., 2011). Although the correlations between fie and pollen transcription profiles were weaker $(r=0.186,0.366$ and 0.268 for all genes, leaf upregulated genes and retrogenes, respectively; Figure 7B), they perfectly recapitulated trends observed in the comparison between clf/swn and pollen. Hence, loss of key components of the Polycomb repressive complex correlates with pollen-specific gene down-regulation of leaf-transcribed genes but does not explain pollen-specific gene up-regulation.

To identify chromatin modification(s) associated with retrogenes and pollenup-regulated genes in somatic tissues, we used publicly available chromatin data from young $A$. thaliana leaves (Roudier et al., 2011). We extracted information on chromatin marks for every gene and compared the full sets of retrogenes, parents and all genes (Figure 7C). In accordance with high and ubiquitous transcription, the parents were enriched for permissive chromatin marks histone $\mathrm{H} 3$ lysine 4 di- and trimethylation ( $\mathrm{H} 3 \mathrm{~K} 4 \mathrm{me} 2$ and me3), histone $\mathrm{H} 3$ lysine K36 tri-methylation ( $\mathrm{H} 3 \mathrm{~K} 36 \mathrm{me} 3)$ and histone $\mathrm{H} 2 \mathrm{~B}$ ubiquitination (H2Bub), followed by retrogenes and the genomewide set. None of these groups was enriched for the repressive H3K27 modifications. The enrichment for gene body DNA methylation in highly expressing genes is consistent with the currently proposed function of this modification (Coleman-Derr and Zilberman, 2012).

The next step was to compare the pattern of chromatin marks distribution for pollen up-regulated and leaf up-regulated genes (Appendix B). The distribution pattern of chromatin marks for each individual group (retrogenes, parents and all genes) was relatively similar (Figure 7D, Figures 8A,B). There were no changes in gene body DNA methylation. While the H3K27 modifications were enriched in pollen up-regulated genes of the genome-wide set, this mark does not seem to play a major role in somatic silencing of pollen up-regulated retrogenes (Figure 8A). In contrast, all analyzed transcription-permissive marks (H3K4me2 and me3, H2Bub and H3K36me3) were underrepresented in pollen up-regulated genes in leaf tissues (Figure 7D, Figures 8A,B). The presence or the absence of these marks was strongly correlated in pair-wise comparisons of individual modifications (Figure 7E, Figure $8 C)$. 
This suggests that in leaf tissues, retrogenes and other pollen up-regulated genes are depleted of permissive chromatin marks without enrichment for repressive marks. In contrast, leaf up-regulated genes are down regulated in pollen by a mechanism involving the Polycomb repressive complex components CLF, SWN and FIE.

A

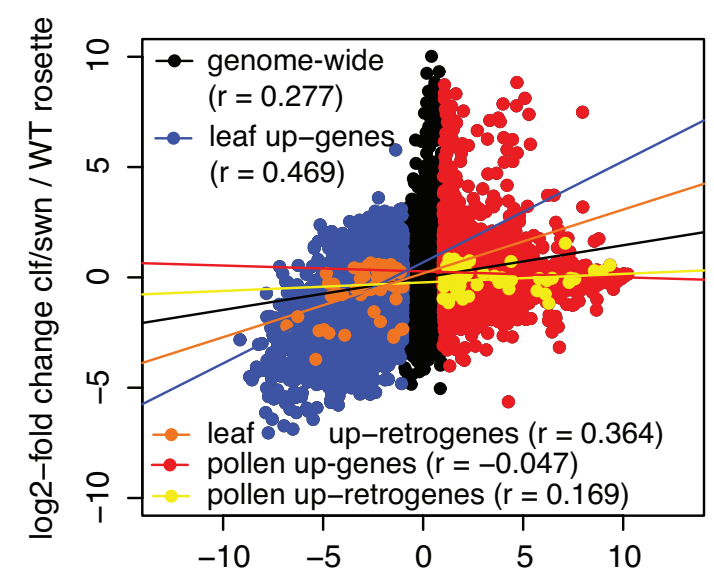

log2-fold change WT pollen/WT rosette

B

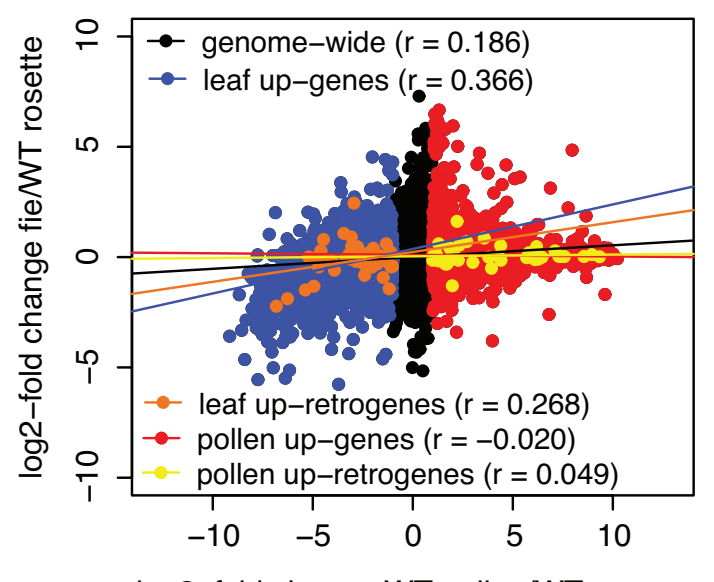

log2-fold change WT pollen/WT rosette

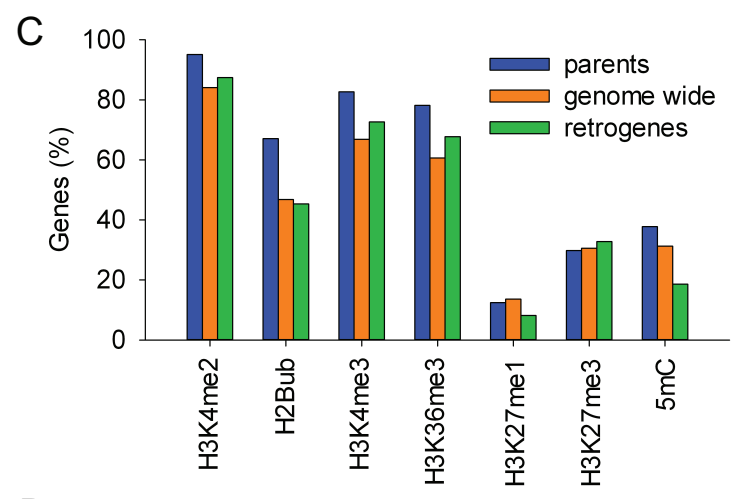

D 100$]$

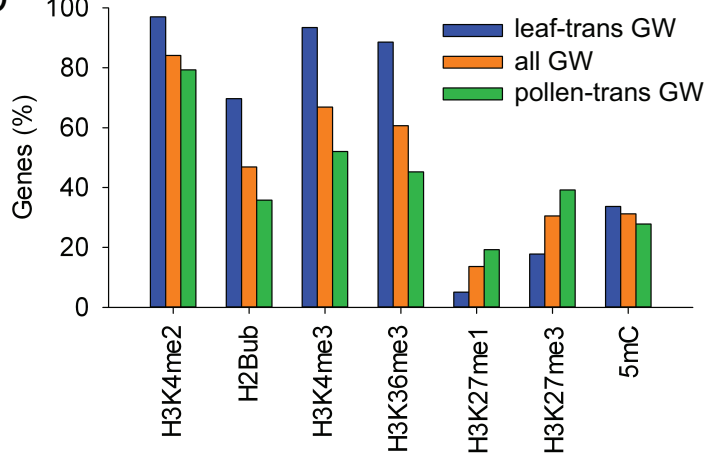

E

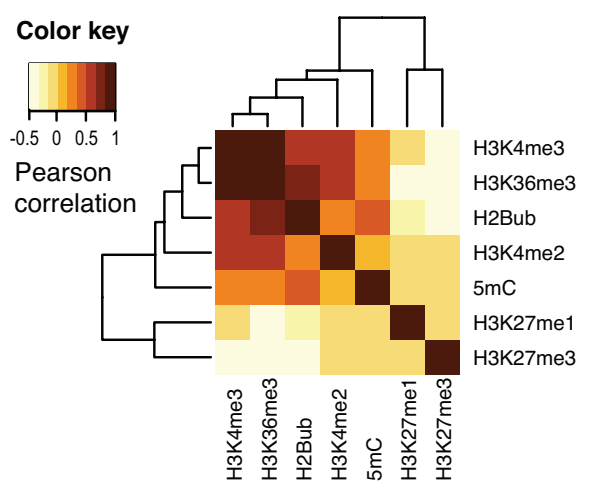

Figure 7. Chromatin control of pollen-specific gene transcription

(A-B) Dot plots of $\log _{2}$-fold changes in wild type pollen/rosettes ( $x$-axis) and (A) clf/swn double mutants or (B) fie/wild type rosettes ( $y$-axis). Specific gene sets were superimposed on the genome-wide set in different colors. Lines indicate transcription correlation ( $r$ ) between the $x$ - and the $y$-axis for specific gene sets. The $r$ values are given in parentheses. (C) The frequency of seven chromatin modifications at gene coding sequences for GW, $P$ and $R$ in young leaf tissues. (D) The same as (C) for all genes (all GW), leaf-transcribed genes (leaf-trans GW) and pollen-transcribed genes (pollen-trans GW). (E) Hierarchical clustering and heat map of Pearson correlation values of co-localization between 7 chromatin modifications for all $A$. thaliana genes. 

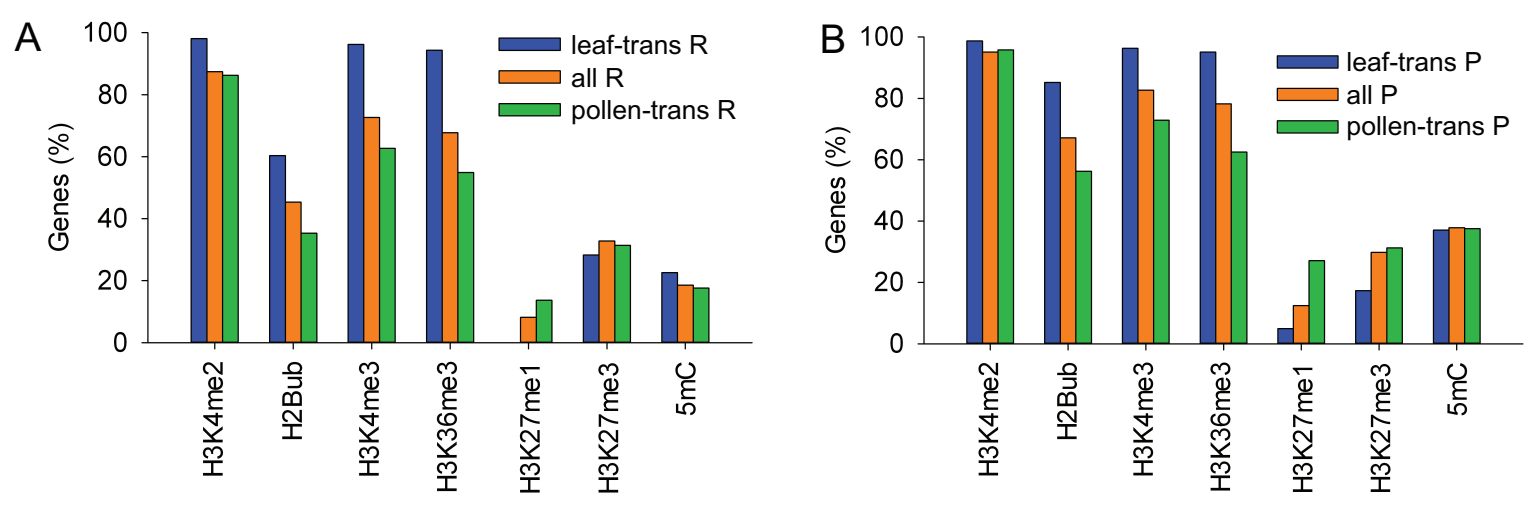

C
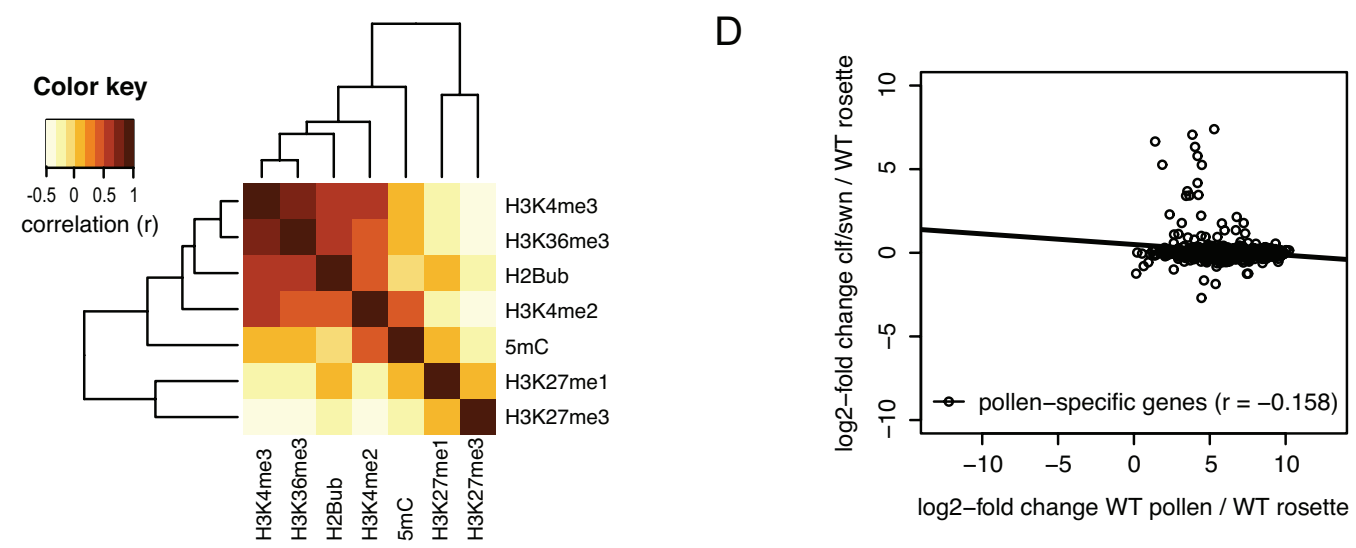

Figure 8. Chromatin control of pollen-specific gene expression

(A) The frequency of seven chromatin modifications at protein coding regions for all retrogenes (all $R$ ), leaf-expressed retrogenes (leaf-expr $R$ ) and pollen-expressed retroges (pollen-expr R) in young leaf tissues. (B) Shows the same as (A) but for parents. (C) Hierarchical clustering and heat map of Pearson correlations between seven analyzed chromatin modifications for all retrogenes. (D) Dot plot of $\log _{2}$-fold-changes in wild type pollen (ATGE73)/rosettes (ATGE_22) ( $x$-axis) versus clf/swn doublemutant/wild type rosettes ( $y$-axis) for the set of 584 pollen-specific genes defined by Hoffmann and Palmgren, 2013. The black line indicates expression correlation $(r)$ between the $x$-and the $y$-axis datasets.

\subsection{Gain of transcription factor binding sites facilitates PCR11 retrogene sperm-specific transcription}

Retrogenes showed gamete-specific transcriptional activation. Therefore, we tested whether gamete-specific transcription of retrogenes has evolved into gametespecific developmental functions. Five retrogenes found in our screen MULTICOPY SUPRESSOR OF IRA1 (MSI1), PLANT CADMIUM RESISTANCE 11 (PCR11), BETA GLUCOSIDASE 14 (BGLU14), MATERNAL EFFECT EMBRYO ARREST 25 (MEE25) and PEROXIDASE are associated with pollen development, sperm cell 
differentiation, pollen tube growth and development (TAIR10). To test whether these retrogenes have evolved parent-independent gamete-specific expression and function, we investigated the relationship between transcription of these retrogenes and their parents. We plotted their mean developmental gcRMA values and calculated transcription Pearson correlations (Figs 9A-D). The parental gene of the PEROXIDASE was not included on the ATH1 array and therefore we did not continue its analysis. The transcription of MS/1 was strongly correlated $(r=0.905)$ with its parent MSI4 and both were ubiquitously transcribed throughout development (Figure 9A). BGLU14 and its parent BGLU15 were both up-regulated in pollen (Figure 9B). The MEE25 retrogene was lowly transcribed during the entire development and higher transcription was found only in embryonic tissues (Figure 9C). However, its parent, At4g10960, was transcribed mainly in floral tissues, seeds and pollen where it greatly surpassed MEE25 transcription. Hence, these three retrogenes did not provide evidence for development of parent-independent pollen-specific transcription. In contrast, PCR11 was lowly transcribed almost throughout entire development, but activated in floral tissues, stamen and pollen. This pattern was opposite to that of its parent, $P C R 2$, which was active mainly in the photosynthetically active tissues and down regulated in stamen and pollen (Figure 9D). The PCR11 gene is transcribed specifically in pollen sperm cells by the MYB-transcription factor DUO1 (Borg et al., 2011). Therefore, we compared promoter regions of PCR11 and PCR2 and looked for previously described DUO1 binding motifs (Borg et al., 2011). There are three binding regions in the $500 \mathrm{bp}$ region upstream of the PCR11 TSS (TAACCGTC at -47 to -54 bp and AAACCG at -153 to -158 and -452 to $-457 \mathrm{bp}$ ). However, only a single DUO1 binding motif (AAACCGT at -100 to $-106 \mathrm{bp}$ from the TSS) is found in the promoter of $P C R 2$. To test whether this represents gain of function in PCR11 or loss of function in $P C R 2$, we compared promoter regions of several other $P C R$ family members representing both the PCR2 clade (PCR1, PCR3) and the out-groups (PCR4, PCR8, PCR10) (Song et al., 2010). None of these genes contained a single DUO1 binding motif in the 500 bp region upstream of the TSS. Furthermore, comparing their transcript levels revealed that only PCR11 is significantly up regulated in pollen relative to $P C R 2$ (Figure $9 \mathrm{E}$ ).

To test these results in an independent experiment, we analyzed retrogene and parent transcription in $A$. thaliana lines carrying somatically inducible DUO1 (Borg et al., 2011). Upon 6, 12 and 24 h DUO1 induction, we observed 36, 131 and 
125 significantly up regulated and 47, 124 and 121 significantly down-regulated genes, respectively. The number of up- and down-regulated retrogenes (2 and 1, respectively) was small, showing that DUO1 controls transcription of only few specific retrogenes. Importantly, the set of significantly up-regulated retrogenes included $P C R 11$ retrogene (log2-fold changes in 6, 12 and 24 h: 0.26, 2.21 and 4.03; t-test $P$ values: $0.010,3.3 \times 10^{-5}, 5.4 \times 10^{-5}$; respectively). This has been reflected by significant down-regulation of its parent $P C R 2$ in two out of three experimental points (log2-fold changes in 6, 12 and $24 \mathrm{~h}:-1.18,-1.60$ and -0.74; t-test $P$ values: 0.003 , $0.007,0.19$; respectively). Therefore, we conclude that the $P C R 11$ retrogene gained sperm cell-specific DUO1-dependent transcription independent of its parent PCR2.
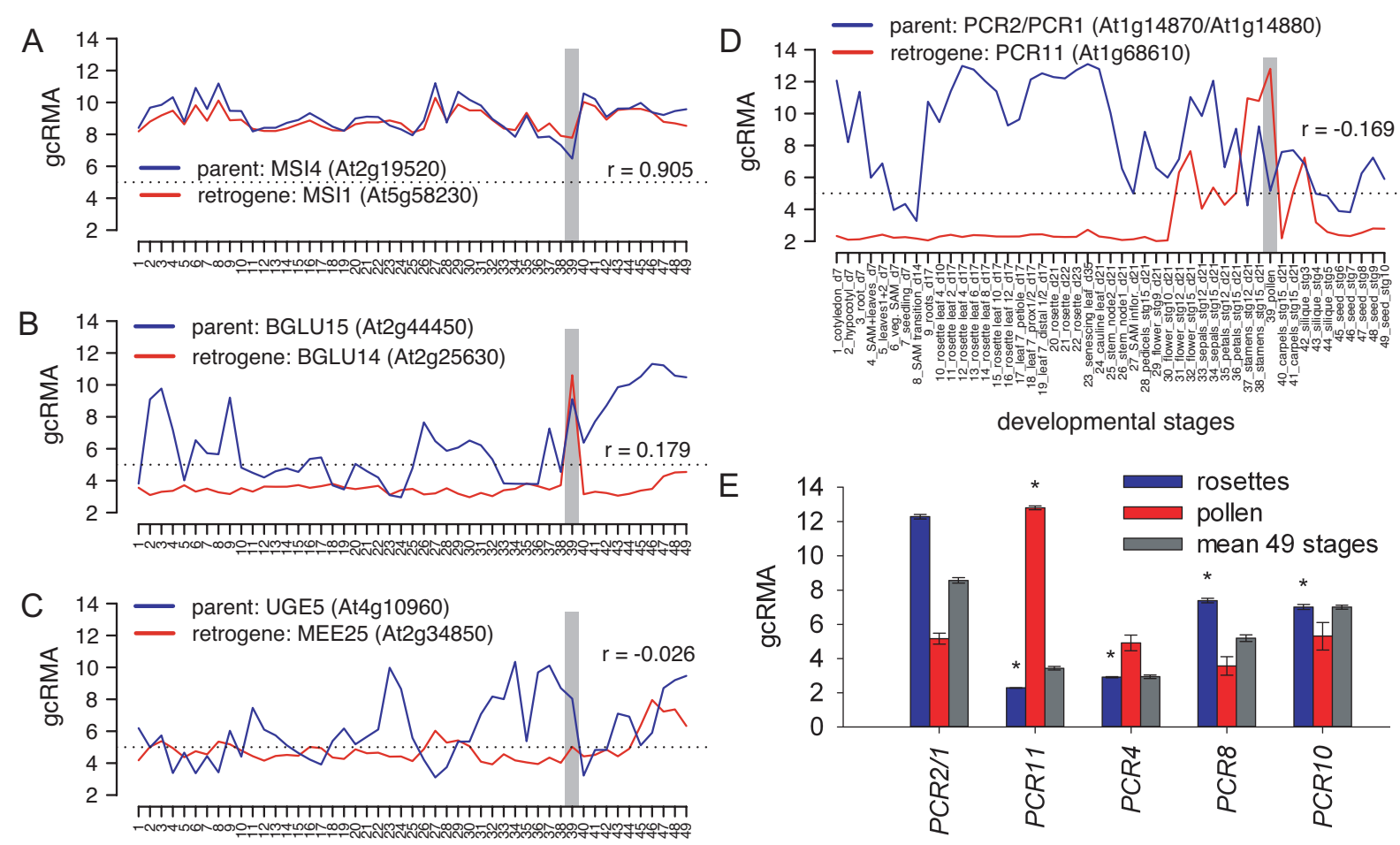

Figure 9. Gain of pollen-specific transcription by PCR11 retrogene

(A-D) Developmental gcRMA transcription profiles of retrogenes associated with pollen growth and development and their parents. Pollen stage is highlighted by vertical gray bar. (E) gcRMA transcription values of $P C R$ family genes in rosettes, pollen and mean of 49 developmental stages and tissues. PCR2 and PCR1 correspond to single microarray element and therefore are shown together. Transcription values were compared to PCR2/PCR1 transcription in the same tissue and statistically analyzed by t-test. Error bars denote standard deviation of three biological replicates. 


\subsection{Improving gene structure annotation of $A$. lyrata genome using RNA- seq data}

We aimed to identify $A$. lyrata retrogenes to study their genomic features, regulation and evolution in comparison to those of $A$. thaliana. Therefore, we used the RAT to screen for retrogenes in the published genome annotation of $A$. lyrata subsp. lyrata accession MN47 (Grigoriev et al., 2012; Hu et al., 2011). However, we noticed major structural differences in many $A$. lyrata genes compared to their orthologs in $A$. thaliana. This was later confirmed to be due to an inaccuracy in $A$. lyrata genome annotation version 1 , which was based almost exclusively on in silico prediction tools (Hu et al., 2011). Two major observed annotation inaccuracies were: 1) merging two neighbor genes into one gene model (Figure 10A), and 2) splitting a single gene into two gene models (Figure 10B). This suggested that A. lyrata genome annotation v1, cannot be used for precise retrogene mapping. To correct the inaccuracies and enhance the overall structural annotation of $A$. lyrata genome, we generated deep transcriptome sequencing (RNA-seq) data to guide our in silico prediction of $A$. lyrata gene models (Figure 10). We used RNA samples from rosettes, inflorescences and shoot apical meristem tissues from plants grown under ambient conditions, for deep sequencing (Illumina technology). Additionally, RNA sequencing reads from tissue samples grown under heat and cold stress conditions were provided by Pecinka lab and Weigel lab at Max Planck Institutes. All grouped RNA-seq reads were mapped against the $A$. lyrata reference genome using Bowtie2/Tophat2 (Kim et al., 2013; Langmead and Salzberg, 2012) and used to direct the in silico prediction of gene models using AUGUSTUS software (Stanke et al., 2008; Stanke et al., 2006) (Figure 10). Reverse transcription PCR data further confirmed gene structure prediction. Our annotation, version 2, showed improved estimation of annotated coding regions as well as gene and exon length (Table 2). Additionally, genes were named following $A$. thaliana unified gene nomenclature. Gene model annotation was done in collaboration with Schneeberger lab at MPIPZ (Rawat, Abdelsamad et al., submitted for publication). This version of annotation (v2) was then used for identification of retrogenes. 
A

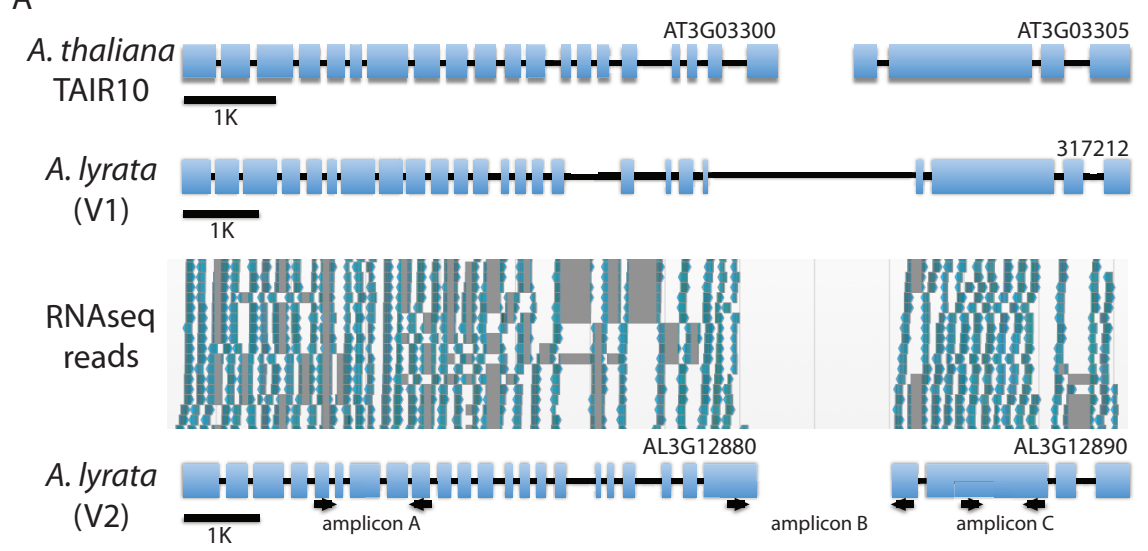

B

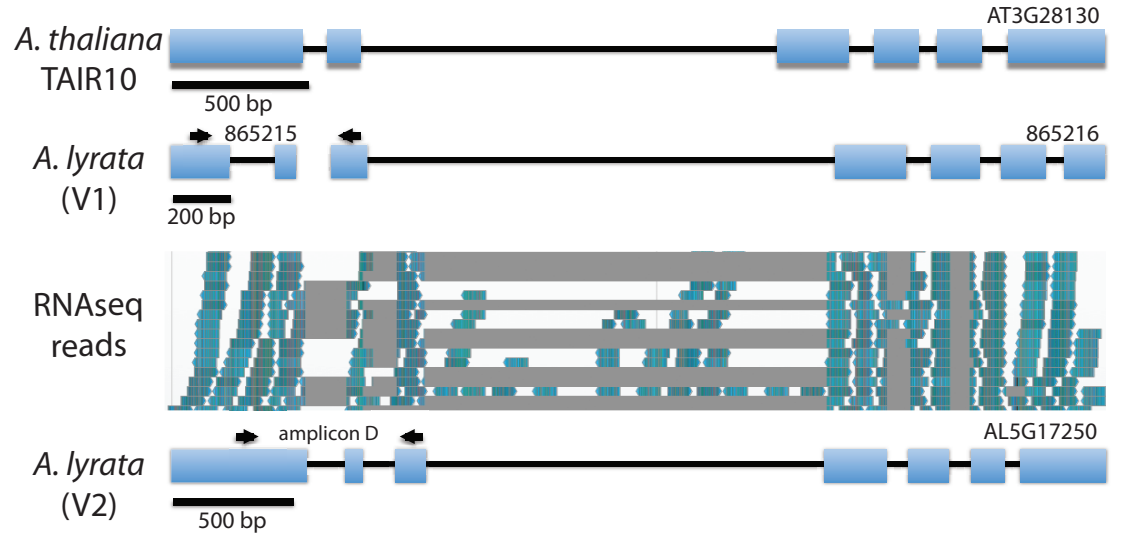

Figure 10. Enhancement of $A$. lyrata gene models using RNA-seq

(A) Example of inaccurate fusion of two neighboring gene models in annotation (V1), and its correction in our improved version (V2) using RNA-seq reads as guide for prediction. (B) Example of inaccurate split of a gene model into two neighboring gene models in annotation (V1), and its correction in our improved version (V2) using RNA-seq reads as guide for prediction. Black arrows indicate primer positions for reverse transcription PCR amplicons that support our predicted gene models.

Table 2. Comparison of $A$. lyrata annotation (version 2) to version 1 and TAIR10

\begin{tabular}{lccc}
\hline & $\begin{array}{c}\text { A. thaliana } \\
\text { TAIR10 }\end{array}$ & $\begin{array}{c}\text { A. lyrata } \\
\text { Version 1 }\end{array}$ & $\begin{array}{c}\text { A. lyrata } \\
\text { Version 2 }\end{array}$ \\
\hline Genome size (Mbp) & 120 & 207 & 207 \\
Coding portion (Mbp) & $65(54 \%)$ & $39(19 \%)$ & $79(38 \%)$ \\
Protein coding genes & 27416 & 32670 & 31606 \\
Average gene length (bp) & 2122 & 1192 & 2496 \\
Average peptide length (bp) & 1855 & 1085 & 1244 \\
Total exon length (Mbp) & 50 & 39 & 51 \\
Average exon no. per gene & 4.7 & 5.3 & 5.5 \\
Average exon size (bp) & 328 & 223 & 291 \\
\hline
\end{tabular}




\subsection{Fast emergence of Arabidopsis retrogenes revealed by interspecies comparison}

We used RAT to conduct a genome-wide screen for retrogenes in our improved version of $A$. lyrata genome annotation. The screen was based on global gene paralogy search among non-transposable element protein coding genes, followed by search for retrogene-specific features among primary retrogene candidates (Figure 11B). Applying stringent quality criteria, our tool identified 168 putatively functional retrogenes (Appendix D), which represent $0.53 \%$ of $A$. lyrata protein coding genes $(n=31,606)$.

The close taxonomic relationship between $A$. thaliana and $A$. lyrata opened the opportunity to investigate evolution of retrogenes identified by RAT tool in both species (Table 3 ). Therefore, we established genome-wide gene orthology relationships among $A$. thaliana, $A$. lyrata and outgroup species Capsella rubella (Figure 11A). We used Inparanoid v4.01 (Remm et al., 2001) to establish gene orthology, based on protein sequence similarity. Among the three used genome annotations, pseudogenes are only annotated in $A$. thaliana genome hindering the efforts to identify the orthologs of this category in the other genomes. We specifically investigated retrogenes, of which $157(62 \%)$ and $147(86 \%)$ in $A$. thaliana and $A$. lyrata, respectively, had orthologs in the second species and/or in the out-group. This supports their conservation and origin before split from the last common ancestor (Figure 11C). For $51(20 \%) A$. thaliana and 23 ( 14\%) A. lyrata retrogenes, we couldn't find orthologs in the second species or C. rubella; i.e. these retrogenes were species-specific. The nucleotide similarity between these species-specific retrogenes and their parental genes was significantly higher than the nucleotide similarity between pre-split retrogenes and their parental genes, as shown by MWW test, $\mathrm{P}=$ $1.479 \mathrm{e}-07$ and 0.1053 in A. lyrata and A. thaliana, respectively (Figure 11C). This supported their retroposition post the split of both species from the last common ancestor. The observed post-split retroposition events estimated an evolutionary rate of retrogene emergence of 5-10 retrogenes per million years.

Interestingly, some of pre-split and post-split retrogenes originate from the same parental gene. Like for $A$. thaliana we observed repeated secondary retroposition events; i.e. when a primary parent gives rise to a primary retrogene whose mRNA serves as the precursor for a secondary retrogene. Table 4 lists six 
retrogenes whose mRNAs were precursors for seven secondary retrogenes, one of which happened after the split. We also identified parental genes that gave rise to multiple retrogene copies. In total six parents gave rise to fifteen retrocopies $(4 \times 2,1$ $\times 3,1 \times 4$ ). While most of them retroposed before the split (pre-split), a single gene (AL7G18010) gave rise to three retrocopies post split (Appendix D). The observed frequency of multiple retroposition events from the same parental gene is significantly higher than expected at random (MWW test, $\mathrm{P}<0.05)$.

A

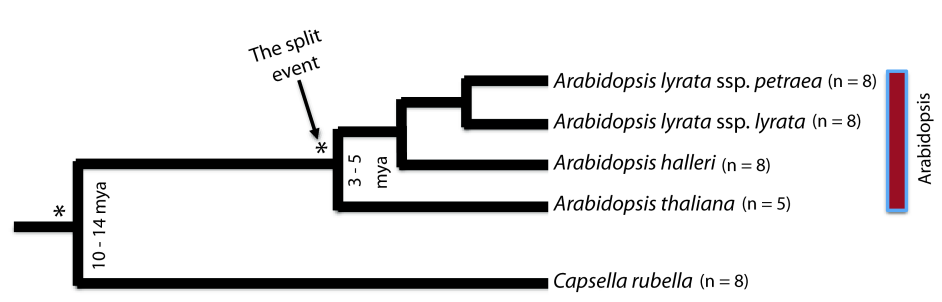

C
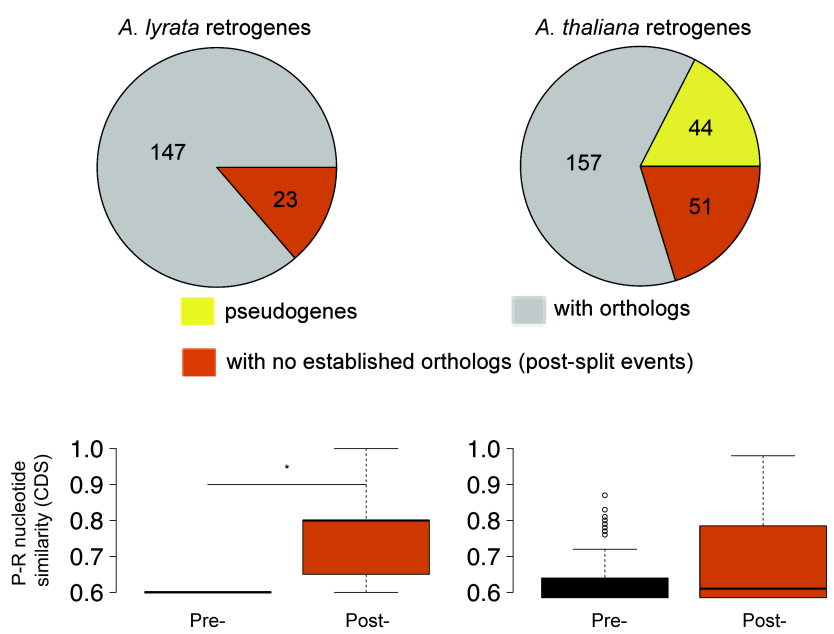

B

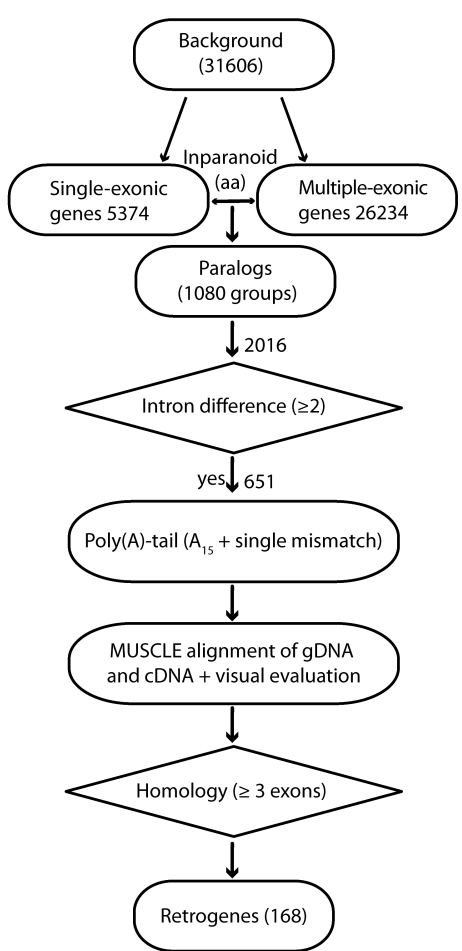

Figure 11. Novel identification of $A$. lyrata retrogenes and the relatively recent emergence

(A) A schematic strict consensus tree of some Arabidopsis species. C. rubella serves as an outgroup. The tree illustrates the relatively early divergence of $A$. thaliana from other Arabidopsis species. Abbreviation: mya, million years ago. Figure modified from (Clauss and Koch, 2006; Yogeeswaran et al., 2005) (B) Schematic representation of retrogene identification in A. lyrata genome (Abdelsamad and Pecinka, 2014). (C) Pie charts present orthology-based classification of $A$. lyrata and $A$. thaliana retrogenes into: 1. Pre-split retrogenes with an ortholog in the other species and/or the outgroup (grey), 2. Post-split retrogenes with no orthologs in the other species or the outgroup (orange), and 3 . Pseudogenes (yellow). Graphs show parent-retrogene (P-R) coding region (CDS) nucleotide similarity for pre- and post-split retrogenes. 
Table 3. Total (conserved) retrogenes and parental genes identified by RAT tool

\begin{tabular}{lcccc}
\hline & \multicolumn{2}{c}{ A. lyrata } & \multicolumn{2}{c}{ A. thaliana } \\
& Parents & Retrogenes & Parents & Retrogenes \\
\hline $\begin{array}{l}\text { Has ortholog in the other species } \\
\text { and/or C. rubella }\end{array}$ & 147 & 145 & 195 & 157 \\
$\begin{array}{l}\text { No established orthologs in the } \\
\text { other species or in C. rubella }\end{array}$ & 12 & 23 & 33 & 51 \\
$\begin{array}{l}\text { Pseudogenes } \\
\text { Total }\end{array}$ & $\mathbf{1 5 9}$ & $\mathbf{1 6 8}$ & $\mathbf{2 2 8}$ & $\mathbf{2 5 2}$ \\
\hline
\end{tabular}

Table 4. Repeated retroposition events in A. lyrata

\begin{tabular}{cccccc}
\hline \multicolumn{2}{c}{ Parents } & \multicolumn{2}{c}{$1^{\text {st }}$ retroposition } & \multicolumn{2}{c}{$2^{\text {nd }}$ retroposition } \\
Gene ID & Introns & Gene ID & Introns & Gene ID & Introns \\
\hline$A L 6 G 33170$ & 27 & $A L 1 G 36880$ & 17 & $A L 1 G 44670$ & 9 \\
& & & & $A L 3 G 30910$ & 0 \\
$A L 3 G 33920$ & 9 & $A L 1 G 40380$ & 3 & $A L 3 G 35610$ & 0 \\
$A L 3613250$ & 4 & $A L 4 G 19410$ & 2 & $A L 1 G 17240^{*}$ & 0 \\
$A L 2 G 21490$ & 9 & $A L 7 G 13720$ & 6 & $A L 5 G 23850$ & 0 \\
$A L 7 G 22160$ & 14 & $A L 7 G 15950$ & 5 & $A L 5 G 15220$ & 0 \\
\hline
\end{tabular}

* Post-split event

Hence, we report the first of $A$. lyrata retrogenes (168), $86 \%$ of which had orthologs in $A$. thaliana and/or C. rubella. About 3.8\% of parents gave rise to multiple retrogenes, some of which occurred after the split from common ancestor of both species; and $\sim 3.6 \%$ of the retrogenes underwent a second retroposition without loss of functionality. About $14 \%$ and $20 \%$ of $A$. lyrata and $A$. thaliana retroposition events, respectively, occurred after the split between the two species corresponding to 5-10 successful retroposition events per million year.

\subsection{Arabidopsis retrogenes and transposable elements share amplification mechanism but not chromosomal location and transcriptional regulation}


Retrogene precursor mRNA molecules are reverse transcribed and integrated in the genome by the same enzymatic machinery that duplicates retrotransposons and retroviruses (Kaessmann et al., 2009). Many retrotransposons are integrated in repeat-rich regions, which affect their transcription and silencing (Tsukahara et al., 2012). To investigate the pattern of retrogene integration, we plotted the densities of retrogenes, parents, TEs and non-TE protein-coding genes over the eight largest scaffolds of $A$. lyrata genome assembly $\mathrm{v} 1$ representing the eight pseudochromosomal molecules of $A$. lyrata genome (Figure 12). In agreement with their distribution over $A$. thaliana chromosomes (Figure 2A), retrogenes and their parents show overall distribution profiles similar to that of protein-coding genes (GW) and different from that of TEs. Unlike for $A$. thaliana chromosomes (Figure 2A), the current assembly of $A$. lyrata scaffolds (Grigoriev et al., 2012; Hu et al., 2011) does not include centromeric regions; and thus, the distinguished enrichment of TEs at the centromere cannot be clearly seen in Figure 12. Hence the overall distribution of parents and retrogenes in both genomes follow that of other non-TE protein coding genes.
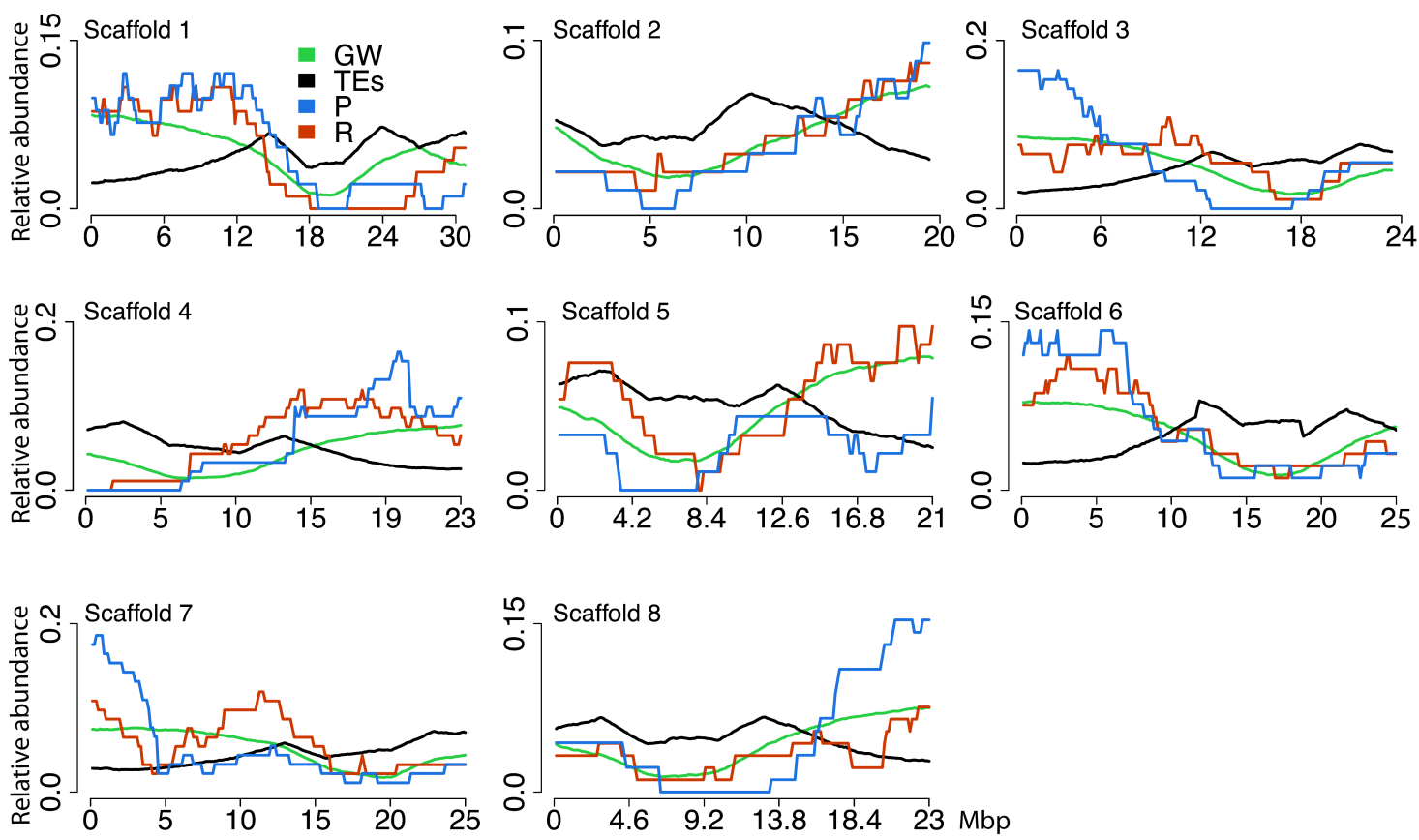

Figure 12. Distinctive chromosomal location of retrogenes and TEs

Retrogenes (R; orange) and parents $(P$; blue) show relative abundance ( $y$-axis) over the eight main scaffolds ( $x$-axis) of $A$. lyrata similar to non-TE protein coding genes (GW; green) and different from TEs (black). 
To further investigate the local association of parents and retrogenes with TEs at single gene resolution, we calculated the frequency of genes overlapping with TEs, or flanked by TEs in one kilobase (1-kb) intervals upstream and downstream of gene transcription start sites (TSSs) and gene transcription termination sites (TTSs) respectively (Figure 13A). Generally, there were slightly fewer genes with TEs in their downstream regions than genes with TEs in their upstream regions. On average, there is a non-significantly lower frequency of retrogenes with flanking TEs compared to parents and genome wide genes. However, retrogenes that overlap with TEs are significantly scarce compared to genome wide protein coding genes, $4 \%$ and $11 \%$, respectively (chi-square test, $\mathrm{P}<0.05$ ); and that might be explained by the significantly shorter average retrogene length compared to GW genes (Figure 13D). We further investigated the local surroundings of retrogenes by plotting their intergenic distances (Figure 13B). Although A. lyrata has longer intergenic distances and lower gene density than $A$. thaliana, retrogenes retain their preference for occurrence in gene-rich genomic regions with relatively short intergenic regions. This is similar to parents and other protein-coding genes. Hence, the local distribution of retrogenes indicates their preferential integration in gene-rich regions that are not enriched for TEs.

Retrotransposable elements transpose together with their regulatory sequences that drive their expression post integration. On the contrary, retrogenes are supposed to transpose, through a mature mRNA intermediate, without upstream regulatory regions. We wanted to explore the expression behavior of retrogenes in comparison to TEs and non-TE protein coding genes. Using our data of deep transcriptome sequencing, we calculated transcription values as a sequencing read per kilobase per million reads (RPKM). The genetic element was considered expressed if RPKM $\geq 1$ in at least one tissue type, developmental stage or stress condition under investigation. We plotted transcription values (RPKM) for transcribed genes; i.e. RPKM $\geq 1$ (Figure $13 \mathrm{C}$ ), $84 \%$ and $90 \%$ of retrogenes and other non-TE protein coding genes (GW) were expressed at non-significantly different levels (MWW test, $P=0.702$ ). In contrast, only $28 \%$ of TEs were expressed; and their expression was at levels significantly lower than for protein coding genes (MWW test, $P=0.0017$ ). On the contrary, $96 \%$ of parental genes are expressed at significantly higher levels than genome wide genes, retrogenes and TEs (MWW test, $P=3.573 \mathrm{e}-$ $06,0.0067$ and $8.149 \mathrm{e}-07$ respectively). Hence, the frequency of expressed 
retrogenes and their expression levels are higher than for TEs and mirror genome wide genes.

The cell could consider retrogene copies as dispersed repeats, based on their repeated nature and TE-like duplication mechanism. Therefore, silencing small RNA molecules (sRNAs) might regulate retrogenes transcription in a pattern similar to TE regulation. We calculated the number of gene-specific and TE-specific $21 \mathrm{nt}$ and $24 \mathrm{nt}$ sRNA molecules, respectively, per kilobase of each retrogene, parent, genome-wide genes, retrotransposons and DNA transposons. Retrogenes and genome-wide genes are targeted by $21 \mathrm{nt}$ sRNAs at non-significantly different ratios (MWW test, $\mathrm{P}$ $=0.489$ ); however, significantly higher than retrotransposons and DNA transposons (MWW test, $\mathrm{P}<2.2 \mathrm{e}-16$ and $\mathrm{P}=3.107 \mathrm{e}-16$, respectively) (Figure 13E). On the other hand, ratios of $24 \mathrm{nt}$ sRNA targeting retrogenes are significantly lower than ratios for retrotransposons and DNA transposons (MWW test, $\mathrm{P}=9.914 \mathrm{e}-14$ and $\mathrm{P}=1.439 \mathrm{e}-$ 12 , respectively), but non-significantly different from genome-wide genes (MWW test, $P=0.125)$ (Figure 13F). Interestingly, the category of parental genes is targeted by significantly more $21 \mathrm{nt}$ sRNA than retrogenes and genome-wide genes (MWW test, $\mathrm{P}$ $=0.00616$ and $\mathrm{P}=0.00069$, respectively); i.e. $21 \mathrm{nt}$ sRA are targeting parent genes at the highest density among all categories of genetic elements (Figure 13E).

A

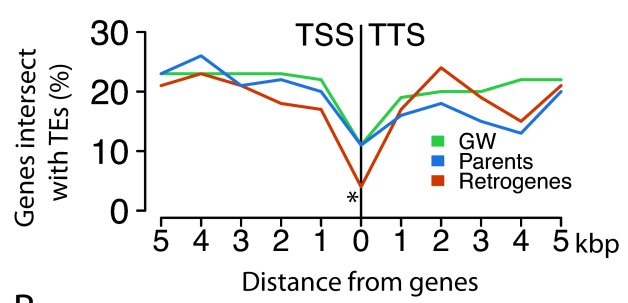

B

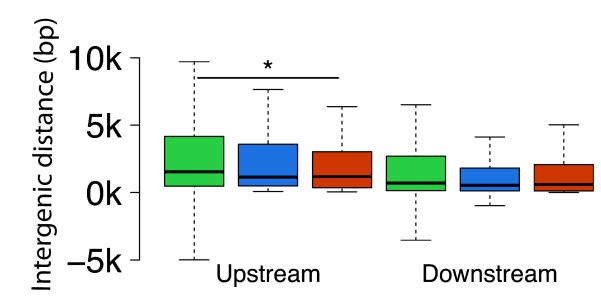

C

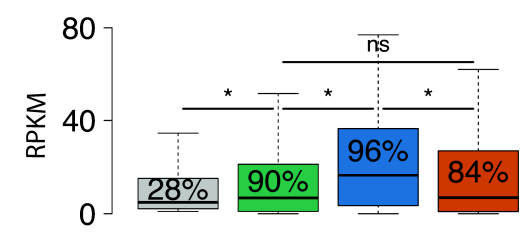

D

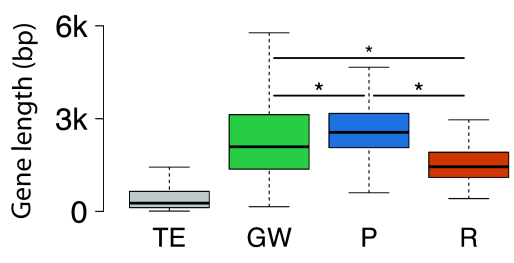

$E$

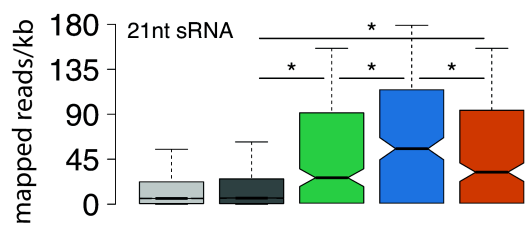

$\mathrm{F}$

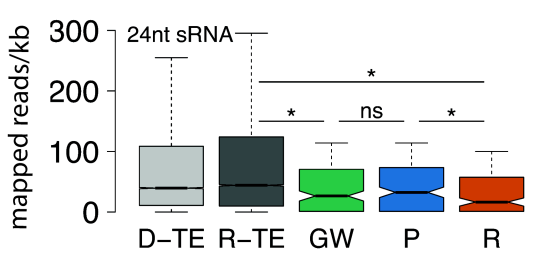

Figure 13. Retrogenes are not integrated, expressed or regulated like TEs 
(A) Percentage of retrogenes overlapping with TEs ( $y$-axis) in $1 \mathrm{kbp}$ intervals from the gene transcription start and termination sites (TSS and TTS, respectively) is not significantly different from all non-TE protein coding genes (GW) and parents. Significant differences $(P<$ 0.05 ) in $X^{2}$-test relative to $\mathrm{GW}$ are indicated by asterisk. (B) Retrogenes are preferentially inserted in gene-rich genomic regions flanked by similar or less intergenic distance in bp $(y-$ axis) to/than GW and parents. Significant $(P<0.05)$ difference in MWW test is indicated by asterisk. (C) Boxplots of mRNA sequencing reads per kilobase per million reads (RPKM) show non-significantly different expression of retrogenes and genome-wide protein coding genes, while parents are expressed at significantly higher levels. Meaningful significant $(P<$ $0.05)$ and non-significant $(P \geq 0.05)$ comparisons in MWW test are indicated. (D) Boxplots of gene length indicate that retrogenes are significantly shorter than GW and parents. Meaningful significant $(P<0.05)$ comparisons in MWW test are indicated. (E, F) Absolute numbers of mapped regulatory $21 \mathrm{nt}$ sRNA reads $(\mathrm{E})$ and $24 \mathrm{nt}$ sRNA $(\mathbf{F})$ per kilobase $(\mathrm{kb})(y$ axis) indicate that retrognes ( $R$, orange), parents $(P$, blue) and all protein coding genes ( $G W$, green) are regulated by significantly more and significantly less gene-specific and TE-specific regulatory $21 \mathrm{nt}$ sRNA and 24nt sRNA, respectively, than retrotransposons (R-TE, dark grey) and DNA transposons (D-TE, light-grey). The most meaningful significant $(P<0.05)$ and non-significant $(P \geq 0.05)$ comparisons in MWW test are indicated.

\subsection{NRPD2E2 ${ }^{\mathrm{Aly} \_\mathrm{MN} 47}$ : an unusual retrogene in A. lyrata genome}

We identified the $A$. lyrata ortholog of $A$. thaliana NRPD2E2 gene. The gene encodes for the second largest subunit of NUCLEAR RNA POLYMERASE IV 2N 2 (NRPD2E2); and is essential for sRNA biogenesis (Kanno et al., 2005; Onodera et al., 2005). Whenever NRPD2E2 $2^{\mathrm{Aly}{ }^{\mathrm{MN}} 47}$ is mentioned throughout the text, we refer to the allele $(A L 3 G 37870)$ in the North American $A$. lyrata subsp. Iyrata accession MN47. It is a plant-specific gene with conserved structure of eight exons and seven introns (Figure 14A). Sequence alignment comparison of NRPD2E2 $2^{\mathrm{Aly} \text { MN47 }}$ gDNA and cDNA to its $A$. thaliana ortholog (AT3G23780) NRPD2E2 ${ }^{\text {At_Col }}$ revealed total loss of the seven conserved introns from the retrogene NRPD2E2 $2^{\text {Aly_MN47 }}$ (Appendix E), supporting it's evolutionary origin as a retrogene. However, the published A. lyrata genome annotation had in silico predicted three introns in NRPD2E2 $2^{\text {Aly_MN47 }}$ gene structure (Hu et al., 2011). We further confirmed that by RT-PCR (data not shown) and next generation sequencing (RNA-Seq) (Figure 14D). Mapping the intronic sequences to the orthologous gene in other species has revealed that those introns have emerged through intronization of NRPD2E2 $2^{\text {Aly_MN47 }}$ exonic sequences (Figure 14A). As a result, NRPD2E2 $2^{\mathrm{Aly} \text { MN47 }}$ mRNA transcript was shortened compared to NRPD2E2 ${ }^{\mathrm{At}+\mathrm{Col}}$, although all NRPD2E2 ${ }^{\mathrm{Aly} \_\mathrm{MN} 47}$ exons are represented in the genomic sequence. Consequently, the encoded protein was significantly shorter, which would 
presumably affect protein structure and function.

We encountered another unusual observation when compared NRPD2E2 $2^{\text {Aly_MN47 }}$ gene locus to $A$. thaliana genome (Figure 14C). The retrogene was located in a conserved syntenic block of genes belonging to the parental gene; i.e. the retrogene had the exact genomic position of the parental gene. Interestingly, the parental gene was absent from A. lyrata genome; i.e. was replaced by the retrogene copy in a process called gene targeting. The retrogene copy had reduced gene length coinciding with the length of lost introns. To estimate the approximate age of the event and its conservation in Arabidopsis, we used PCR to compare gene length among $A$. thaliana, Arabidopsis arenosa and several accessions of $A$. lyrata subsp. lyrata and $A$. lyrata subsp. petraea. The results indicate that the event is specific to $A$. lyrata subsp. lyrata accessions (Figure 14B). Hence, it is a relatively recent retroposition event, specific to $A$. lyrata subsp. lyrata, where the retrogene copy has replaced the original (parental) gene copy upon integration and underwent intronization of exonic sequences post-integration. 
A

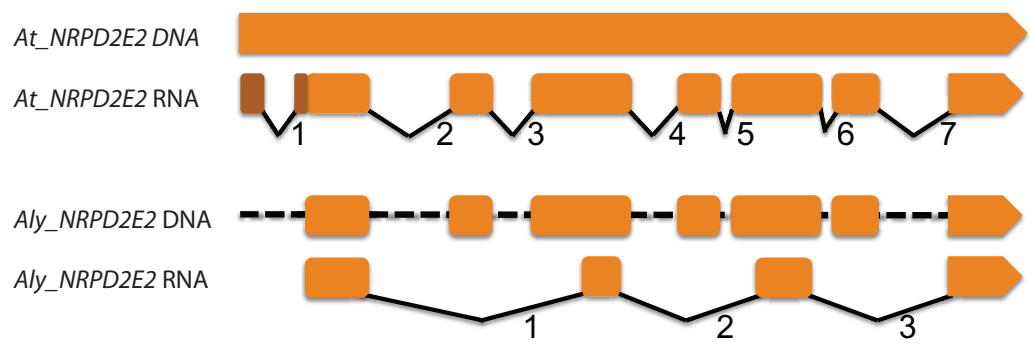

B

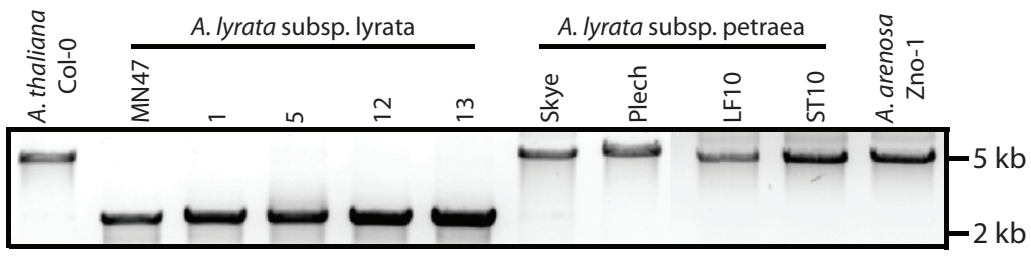

C

A. thaliana, chromosome 3, $8.56-8.59 \mathrm{Mb}$

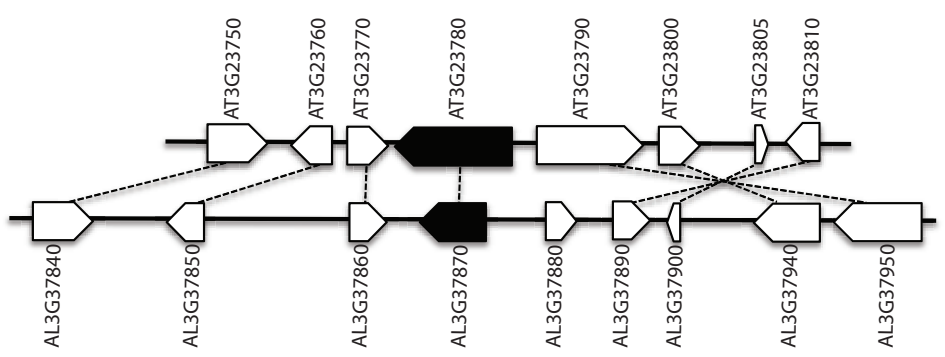

A. lyrata, scaffold 3, $11.06-11.10 \mathrm{Mb}$

D

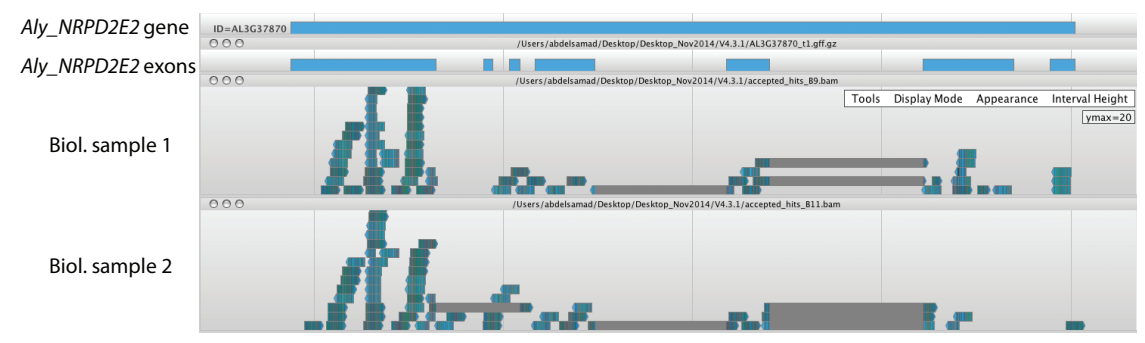

Figure 14. NRPD2E2 ${ }^{\mathrm{Aly} \_\mathrm{MN} 47}$ retrogene targeting event

(A) The seven conserved introns of NRPD2E2 ${ }^{\mathrm{Aly}-\mathrm{MN} 47}$ are lost, causing the gene structure to be similar to the cDNA of $A$. thaliana ortholog $\left(N R P D 2 E 2^{\text {At_Col }}\right)$. Gain of splice sites has led to the intronization of some exonic sequences; and consequently the resulting protein has lost some of its necessary domains. (B) PCR amplification of NRPD2E2 genomic sequence from different Arabidopsis species and accessions, using the same primer pair. It confirms the reduction in NRPD2E2 ${ }^{\mathrm{Aly} \_\mathrm{MN} 47}$ gene length; and suggests that the targeting is a relatively recent event that happened after the speciation of $A$. lyrata ssp. lyrata. (C) Representation of synteny conservation between NRPD2E2 ${ }^{\mathrm{Aly} \_\mathrm{MN} 47}$ and NRPD2E2 $2^{\mathrm{At} \_\mathrm{Col}}$ loci. Conserved synteny location, yet loss of introns indicate retrogene targeting event in NRPD2E2 $2^{\mathrm{Aly} \text { MN47 }}$. (D) Mapped reads of mRNA deep sequencing (RNA-seq) confirm the expression of $N R P D 2 E 2^{A l y}{ }^{\mathrm{MN} 47}$, and confirm the in silico predicted introns shortening its mRNA transcripts. 


\subsection{Arabidopsis retrogene targeting and the origin of their introns}

The NRPD2E2 case of retrogene prompted us to screen the genomes of $A$. thaliana and $A$. lyrata for the frequency of natural retrogene targeting events. To achieve that, we designed a novel bioinformatic tool, Targeted Retrogene Annotation Tool (TRAT) (Figure 15A). The tool runs a genome-wide comparison of protein coding genes between two closely related species to identify retrogene targeting events in both genomes. The gene is considered a primary retrogene targeting candidate in one of the species if has a minimum of three lost introns compared to its syntenic ortholog in the other species. The lost introns should present in both syntenic orthologs in the other species as well as in the out-group. Figure $15 \mathrm{~A}$ depicts the steps and results of a TRAT tool run on $A$. thaliana and A. lyrata genomes. It started by establishing sequence based orthology between 20552 pairs of $A$. thaliana and A. lyrata genes; of which 19694 ortholog pair were located in syntenic blocks, and thus identified as syntenic orthologs and considered for further analysis. Only 473 syntenic orthologous gene pairs had difference of at least four homologous exons spanning three absent introns (363 and 110 in $A$. thaliana and $A$. lyrata, respectively). Then, the gDNA and cDNA of these gene pairs were aligned using MUSCLE v3.8.31 (Edgar, 2004) and visually inspected to evaluate exon sequence homology and intron differences. Unexpectedly, only a single and six cases in A. thaliana and A. lyrata, respectively, have passed our criteria. The high false positive rate was mainly due to discrepancies in annotated intron number per gene. Two main observed scenarios were; first, the same introns were present in both species but annotated as introns in only one of them; second, simple point mutation(s) of splice sites converted intronic sequence in one of the species into exonic or vise versa. The syntenic orthologs of the seven remaining candidate gene pairs were then identified in the out-group genome of $C$. rubella. The ancestral gene structure is crucial to determine whether the difference in intron number among the orthologous genes is a transposition-caused intron loss in one of the species or intron gain in the other. We referred the structure of the ancestral gene from the agreement in structure between the candidate gene in one of the two species and its ortholog in the C. rubella (Figure 15B). Of the seven primary candidates none and two retrogene targeting events were considered as likely true events after manual inspection in $A$. thaliana and $A$. lyrata genomes respectively (Figure $15 \mathrm{C}$ and $\mathrm{D}$ ). 
A

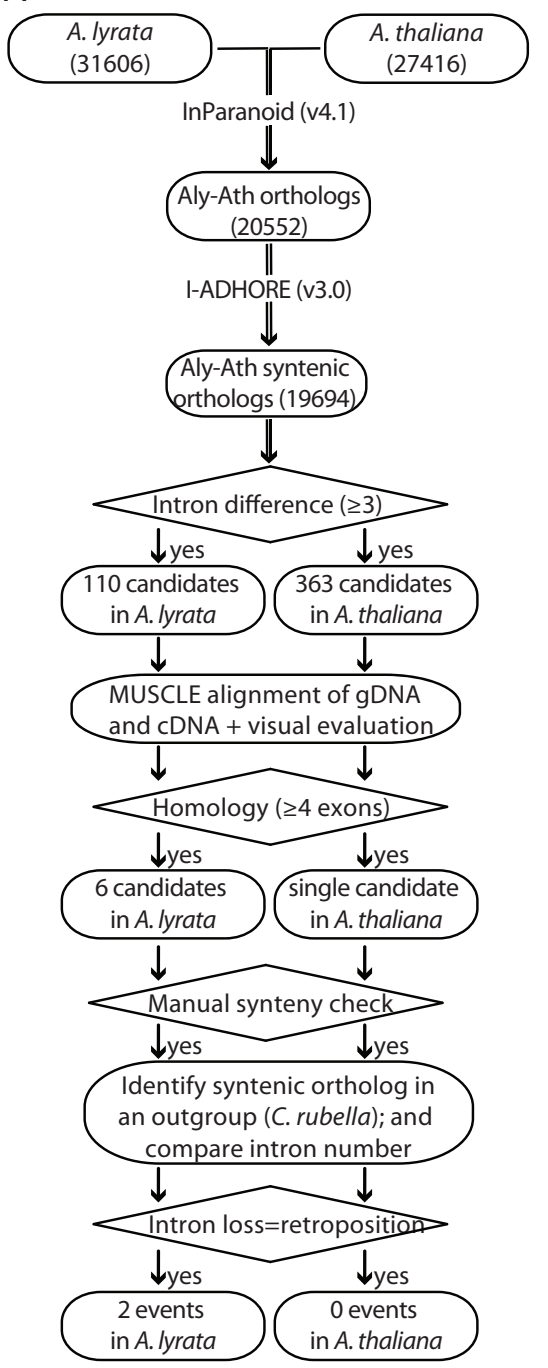

B

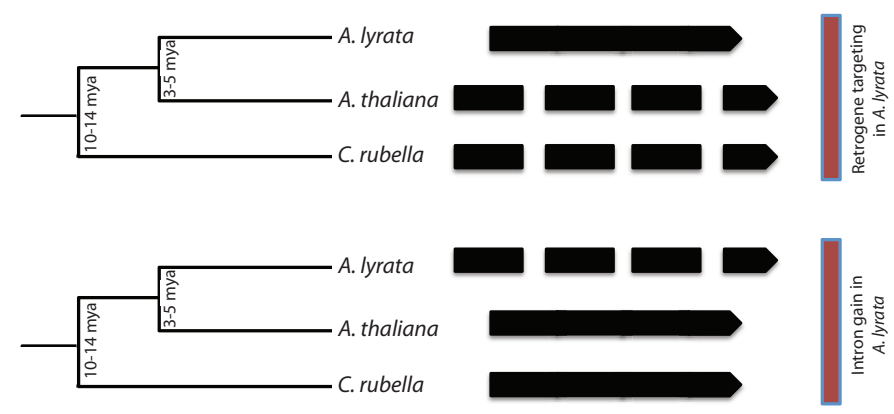

C

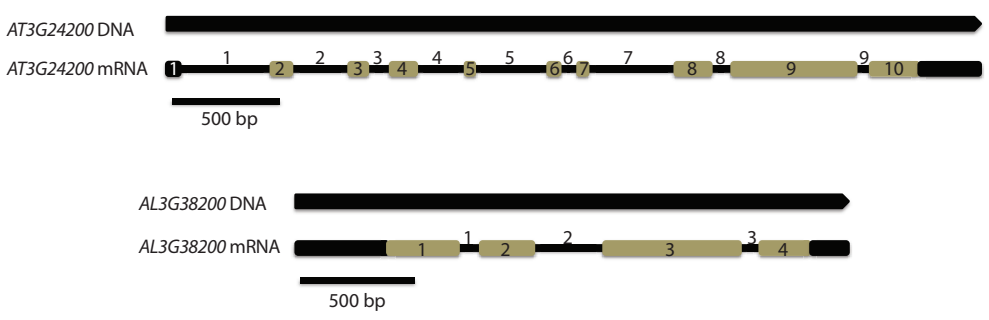

D

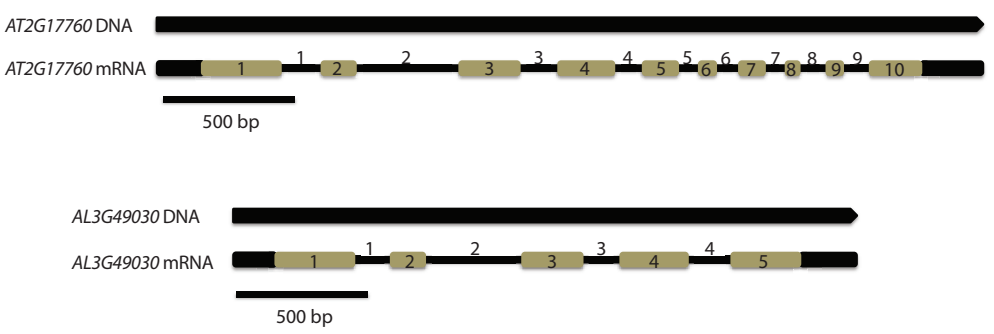

Figure 15. Identification of natural retrogene targeting events using TRAT

(A) Schematic representation of TRAT for identification of retrogene targeting events in Arabidopsis. (B) Schematic comparison of A. thaliana, A. lyrata and C. rubella orthologous gene structure to determine whether the cause of intron number difference is intron gain or transposition-based intron loss. (C, D) Gene models of the A. lyrata retrogene-targeting events and the $A$. thaliana orthologs of their parental genes.

Table 5. Natural retrogene targeting events

\begin{tabular}{|c|c|c|c|c|c|c|c|c|}
\hline $\begin{array}{l}\text { A. thaliana } \\
\text { ortholog }\end{array}$ & 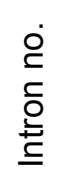 & $\begin{array}{l}\text { Gene } \\
\text { /CDS } \\
\text { length }\end{array}$ & $\begin{array}{l}\text { A. lyrata } \\
\text { ortholog }\end{array}$ & $\begin{array}{l}\dot{0} \\
\stackrel{2}{c} \\
\text { Oे } \\
\text { 을 }\end{array}$ & $\begin{array}{l}\text { Gene } \\
\text { /CDS } \\
\text { length }\end{array}$ & $\begin{array}{l}\text { C. rubella } \\
\text { ortholog }\end{array}$ & 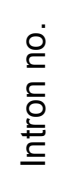 & $\begin{array}{l}\text { Gene } \\
\text { length }\end{array}$ \\
\hline $\begin{array}{c}\text { AT3G24200 } \\
\text { monooxygenase }\end{array}$ & 9 & $\begin{array}{c}3.8 \\
11.5 \mathrm{~kb}\end{array}$ & AL3G38200 & 3 & $\begin{array}{c}2.4 \\
/ 1.3 \mathrm{~kb}\end{array}$ & Carubv10015259m & 9 & $\begin{array}{c}3.4 \\
/ 1.5 \mathrm{~kb}\end{array}$ \\
\hline $\begin{array}{c}\text { AT2G17760 } \\
\text { aspartyl protease }\end{array}$ & 9 & $\begin{array}{c}3.2 \\
11.2 \mathrm{~kb}\end{array}$ & AL3G49030 & 4 & $\begin{array}{c}2.4 \\
/ 1.2 \mathrm{~kb}\end{array}$ & Carubv10016111m & 9 & $\begin{array}{c}2.8 \\
/ 1.5 \mathrm{~kb}\end{array}$ \\
\hline
\end{tabular}


The two A. lyrata targeted retrogenes were AL3G38200 and AL3G49030 (Table 5). The first targeted retrogene (AL3G38200) was the syntenic ortholog of $A$. thaliana monooxygenase (AT3G24200). It was localized in the exact syntenic position of its parental gene; which in turn was absent from the genome. The gene encodes a conserved $F A D / N A D(P)$-binding oxidoreductase family protein found from yeast to humans. Comparing gene structure in A. thaliana, C. rubella and Camelina sativa showed a relatively conserved structure of ten exons and nine introns in Brassicaceae (Figure 15C). However, gDNA and CDNA sequence alignment revealed the loss of eight of the nine conserved introns in the allele of $A$. lyrata subsp. lyrata; i.e. one parental intron was retained in the precursor mRNA or acquired postintegration (Table 6). The loss of introns coincided with the reduction in gene length in A. lyrata compared to relatively conserved cDNA length (Table 5). However, our RNAseq-supported annotation of $A$. lyrata genome has predicted two more introns in gene structure; in addition to the retained parental intron (Figure 15C). When analyzed, the two introns showed no significant homology to the syntenic orthologs or any of $A$. thaliana or $C$. rubella sequences, but had a minimum of $94 \%$ sequence identity to multiple $A$. lyrata-specific intergenic regions. This suggests that the integration of these A. lyrata-specific introns in the targeted retrogene was relatively recent and happened post integration of the nascent retrogene copy (Table 6).

The second targeted retrogene (AL3G49030) was the syntenic ortholog of $A$. thaliana (AT2G17760). It was localized in the conserved syntenic position of its parental gene; which in turn was absent from the genome. The gene encodes a relatively conserved aspartyl protease family protein. Comparing gene structure in $A$. thaliana, C. rubella, C. sativa, Brassica rapa and Eutrema salsugineum showed a well-conserved structure of ten exons and nine introns in Brassicaceae (Figure 15D). However, gDNA and cDNA sequence alignment revealed the loss of six of the nine conserved introns in A. lyrata allele; i.e. one parental intron was retained in the precursor mRNA or acquired post-integration. This was supported by the high sequence identity between the three retained introns and the syntenic ortholog introns (Table 6). The loss of introns coincided with a reduction in gene length in $A$. lyrata compared to relatively conserved cDNA length (Table 5). Our RNAseqsupported annotation of $A$. lyrata genome has predicted a fourth intron in gene structure (Figure 15D). When analyzed, the intron did not show homology to $A$. thaliana syntenic ortholog; but showed homology to many other aspartyl protease 
family proteins. This suggested the retention of this intron from the parental precursor mRNA or post integration acquisition (Table 6).

Table 6. Origin of targeted retrogenes introns

\begin{tabular}{cccccc}
\hline Intron & Coordinates & Length & Parental? & Possible origin & Note \\
\hline & & & AL3G38200 & & \\
\hline $1^{\text {st }}$ & $11298541-11298640$ & $100 \mathrm{nt}$ & No & Post-integration & Intergenic in A. Iyrata \\
$2^{\text {nd }} \mathrm{A}$ & $11298875-11299100$ & $226 \mathrm{nt}$ & No & Post-integration & Intergenic in A. Iyrata \\
$2^{\text {nd }} \mathrm{B}$ & $11299101-11299179$ & $79 \mathrm{nt}$ & Yes $(100,90)^{*}$ & Intron retention & - \\
$3^{\text {rd }}$ & $11299773-11299856$ & $84 \mathrm{nt}$ & Yes $(100,84)^{*}$ & Intron retention & - \\
\hline & & & $A L 3 G 49030$ & & \\
\hline $1^{\text {st }}$ & $22236470-22236619$ & $150 \mathrm{nt}$ & Yes $(100,90)^{*}$ & Intron retention & Family specific intron \\
$2^{\text {nd }}$ & $22236755-22237127$ & $373 \mathrm{nt}$ & Yes $(97,91)^{*}$ & Intron retention & Family specific intron \\
$3^{\text {rd }}$ & $22237364-22237505$ & $142 \mathrm{nt}$ & Yes $(100,81)^{*}$ & Intron retention & Family specific intron \\
$4^{\text {th }}$ & $22237774-22237922$ & $149 \mathrm{nt}$ & No & Post-integration & Family specific intron \\
\hline
\end{tabular}

${ }^{*}$ Intron coverage $\%$ and identity $\%(C, \mathrm{I})$ to parental intron, respectively.

\subsection{Introns increase stability of retrogenes transcripts}

Most retrogene copies are expected to be intron-less at the time of integration. However, the three identified targeted retrogenes and approximately one-third of Arabidopsis retrogenes contained introns, many of which were acquired post integration. This indicated that retrogene intronization has a functional role. We tested whether intronization plays a role in retrogene mRNA stability. First, we compared the mRNA half-life of transcribed retrogenes in $A$. thaliana $(n=100)$, parents $(n=147)$ and the genome-wide set of transcribed genes $(n=13,012)$ included in the publicly available mRNA decay dataset (Narsai et al., 2007). The mRNA half-life of the parents and the genome-wide gene set was similar (MWW test, $P=0.21$ ) and significantly longer than that of the retrogene mRNA (MWW test, $P=$ $3.56 \times 10^{-5}$ and $2.54 \times 10^{-5}$, respectively; Figure 16A). Furthermore, mRNA of introncontaining retrogenes $(29 \%)$ had a slightly but significantly longer half-life compared to that of intron-less retrogenes (MWW test, $P=0.04$; Figure 16B). Hence, acquisition of introns increases retrogene mRNA half-life. 

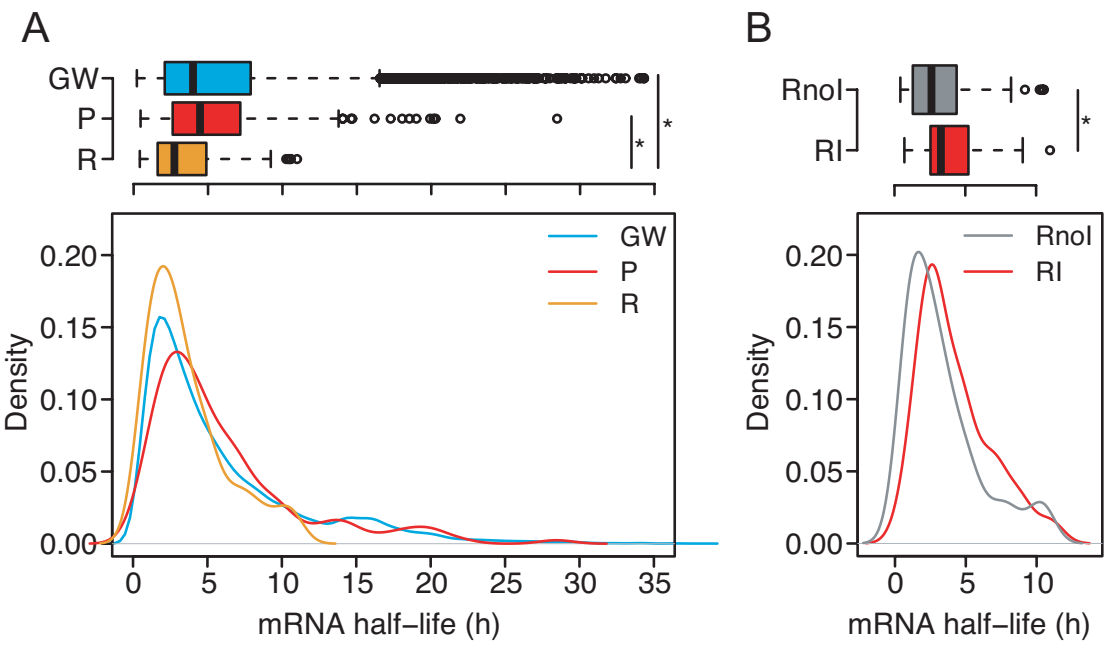

Figure 16. Introns increase half-life of retrogenes transcripts

(A, B) mRNA half-lives of genome-wide genes (GW), parents $(P)$, retrogenes $(R)$, intron-less retrogenes $(\mathrm{Rnol})$ and intronized retrogenes $(\mathrm{RI})$. Significances were calculated using the Mann-Whitney-Wilcoxon test for all group combinations within each graph, and asterisk in box plots indicate significant differences $(P<0.05)$. Non-significant $(P \geq 0.05)$ relationships are not shown. 


\section{DISCUSSION}

\subsection{The novel bioinformatic retrogene annotation tool (RAT) proved useful for identification of retrogenes across plant genomes}

Gene duplication is a major force in genome evolution and adaptation. RNAmediated gene duplication (retroposition) is capable of generating evolutionary innovations and providing fast responses to environmental challenges at single gene level. Single gene duplications have been also linked to human diseases including Parkinson's and cancer (Chartier-Harlin et al., 2004; Cooke et al., 2014) RNAmediated duplicated genes (retrogenes) are reverse-transcribed from mature mRNA transcripts of protein coding genes (parent genes) and integrate randomly in the genome. Therefore, retrogenes are usually intron-less, retropose without their regulatory sequences and might have a poly $(\mathrm{A})$-tail in their downstream regions. We used these retrogene-specific characters to annotate them in Arabidopsis genomes. We developed a novel bioinformatic Retrogene Annotation Tool (RAT) to screen the genomes of $A$. thaliana and $A$. lyrata for retrogenes (Figure $1 \mathrm{~A}$ and $11 \mathrm{~B}$ ); and consequently studied their genomic features, expression, epigenetic regulation and evolution.

\subsubsection{Annotation of retrogenes in $A$. thaliana genome (TAIR10)}

We annotated 251 retrogenes in $A$. thaliana genome (Appendix A), 216 of which had not previously been identified (Zhang et al., 2005; Zhu et al., 2009). The limited overlap of our set with the previous $A$. thaliana retrogene lists was most likely due to partly different search criteria and thresholds of individual methods. We detected approximately $50 \%$ of the retrogenes found in the study of Zhang et al., (2005). A specific subset of the remaining retrogenes was not accepted by RAT tool, owing to different thresholds for selection or lack of positive evidence for retroposition such as missing information on parental gene or insufficient difference in introns number. The smaller (43.2\%) overlap with the set identified by Zhu et al. (2009) is due to their use of very specific criteria to identify chimeric retrogenes. These criteria apparently hamper identification of structurally simple retrogenes; while, conversely, 
RAT tool does not allow identification of chimeric retrogenes. The higher number of retrogenes detected with our analysis is most likely due to several factors: (i) search among $A$. thaliana pseudogenes; (ii) allowing intronized retrogenes; and (iii) accepting multiple retrocopies derived from a single parent (applied also in Zhang et al., 2005). Although we increased the number of retrogenes in $A$. thaliana three-fold, our selection criteria were conservative and the current number is most likely an underestimate based on two facts. First, we omitted several hundred candidates that had at least one paralog within the $A$. thaliana genome but did not show evidence of retroposition (i.e. did not differ by $\geq 2$ introns nor had a polyA-tail). Second, none of the plant genome-wide retrogene screens detected retrogenes of the SET-domain protein group (Abdelsamad and Pecinka, 2014; Zhang et al., 2005; Zhu et al., 2009), which were identified in studies focusing specifically on the evolution of this gene family (Baumbusch et al., 2001; Borg et al., 2011; Zhu et al., 2011). Hence, $1 \%$ of $A$. thaliana genes estimated to be retrogenes is most likely an underestimation.

\subsubsection{Identification of $A$. lyrata retrogenes using the newly developed genome annotation}

The close taxonomic relationship of $A$. lyrata to $A$. thaliana and the recent release of its genome sequence made it a promising model organism to study gene and genome evolution in comparative studies (Clauss and Koch, 2006; Hu et al., 2011). Northern rock-cress, $A$. lyrata, is a perennial outcrosser that has two main subspecies; the eurasian $A$. lyrata subsp. petraea and the North American A. lyrata subsp. lyrata. The sequenced accession, MN47, belongs to the latter subspecies, and is referred to throughout this thesis (Clauss and Koch, 2006; Hu et al., 2011). The published gene models of $A$. lyrata genome were almost solely based on in silico prediction tools. Although usually sufficient for general annotation of genic versus intergenic regions, but incomprehensive annotation of exon-intron boundaries and alternative splicing isoforms hinders any genome-wide search for retrogenes. Therefore, an enhanced version of $A$. lyrata gene models annotation, developed by a collaboration of several laboratories including my input, was a prerequisite for successful retrogene identification. The work is currently submitted for publication. Using this resource in combination with our novel bioinformatic RAT, we revealed the first of retrogenes in $A$. lyrata genome. In total we identified 168 retrogenes passing 
stringent selection criteria; representing $0.53 \%$ of $A$. lyrata protein coding genes.

Although we have found approximately double and three-fold more retrogenes in $A$. lyrata and $A$. thaliana, respectively, than previously found in rice (Sakai et al., 2011), the number of conservatively estimated retrogenes per plant genome is much smaller compared to metazoans, e.g. $19.1 \%$ in humans (Marques et al., 2005; Pennisi, 2012). This difference may have several reasons. Since most of the retrogenes are identified based on intron loss, greater intron numbers in metazoan parents would simplify retrogene identification. This may partially explain the difference between $A$. thaliana and human genomes, which have average numbers of 4.2 and 7.8 introns per gene, respectively (Arabidopsis Genome Initiative, 2000; Sakharkar et al., 2004). Another possibility, which is not mutually exclusive, builds on the scarcity of WGDs in many groups of higher animals compared to plants (Gregory and Mable, 2005). This may favor local gene duplication mechanisms, including retroposition, in metazoa versus plants. Finally, higher activity of LINE element reverse transcriptases may be responsible for an increased retroposition rate in animals (Beck et al., 2010).

In contrast to animals, where $82 \%$ of retrocopies contain premature stop codons (Marques et al., 2005), only $17.4 \%$ of $A$. thaliana retrogenes are annotated as pseudogenes. This suggests a higher retrogene success rate in plants relative to the total number of retrocopies (Abdelsamad and Pecinka, 2014). Further support comes from our observation that several retrogenes served as parents and produced secondary retrocopies. Therefore, retroposition contributes to the functional plant genome evolution.

\subsection{Fast evolutionary emergence of Arabidopsis retrogenes}

Retroposition-based gene duplication contributes to plant genome evolution. To evaluate retrogene role in Arabidopsis genome evolution, we aimed to calculate their evolutionary emergence rate through comparing their conservation in $A$. thaliana and $A$. lyrata genomes, with $C$. rubella as an out-group. Gene duplication may elevate the selection pressure put on the parent gene; allowing the parent and/or the retrogene to accumulate DNA sequence polymorphisms, possibly leading to their sub- or neofunctionalization. This is an evolutionary advantage; however, from a technical point of view, post-transposition sequence polymorphisms represent a 
challenge towards retrogene identification screens and orthology searches. We established orthology among $A$. thaliana, A. lyrata and C. rubella protein coding genes to study retrogene conservation among them. About $86 \%$ (145) of the identified $A$. lyrata functional retrogenes $(n=168)$ were conserved genes in $A$. thaliana and/or $C$. rubella (Figure 11C). Similarly, $75 \%$ (157) of $A$. thaliana functional retrogenes, identified solely by our RAT tool $(n=208)$, were conserved genes in $A$. lyrata and/or $C$. rubella. This strongly argues for the retroposition preceding the split of $A$. thaliana and $A$. lyrata lineages, about 3-5 million year ago. Nevertheless, only small number of these pre-split retrogenes (61) is conserved as retrogenes in both species. The rest of retrogenes had orthologous genes in the other species that were not identified as retrogenes by RAT tool. A possible reason is that during the evolutionary past of these retrogenes, they might have lost retrogene-specific features in one of the species; and thus they didn't pass our stringent selection criteria. The average half-life of eukaryotic duplicated gene is 4.0 million years (Lynch and Conery, 2003); however, the existence of orthologs for many of the identifed retrogenes in C. rubella genome indicates a longer half-life, which is often associated to neofunctionalization (Konrad et al., 2011).

The remaining $14 \%(23)$ and $25 \%$ (51) of $A$. lyrata and $A$. thaliana functional retrogenes, respectively, were species-specific; i.e. have transposed after the split of the two species from the last common ancestor 3-5 million years ago. An alternative explanation of species-specific retrogenes would be the loss of the orthologs from the other species. However, we couldn't find the orthologs of these retrogenes in the outgroup species as well. This supports the hypothesis of post-split retroposition in one of the species rather than loss of the orthologous retrogene from the other. This hypothesis is further supported by significantly higher nucleotide similarity between species-specific retrogenes and their parental genes in $A$. thaliana and $A$. lyrata (Figure 11). With 23 and 51 post-split transposition events in $A$. lyrata and $A$. thaliana, respectively, we calculated the evolutionary rate of retrogene emergence in A. thaliana genome. With 5 MYA as the high limit of divergence between the two species (Clauss and Koch, 2006), we estimated a minimal rate of 5-10 successful retroposition events per one million of years. That is at least ten times faster rate than previously calculated for Arabidopsis retrogene duplication (Zhang et al., 2005). Our stringent criteria used for retrogene identification have excluded many false negative 
events that would have even further increased the estimated evolutionary rate of retrogene emergence.

In general, retrogene identification is a complex process, and possibly all retrogene identification methods suffer from a specific false positive discovery rate, that is currently difficult to estimate. Additionally, retrogene annotation methods are faced by multiple challenges that increase their false negatives. Three of these challenges are; 1) post-retroposition evolution of retrogenes and their parental genes causes the loss of retrogene specific features, hindering their identification; 2) alternative and trans-splicing of the precursor mRNA may result in chimeric retrogenes which do not match standard selection criteria; 3) the quality of genome annotation is a limiting factor in retrogene identification. Consequently, many true retrogenes might get excluded due to the lack of positive evidence.

\subsection{Multiple and repeated retropositions in Arabidopsis}

One of the unresolved questions in retrogene biology is how transcripts are selected for retroposition. Although retroposition in animals has been associated with LINE element amplification machinery, this link has not been firmly proven in plants (Ohshima, 2013). Our data show that parent genes gave rise to multiple retrogenes before and after the split of $A$. thaliana and $A$. lyrata from the last common ancestor; and some underwent secondary retropositions. We describe twenty-two parents that produced up to seven retrogenes each in $A$. thaliana. Similarly, six parents produced up to four retrogene each in $A$. lyrata. This indicates non-random selection of parental mRNA for retroposition at least in some cases. The highly non-random pattern strongly suggests one or more common features or a signal for retroposition in Arabidopsis. Another support for non-random selection of precursor transcripts comes from the six and thirteen cases where a repeated retroposition has been found in $A$. lyrata and $A$. thaliana respectively (Table 1 and 5 ). Repeated retroposition occurs when the mRNA transcript of a retrogene serves as a precursor for a secondary retroposition. Since retrotransposon reverse transcriptases favor specific sequences in combination with transcript folding (Ohshima, 2013), it is possible that such structures exist also in transcripts of some protein coding genes. However, our preliminary efforts to identify the most common sequence motifs among those parents as potential signal for transposition were inconclusive. Therefore, among our 
planned future analyses is the search for potential transposition signals in the primary sequence and the secondary structure of parent transcripts as suggested by in silico tools (Ohshima, 2013). Similarly to other plant and animal studies (Marques et al., 2005; Potrzebowski et al., 2008; Sakai et al., 2011), we have confirmed that parents are generally strongly and ubiquitously transcribed (Figure $3 \mathrm{~A}$ and $13 \mathrm{C}$ ), indicating that higher amounts of transcript may increase the probability of retroposition. Although produced by the retrotransposon amplification machinery, retrogenes are located in gene rich chromosome arms (euchromatin) in $A$. thaliana (Figure 2) and $A$. lyrata,(Figure 12) and thus fundamentally differ in their genomic distribution from repetitive elements. This also holds true for their up- and down-stream intergenic regions that are not enriched for repetitive DNA (Figure 3A).

Hence, multiple and repeated retroposition indicate non-random selection of retrogene precursor transcripts from strongly and ubiquitously transcribed genes. And retrogenes integrate in gene-rich regions (open chromatin), and that facilitates their transcription to meet cellular requirements. However, they usually lack promoter sequences, as they are reverse transcribed from mature mRNA transcripts.

\subsection{Arabidopsis retrogenes are transcribed via newly acquired promoters}

One of the major limitations to the establishment of retrogenes as functional genes is the loss of cis-regulatory sequences (Kaessmann et al., 2009). Therefore, transposed retrocopies that cannot acquire regulatory sequences to be expressed often accumulate mutations and turn into processed pseudogenes (Hirotsune et al., 2003; Pink et al., 2011). Hence, we analyzed the retrogene transcription in $A$. thaliana using genome-wide transcription data of 49 different $A$. thaliana developmental stages by microarrays. In agreement with the observations in rice (Sakai et al., 2011), we found that retrogenes are transcribed less compared to their parents (Figure $3 \mathrm{~A}$ ). However, retrogene transcription resembles the whole genome average, suggesting that they are not 'dead on arrival' in $A$. thaliana. We saw similar pattern for $A$. lyrata retrogenes using next generation sequencing (RNA-seq) data (Figure 13C).

In humans, it has been shown that retrogenes and parents may share promoter sequences, implying a carry-over of the parental promoter by retroposition of transcripts from an upstream TSS (Okamura and Nakai, 2008). Furthermore, a 
recent study in rice revealed a number of retrogene-parent pairs with positively correlated transcription profiles among seven developmental stages (Sakai et al., 2011). However, this analysis did not include correction for co-transcription of random gene pairs (Sakai et al., 2011) and therefore the extent of correlation may be partially overestimated. Our data show that approximately $25 \%$ of retrogene-parent pairs and $3 \%$ of retrogene head-to-head oriented neighboring genes are co-transcribed beyond genome background in $A$. thaliana. Hence, rice and Arabidopsis data support the mechanism of cis-regulatory element carry-over in plants. However, DNA sequence analysis of parent and retrogene promoters did not reveal significant homology in rice (Sakai et al., 2011). We show similar results for $A$. thaliana (Figure 3E). Therefore, it remains unclear whether retrogenes retropose including parental upstream regulatory sequences that mutate rapidly afterwards, or they carry cryptic exonic regulatory sequences. In $A$. thaliana, majority (72\%) of retrogenes are transcribed in a pattern that is not correlated to that of parents and neighboring genes, suggesting acquisition of novel cis-regulatory elements in most cases. Currently it is unknown whether this pattern is the result of post-integration selection or whether the compact $A$. thaliana genome offers a sufficient density of cryptic promoters.

\subsection{Retrogenes are preferentially up regulated in pollen}

In flies and mammals, many retrogenes are transcribed specifically in male germ cells (Bai et al., 2008; Marques et al., 2005; Vinckenbosch et al., 2006). The separation of gametes from somatic cells is very much delayed in plant compared to animal development (Wang and Ma, 2011). Therefore, somatic retroposition events in the shoot apical meristems may also be transmitted to the next generations. Therefore, we tested for tissue specific transcription of retrogenes in $A$. thaliana using a developmental transcription data series (Schmid et al., 2005) and validated our findings using RNA-sequencing datasets (Loraine et al., 2013). Surprisingly, this revealed that retrogenes are over-transcribed in pollen while overall transcription was not increased at this stage (Figure 4A). However, the pollen-specific up-regulation of retrogenes was not uniform for the whole group, as lowly transcribed retrogenes became up regulated in pollen while highly-transcribed ones were down-regulated. In addition, the set of all $A$. thaliana genes showed a similar trend. Hence, this transcription pattern is not restricted to retrogenes. More likely, many retrogenes are 
part of global cellular reprogramming in male gametes. So far, chromatin changes in male gametes have been associated mainly with DNA methylation changes (Ibarra et al., 2012; Slotkin et al., 2009), but there is emerging evidence that histone modifications may also contribute to pollen-specific gene reprogramming (Borges et al., 2012; Hoffmann and Palmgren, 2013). In order to identify possible causes of the observed pollen-specific transcription, we explored available data on tissue- and mutant-specific transcription and distribution of chromatin modifications. By comparing transcriptional profiles of pollen and mutants defective in transcriptional gene silencing, we excluded loss of DNA methylation and repressive H3K9me2 or heterochromatin-specific histone hyper-acetylation as the factors leading to global transcription changes in pollen. The analysis of chromatin profiles in leaves revealed that pollen up-regulated genes (and retrogenes) are depleted of transcription permissive marks (H2Bub, H3K4me3 and H3K36me3) in these tissues. Recently, it has been reported that pollen-specific genes are controlled by H3K27 methylation in Arabidopsis (Hoffmann and Palmgren, 2013), but this trend was much less pronounced in our dataset. This is due to different selection criteria of candidate genes in both studies. Our set of pollen up-regulated genes $(n=5,171)$ included the entire (99.1\%) set of pollen-specific genes ( $n=584$; Hoffmann and Palmgren, 2013). This is most likely masking the enrichment for H3K27me modifications of specificsubset of pollen-transcribed genes in leaves. However, it has to be noted that H3K27me3 modification may control pollen-specific transcription indirectly, as suggested by our transcription analysis of the CLFISWN and FIE mutants. This also holds true for the group of pollen-specific genes associated with H3K27me1 and me3 in leaf tissues (Hoffmann and Palmgren, 2013), as only a few of those genes are upregulated in clf/swn (Figure 8D). Unexpectedly, we found correlated down-regulation of similar sets of genes (and retrogenes) in pollen and leaves of clf/swn or fie ( $r=$ 0.462 and 0.366 , respectively). This indicates down-regulation of genes (and retrogenes) in response to lack of repressive chromatin marks in mutants of the polycomb group repressive complex factors. Gene down-regulation in response to the loss of repressive mark is counterintuitive and suggests that the effect is indirect, and may be achieved by an activation of specific H3K27me3 controlled suppressors such as miRNAs (Lafos et al., 2011). Based on this, we suggest that it is most likely temporary absence of permissive marks (without strong enrichment for repressive 
marks) that causes up-regulation of specific genes in pollen relative to somatic tissues.

Pollen-specific transcription of $A$. thaliana retrogenes was unanticipated and is analogous to retrogene transcription in animal spermatocytes (Marques et al., 2005; Vinckenbosch et al., 2006; Bai et al., 2008). Although the molecular nature of this specific transcription is so far unknown, two explanatory models have been proposed in animals (Kaessmann et al., 2009). The first suggests sperm-specific retroposition and integration into open (and thus more likely to be transcribed) chromatin that allows transcription and perpetuates this behavior. However, our data do not support this model in two aspects. First, integration into active chromatin would most likely be reflected by co-transcription between neighboring genes, which was rare in $A$. thaliana. Second, we observed many non-retrogene-genes with pollen-specific transcription. The second model proposes spermatocyte-specific transcriptional reprogramming by global chromatin changes and transcriptional activation of retrogenes and their subsequent functionalization specific to spermatocytes (Marques et al., 2005; Potrzebowski et al., 2008). In plants, pollen have been identified as the hot spot of chromatin reprogramming (Slotkin et al., 2009; Ibarra et al., 2012; Hoffmann and Palmgren, 2013), and we have shown that pollen upregulated genes are depleted from transcription permissive chromatin marks in somatic tissues. Furthermore, we found several retrogenes that are associated with pollen growth and development and the PCR11 retrogene that is transcribed in pollen, contrary to its parent. This is due to the presence of multiple pollen-specific DUO1 transcription factor binding motifs in its promoter. Hence, our data support the second model, and suggest that a small number of retrogenes has developed or retained male gamete-specific functions in $A$. thaliana.

The activation of many normally lowly transcribed genes and subsequent down-regulation of highly transcribed genes just prior to the onset of the next generation is an intriguing pattern with no known molecular function. However, it seems to be present in both plant and animal lineages and suggests evolutionarily conserved or analogous mechanisms that control gene transcription during this critical stage of development. 


\subsection{Arabidopsis retrogenes and retrotransposons share retroposition mechanism but not transcriptional regulation.}

In mammals, LINE1 (long interspersed nuclear element 1 ) is a very active retrotransposon that reverse transcribes precursor transcripts of cytosolic mRNA molecules, generating retrogenes (Ding et al., 2006). LINEs have been proposed to catalyze retrogene transposition in plants; however, this has not been experimentally supported so far (Ohshima, 2013). On average, retrogenes have significantly higher transcription levels than that of TEs (Figure 13C), suggesting a different regulatory mechanism of retrogenes and TEs. Plant cells use regulatory small RNA (sRNA) to orchestrate the transcription levels of genes and TEs (Chen, 2009). Transposition and duplication of TEs signal the cell to exert a tight epigenetic transcriptional silencing preferentially through targeting by $24 \mathrm{nt}$ sRNAs. In contrast, $21 \mathrm{nt}$ sRNA molecules preferentially orchestrate transcription of protein-coding genes (Creasey et al., 2014; Slotkin et al., 2009). We found that the pool of sRNA targeting retrogenes is enriched for gene-specific $21 \mathrm{nt}$ sRNAs and depleted of TE-specific $24 \mathrm{nt}$ sRNAs, similar to other genes genome wide and in contrast to DNA and retrotransposons. Additionally, parental genes show significantly higher transcription levels than other genes in the genome, which might explain the high level of 21nt sRNA targeting (Figure 13C, E). This also suggests that those $21 \mathrm{nt}$ sRNA are rather regulatory miRNAs. Hence, retrogenes share the same machinery of TEs, yet expressed and regulated at different pattern, which mirrors that of other genes.

\subsection{Arabidopsis natural in planta retrogene targeting}

During our manual inspection of conserved retrogenes between $A$. thaliana and $A$. lyrata, we identified NRPD2E2 $2^{\mathrm{Aly}-\mathrm{MN} 47}$ as retrogene targeting event in A. lyrata genome. NRPD2E2 is an indispensable component of small RNA biogenesis and transcriptional gene silencing in A. thaliana (Kanno et al., 2005; Onodera et al., 2005; Ream et al., 2009). The gene itself has emerged as a retrogene at the onset of land plant evolution (Tucker et al., 2010). After the split of $A$. thaliana and A. Iyrata, 3-5 million years ago, another event of retroposition has occurred, where another cDNA copy of NRPD2E2 was generated in A. lyrata subsp. lyrata (Figure 17). However, the generated copy didn't integrate in the genome randomly as most retrogenes; but 
instead replaced the original (parental) gene copy. Although there is no direct experimental evidence for this model, it is strongly supported by the fact that $N R P D 2 E 2^{A l y-M N 47}$ is in the exact syntenic position to its $A$. thaliana ortholog (NRPD2E2 $2^{\mathrm{At}-\mathrm{Col}}$ ); yet the structure and sequences of its genomic DNA matches the CDNA structure of NRPD2E2 At-Col (Figure 14). The targeting event is specific to accessions of $A$. lyrata subsp. lyrata, where changes in DNA sequence has created functional splice sites leading to intronization of exonic sequences, which resulted in shorter mature transcript and protein sequence. To our knowledge, this is the first reported case of in planta gene targeting.

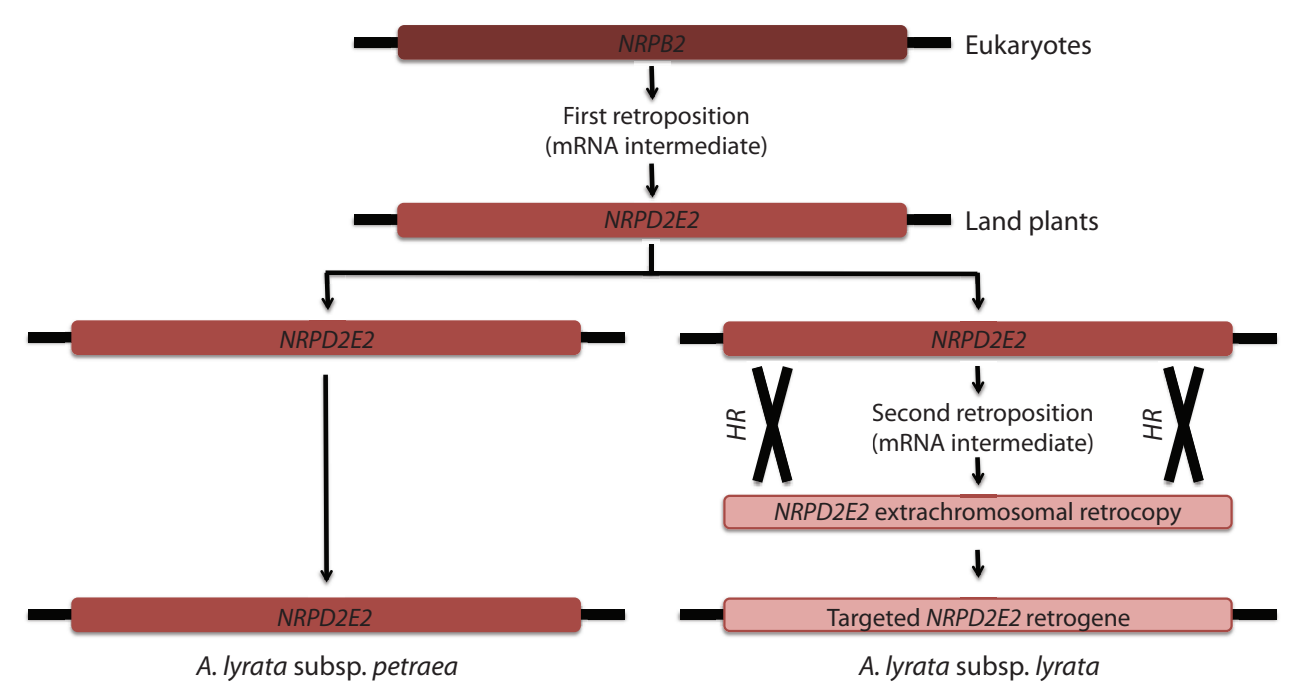

Figure 17. Evolution of NRPD2E2 gene in A. lyrata.

NRPD2E2 emerged as a retrogene from the second largest subunit of RNA polymerase II (NRPB2) at the onset of plant evolution. The gene was replaced, through a homologous recombination (HR), with a retrocpoy generated by a second retroposition event in A. lyrata subsp. lyrata.

Gene targeting is a genetic process that requires homologous recombination to exchange two genetic elements with adequate sequence homology (Ishizaki et al., 2013). Large scale retrogene targeting has been reported in the yeast Saccharomyces cerevisiae, where targeted integration of retrogenes replace the original parent genes leaving intron-less copies in the exact chromosomal loci (Fink, 1987). Our results show that Arabidopsis retrogenes often integrate in gene-rich regions independently of the position of their parental genes. However, for retrogene targeting events, the absence of the parent gene hinders the identification of targeted retrogenes in screens that depend on intraspecies parent-retrogene paralogy; e.g. 
RAT tool. Therefore, we designed a novel bioinformatic Targeted Retrogene Annotation Tool (TRAT tool) to screen for retrogene targeting events in Arabidopsis genomes. TRAT tool identified zero targeted retrogenes in $A$. thaliana and only two retrogene targeting events in $A$. lyrata, not including. NRPD2E2 ${ }^{\text {Aly-MN47 }}$. It was not

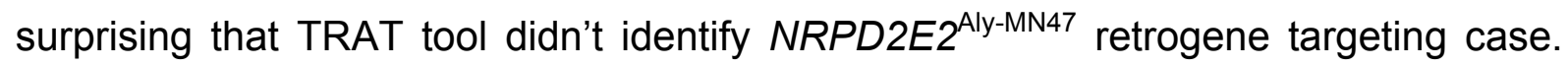
The intronization of NRPD2E2 exonic regions has significantly altered protein sequence. That in turn, hindered establishing protein sequence based orthology between $A$. thaliana and A. lyrata NRPD2E2 orthologs, a crucial step in TRAT tool to define retrogene targeting events.

In total, we identified three targeted retrogenes in A. lyrata genome and none in A. thaliana. Based on our calculation, retrogenes are generated at a rate of five to ten events per million year per species. Then the machinery of homologous recombination selects targeting candidates out of this pool. Unlike in yeast and mammals, homologous recombination is a minor DNA repair pathway in plants, causing gene-targeting rate of $\left(\sim 3 \times 10^{-6}\right)$ in Arabidopsis (Jelesko et al., 1999). Therefore, the low rate of retroposition and homologous recombination in Arabidopsis explain the very little numbers of targeting events in Arabidopsis.

Both targeted retrogenes identified by TRAT were functional and transcribed in our analyzed tissues, indicating that targeting has not affected gene transcription. Most of retrogenes are expected to be intron-less at the time of integration; however, considerable number of retrogenes found in this study contained introns, including the targeted retrogenes (Table 5). There are multiple described mechanisms of intron gain for genes and retrogenes (Fablet et al., 2009; Irimia et al., 2008; Roy and Irimia, 2009; Szczesniak et al., 2011; Yenerall et al., 2011). This includes; Intron Transfer, in which an intron of a paralog is transferred to an intron-absent position in the other paralog; Tandem Genomic Duplications, in which the tandem DNA-based duplication of a gene segment creates an intronic sequence; Intron Transposition, in which a noncoding sequence transposes or gets spliced into an intron-less position in DNA sequence or in a transcript that then reverse transcribed and integrated in the genome; Intron Retention, in which an intron of the parent gene is not spliced out during transcript processing and gets transposed with the retrogene; and Intronization, in which polymorphism in exonic sequences creates functional splice sites converting exonic sequences into introns. Some of the introns in the targeted retrogenes originated by post-integration transposition process; and thus have no 
sequence homology to parental sequences. However, have high sequence homology to multiple $A$. lyrata-specific intergenic regions. The rest of the introns had high sequence homology to parental introns with existence in the exact order arguing for intron retention rather than intron transfer. Interestingly, retained introns would provide longer region of homology between the extrachromosomal retrogene copy and the parental gene favoring homologous recombination. Intron-less genes were shown to respond rapidly to abiotic stress (Jeffares et al., 2008), but their transcripts have relatively short half-life (Narsai et al., 2007). Retrogenes tend to acquire introns, which significantly increase their mRNA half-life (Figure 16).

Hence, we developed targeted retrogene annotation tool (TRAT); and to our knowledge, we report the first natural in planta gene targeting events. Retrogenes acquisition of introns increases their mRNA half-life. 


\section{Materials and methods}

\subsection{Defining the minimum length of poly(A)-tail in Arabidopsis genome.}

We define the poly $(\mathrm{A})$-tail to be the minimum length of non-random consecutive adenine stretches down-stream of protein coding genes. We calculate the length of consecutive adenine stretches in the 150 and 250 bp downstream of stop codon for genes with and without 3' untranslated regions (UTR), respectively (Figure 18). About $99 \%$ of TAIR10 genes had adenine stretches with a length $<15 \mathrm{nt}$ in their downstream regions, allowing a single non-adenine nucleotide per stretch. Therefore, we considered a $\geq 15$ nt long adenine stretch as a poly(A)-tail. Consequently, genes with such poly $(A)$-tail in their downstream regions were accepted as retrogene candidates.

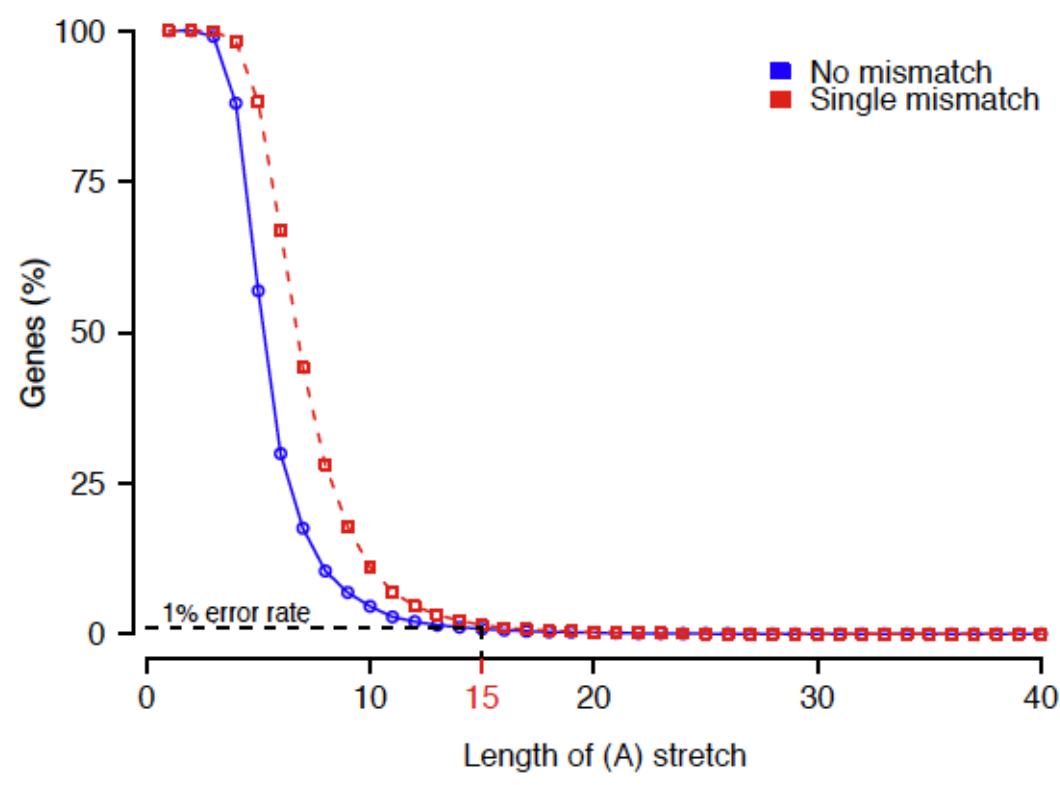

Figure 18. Defining the minimum length of non-random poly(A)-tail in Arabidopsis genome.

The length of consecutive adenine stretches (x-axis) in 150 or 250 bp downstream regions of the stop codons for genes with or without 3'-UTR, respectively (y-axis). Multiple adeninestretches per gene were calculated. The $1 \%$ error rate and a single non-adenine mismatch were accepted. 


\subsection{Genome-wide transcription and mRNA half-life analysis}

All microarray analyses were based on the publicly available datasets. Throughout the study, we used the following ATH1 cDNA microarrays (Affymetrix): wild type $A$. thaliana development produced by the AtGenExpress consortium (Schmid et al., 2005), A. thaliana pollen development and sperm cells datasets NASCARRAYS-48 (Honys and Twell, 2003, 2004), ddm1-12 dataset deposited at the Gene Expression Omnibus (GEO) as the GSE18977 (Baubec et al., 2010), kyp dataset GEO GSE22957 (Inagaki et al., 2010), clf, swn and clf/swn dataset GEO GSE20256 and the hda6 (rts1-1) dataset NASCARRAYS-538 (Popova et al., 2013). The raw data were processed and normalized using the Robust Multi-array Averaging (RMA) method (Irizarry et al., 2003) in R software (www.R-project.org) using Bioconductor (www.bioconductor.org) and the affy package (Gautier et al., 2004). The fie transcription values were retrieved from the GEO dataset GSE19851 (Bouyer et al., 2011) as the normalized transcription values. Retrogene and parent probes that corresponded to multiple gene models were excluded from genome-wide analysis. The transcription borderline for transcribed genes (gcRMA $\geq 5$ ) was based on the minimal density of genes between peaks indicating absent or background signals versus high transcription signals (Figure 3 ). The $A$. thaliana mRNA half-life data and rosette- and pollen-specific RNA sequencing data were extracted from previously published datasets (Narsai et al., 2007; Loraine et al., 2013). Randomized sets of genes or gene pairs were generated, plots drawn and statistical tests calculated in R. Significance of density distributions was tested using the MannWhitney-Wilcoxon (MWW) rank sum test with correction and co-transcription correlation by the Pearson product-moment correlation coefficient $(r)$.

\subsection{Chromatin analysis}

Chromatin data of 10-day-old $A$. thaliana seedlings were retrieved from the publicly available genome-wide atlas of chromatin modifications (Roudier et al., 2011). The frequencies for individual groups were compared. Pearson correlations were calculated in Excel (Microsoft) and heat maps were built in R. 


\subsection{Targeted retrogene annotation tool (TRAT)}

Pairwise interspecies gene orthology was established between 27416, 31606 and 26521 annotated protein coding genes of $A$. thaliana, A. lyrata and C. rubella, respectively depending on protein sequence homology using InParanoid Version 4.1 with default parameters (Remm et al., 2001). Among the 20552 established orthology groups between $A$. thaliana and $A$. Iyrata, 19694 gene pair were identified as syntenic orthologs i-AdHoRe v3.0 (Simillion et al., 2008). In total 473 orthologous pairs have a minimum differential intron number of three, and were considered for further analysis. Genomic (gDNA) and complimentary (cDNA) DNA of the candidate gene pairs were aligned using MUSCLE v3.8.31 (Edgar, 2004); and the similarity of their exon-intron structure was visually evaluated. We then manually confirmed the conserved synteny between the orthologous pair. Finally, we compared their exonintron structure to the syntenic ortholog of the out-group C. rubella (Slotte et al., 2013). The protocol was executed with customized bioperl and awk scripts (Stajich et al., 2002).

\subsection{Nucleotide similarity}

The coding sequences (CDS) of all retrogenes and their parents genes were aligned using MUSCLE v3.8.31 (Edgar, 2004). Nucleotide diversity (NuclDiv) between each aligned pair was calculated using R software (www.R-project.org) and library pegas v0.5-1 in R (Paradis, 2010). Nucleotide similarity was calculated as (1 NuclDiv). Data retrieval, alignment and parsing were done using customized bioperl scripts.

\subsection{Small RNA data}

Small RNA deep sequencing reads were retrieved from (Ma et al., 2010) and mapped against $A$. lyrata reference genome using Bowtie 2 v2.1.0 (Langmead and Salzberg, 2012). The data from three biological samples (2 flowers and one rosette) were averaged and plotted as calculated number of mapped reads per kbp of genetic elements for each category of interest. 


\subsection{RNA deep sequencing experiment}

RNA samples from whole rosettes, floral tissues and shoot apical meristem were harvested from $A$. lyrata MN47 plants grown under controlled ambient conditions for 2 weeks, 4 weeks and 2 days post sowing, respectively. RNA-seq libraries were prepared and indexed from isolated mRNA using Illumina TruSeq RNA Sample preparation kit v2. Libraries were then sequenced using Illumina HiSeq 2000 platform generating on average 17.4 million single-end $100 \mathrm{nt}$-long read per sample. Sequencing reads were mapped against the reference genome using Tophat2 (Kim et al., 2013) and Bowtie 2 (Langmead and Salzberg, 2012). RNA sequencing reads from tissues grown under heat and cold stress growth conditions were also used [provided by B. Pietzenuk, unpublished data, and D. Koenig (Seymour et al., 2014)].

\subsection{Overlap between genes and TEs}

The overlap between repeatmasker-identified TEs and genes was performed with the BEDtools suite (Quinlan and Hall, 2010); as well as the overlap with five 1kilobase pins upstream the transcription start sites and downstream transcription termination sites. 


\section{APPENDICES}

\subsection{Appendix A. Comprehensive list of $A$. thaliana retrogenes}

\begin{tabular}{|c|c|c|c|c|c|c|c|c|c|c|}
\hline \multicolumn{2}{|c|}{ Gene ID } & \multicolumn{3}{|c|}{ Introns } & \multirow{2}{*}{$\begin{array}{c}\text { CDS } \\
\text { nucleotide } \\
\text { similarity (\%) }\end{array}$} & \multirow{2}{*}{ PolyA-tail } & \multirow{2}{*}{$\begin{array}{c}\text { Retrocopies } \\
\text { per parent }\end{array}$} & \multicolumn{3}{|c|}{ Study } \\
\hline retrogene & parent & retrogene & parent & $\begin{array}{c}\text { differenc } \\
\mathrm{e}\end{array}$ & & & & $\begin{array}{l}\text { Zhang } \\
(2005)\end{array}$ & $\begin{array}{c}\text { Zhu } \\
(2009)\end{array}$ & $\begin{array}{l}\text { This } \\
\text { study }\end{array}$ \\
\hline AT1G01300 & AT5G10770 & 0 & 2 & 2 & 59,8 & 0 & 1 & 0 & 0 & 1 \\
\hline AT1G02000 & AT4G10960 & 0 & 11 & 11 & 59,4 & 0 & 7 & 0 & 0 & 1 \\
\hline AT1G03020 & AT5G63030 & 0 & 3 & 3 & 58,9 & 0 & 2 & 0 & 0 & 1 \\
\hline AT1G03300 & AT2G47230 & 1 & 7 & 6 & 75,8 & 0 & 1 & 1 & 1 & 0 \\
\hline AT1G03390 & AT5G41040 & 0 & 3 & 3 & 57,7 & 0 & 1 & 0 & 0 & 1 \\
\hline AT1G05020 & AT4G02650 & 0 & 2 & 2 & 57,2 & 0 & 1 & 0 & 0 & 1 \\
\hline AT1G06370 & AT5G14850 & pseudogene & 8 & NA & 73,6 & 1 & 1 & 0 & 0 & 1 \\
\hline AT1G08120 & AT5G26110 & 0 & 6 & 6 & 92,9 & 0 & 1 & 0 & 1 & 1 \\
\hline AT1G08250 & АT3G07630 & 0 & 11 & 11 & 62,1 & 0 & 4 & 1 & 0 & 1 \\
\hline AT1G11050 & AT1G70520 & 0 & 6 & 6 & 59,8 & 0 & 1 & 0 & 0 & 1 \\
\hline AT1G11090 & AT2G39420 & 0 & 7 & 7 & 59,3 & 0 & 1 & 0 & 0 & 1 \\
\hline AT1G11980 & AT3G52590 & 0 & 4 & 4 & 61,9 & 0 & 1 & 0 & 0 & 1 \\
\hline AT1G12310 & AT2G27030 & 0 & 2 & 2 & 62,3 & 0 & 1 & 0 & 0 & 1 \\
\hline AT1G14430 & AT5G19580 & 0 & 1 & 1 & 57,3 & 1 & 1 & 0 & 0 & 1 \\
\hline AT1G14680 & AT4G09060 & 0 & 5 & 5 & 79,5 & 0 & 1 & 1 & 1 & 1 \\
\hline AT1G15000 & AT5G22980 & 0 & 8 & 8 & 57,8 & 0 & 1 & 0 & 0 & 1 \\
\hline AT1G15040 & AT1G66860 & 1 & 4 & 3 & 69,5 & 0 & 1 & 1 & 1 & 0 \\
\hline AT1G15700 & AT2G33040 & 0 & 7 & 7 & 58,6 & 0 & 1 & 0 & 0 & 1 \\
\hline AT1G15720 & AT5G58340 & 0 & 5 & 5 & 78,8 & 0 & 1 & 1 & 1 & 1 \\
\hline AT1G16390 & AT1G73220 & 0 & 1 & 1 & 59,7 & 1 & 1 & 0 & 0 & 1 \\
\hline AT1G16550 & AT3G01610 & pseudogene & 8 & NA & 90,1 & 0 & 2 & 0 & 0 & 1 \\
\hline AT1G18480 & AT1G07010 & 0 & 9 & 9 & 60,1 & 0 & 1 & 0 & 0 & 1 \\
\hline AT1G18970 & AT1G09560 & 0 & 1 & 1 & 60,4 & 1 & 1 & 0 & 0 & 1 \\
\hline AT1G19810 & AT3G01610 & pseudogene & 8 & NA & 68,1 & 0 & 2 & 1 & 0 & 1 \\
\hline AT1G20000 & AT4G20280 & 1 & 4 & 3 & 80,3 & 0 & 1 & 0 & 1 & 0 \\
\hline AT1G24530 & AT2G26060 & 0 & 9 & 9 & 57 & 0 & 1 & 0 & 0 & 1 \\
\hline AT1G25390 & AT1G66880 & 3 & 8 & 5 & 65,1 & 0 & 1 & 0 & 0 & 1 \\
\hline AT1G26220 & AT1G32070 & 0 & 7 & 7 & 59,4 & 0 & 1 & 0 & 0 & 1 \\
\hline AT1G27190 & AT5G48380 & 0 & 2 & 2 & 58,9 & 0 & 1 & 0 & 0 & 1 \\
\hline AT1G28760 & AT5G67610 & 0 & 9 & 9 & 57,7 & 0 & 1 & 0 & 0 & 1 \\
\hline AT1G29260 & AT5G58230 & 0 & 5 & 5 & 58,8 & 0 & 1 & 0 & 0 & 1 \\
\hline AT1G29340 & AT3G46510 & 0 & 3 & 3 & 58,6 & 0 & 1 & 0 & 0 & 1 \\
\hline AT1G29780 & AT5G11860 & 0 & 4 & 4 & 56,9 & 0 & 1 & 0 & 0 & 1 \\
\hline AT1G30360 & AT1G32090 & 5 & 10 & 5 & 58,1 & 0 & 1 & 0 & 0 & 1 \\
\hline AT1G30455 & AT2G45100 & 1 & 14 & 13 & 92,8 & 0 & 1 & 0 & 1 & 0 \\
\hline AT1G31814 & AT5G48385 & 0 & 4 & 4 & 57,4 & 0 & 1 & 0 & 0 & 1 \\
\hline AT1G31900 & AT1G56000 & pseudogene & 9 & NA & 73,6 & 0 & 1 & 0 & 0 & 1 \\
\hline AT1G32090 & AT1G58520 & 10 & 16 & 6 & 58,9 & 0 & 1 & 0 & 0 & 1 \\
\hline AT1G32480 & AT4G35260 & 0 & 3 & 3 & 77,5 & 0 & 1 & 0 & 0 & 1 \\
\hline AT1G33280 & AT4G17980 & 2 & 4 & 2 & 62,5 & 0 & 3 & 0 & 0 & 1 \\
\hline AT1G33612 & AT5G49750 & 0 & 13 & 13 & 57,2 & 0 & 1 & 0 & 0 & 1 \\
\hline AT1G33740 & AT5G56500 & pseudogene & 14 & NA & 80,3 & 0 & 1 & 0 & 0 & 1 \\
\hline
\end{tabular}




\begin{tabular}{|c|c|c|c|c|c|c|c|c|c|c|}
\hline AT1G34130 & AT5G19690 & 4 & 22 & 18 & 63,2 & 0 & 1 & 0 & 1 & 0 \\
\hline AT1G35440 & AT4G19560 & 0 & 6 & 6 & 62,7 & 0 & 1 & 1 & 0 & 1 \\
\hline AT1G43895 & AT1G43890 & pseudogene & 7 & NA & 64,8 & 0 & 1 & 0 & 0 & 1 \\
\hline AT1G45100 & АT3G10845 & 1 & 16 & 15 & 78,4 & 0 & 1 & 1 & 1 & 0 \\
\hline AT1G50060 & AT1G50050 & 1 & 3 & 2 & 80,7 & 0 & 1 & 0 & 0 & 1 \\
\hline AT1G47056 & AT4G15475 & 0 & 7 & 7 & 58,2 & 0 & 2 & 0 & 0 & 1 \\
\hline AT1G53010 & AT4G35840 & 0 & 4 & 4 & 58,1 & 0 & 1 & 0 & 0 & 1 \\
\hline AT1G53170 & AT5G07310 & 0 & 1 & 1 & 58 & 1 & 1 & 0 & 0 & 1 \\
\hline AT1G53345 & AT5G09580 & 0 & 7 & 7 & 58,9 & 0 & 1 & 1 & 0 & 1 \\
\hline AT1G54660 & AT5G23960 & pseudogene & 6 & NA & 70,7 & 0 & 1 & 0 & 0 & 1 \\
\hline AT1G54985 & AT1G74190 & pseudogene & 4 & NA & 67 & 0 & 1 & 0 & 0 & 1 \\
\hline AT1G55035 & AT3G06720 & pseudogene & 10 & NA & 82,2 & 0 & 1 & 1 & 0 & 1 \\
\hline AT1G55390 & AT5G42280 & 0 & 2 & 2 & 82 & 0 & 1 & 0 & 0 & 1 \\
\hline AT1G55928 & AT2G27285 & 0 & 3 & 3 & 79 & 0 & 1 & 0 & 0 & 1 \\
\hline AT1G56070 & AT1G06220 & 3 & 11 & 8 & 58 & 0 & 1 & 0 & 0 & 1 \\
\hline AT1G57740 & AT4G18465 & pseudogene & 22 & NA & 69,5 & 0 & 1 & 0 & 0 & 1 \\
\hline AT1G58330 & AT1G77920 & 0 & 8 & 8 & 57,1 & 0 & 1 & 0 & 0 & 1 \\
\hline AT1G60480 & AT1G10630 & pseudogene & 6 & NA & 76,7 & 0 & 1 & 0 & 0 & 1 \\
\hline AT1G60660 & AT1G26340 & 0 & 2 & 2 & 64,2 & 0 & 1 & 0 & 0 & 1 \\
\hline AT1G61410 & AT3G23100 & 0 & 5 & 5 & 94,1 & 0 & 1 & 1 & 0 & 0 \\
\hline AT1G63210 & AT1G65440 & 1 & 19 & 18 & 81,2 & 0 & 1 & 1 & 0 & 0 \\
\hline AT1G63760 & AT1G05890 & pseudogene & 15 & NA & 94,2 & 0 & 1 & 1 & 0 & 1 \\
\hline AT1G64780 & AT2G38290 & 0 & 4 & 4 & 55,6 & 0 & 1 & 0 & 0 & 1 \\
\hline AT1G65210 & AT4G38030 & 1 & 12 & 11 & 88,1 & 0 & 1 & 1 & 0 & 0 \\
\hline AT1G66770 & AT4G10850 & 0 & 4 & 4 & 81 & 0 & 1 & 1 & 1 & 1 \\
\hline AT1G68610 & AT1G14870 & 0 & 3 & 3 & 59,9 & 0 & 1 & 1 & 1 & 1 \\
\hline AT1G70430 & AT5G14720 & 17 & 20 & 3 & 65,7 & 0 & 2 & 0 & 0 & 1 \\
\hline AT1G70460 & AT5G56890 & 7 & 13 & 6 & 61 & 0 & 1 & 0 & 0 & 1 \\
\hline AT1G71090 & AT5G01990 & 0 & 8 & 8 & 59,6 & 0 & 1 & 0 & 0 & 1 \\
\hline AT1G72820 & AT5G15640 & 0 & 5 & 5 & 60,3 & 0 & 1 & 0 & 0 & 1 \\
\hline AT1G73050 & AT1G72970 & 2 & 6 & 4 & 59 & 0 & 1 & 0 & 1 & 0 \\
\hline AT1G73500 & AT5G56580 & 0 & 7 & 7 & 57,3 & 0 & 2 & 0 & 0 & 1 \\
\hline AT1G74550 & AT2G40890 & 0 & 2 & 2 & 64,1 & 0 & 1 & 0 & 0 & 1 \\
\hline AT1G76090 & AT5G13710 & 0 & 13 & 13 & 59,8 & 0 & 1 & 0 & 0 & 1 \\
\hline AT1G77130 & AT3G18660 & 2 & 4 & 2 & 66,9 & 0 & 1 & 0 & 1 & 0 \\
\hline AT1G77920 & AT1G08320 & 8 & 12 & 4 & 62,8 & 0 & 1 & 0 & 0 & 1 \\
\hline AT1G80510 & AT5G38820 & 0 & 4 & 4 & 61,6 & 0 & 1 & 0 & 0 & 1 \\
\hline AT2G01180 & AT3G02600 & 1 & 8 & 7 & 64,2 & 0 & 1 & 1 & 1 & 0 \\
\hline AT2G01372 & AT3G13062 & pseudogene & 6 & NA & 75,8 & 0 & 1 & 0 & 0 & 1 \\
\hline AT2G03410 & AT5G47540 & 0 & 10 & 10 & 67,9 & 0 & 1 & 1 & 0 & 1 \\
\hline AT2G04120 & AT5G59150 & pseudogene & 2 & NA & 70,2 & 0 & 1 & 0 & 0 & 1 \\
\hline AT2G04280 & AT3G56750 & 0 & 5 & 5 & 58,9 & 0 & 1 & 0 & 0 & 1 \\
\hline AT2G10735 & AT5G45573 & pseudogene & 4 & NA & 65,4 & 0 & 1 & 0 & 0 & 1 \\
\hline AT2G11280 & AT1G63640 & pseudogene & 20 & NA & 64,2 & 0 & 1 & 0 & 0 & 1 \\
\hline AT2G16830 & AT2G16850 & pseudogene & 3 & NA & 65,2 & 0 & 1 & 0 & 0 & 1 \\
\hline AT2G18940 & AT1G74850 & 0 & 3 & 3 & 54,7 & 0 & 1 & 0 & 0 & 1 \\
\hline AT2G19250 & AT1G08520 & pseudogene & 14 & NA & 60,3 & 0 & 1 & 0 & 0 & 1 \\
\hline AT2G19550 & АT3G47590 & 0 & 4 & 4 & 77,7 & 0 & 1 & 1 & 1 & 1 \\
\hline AT2G21060 & AT1G75560 & 0 & 3 & 3 & 56,8 & 1 & 2 & 0 & 0 & 1 \\
\hline AT2G22760 & AT4G37850 & 2 & 4 & 2 & 62,9 & 1 & 1 & 0 & 0 & 1 \\
\hline
\end{tabular}




\begin{tabular}{|c|c|c|c|c|c|c|c|c|c|c|}
\hline AT2G24410 & AT3G20550 & 0 & 10 & 10 & 84,8 & 0 & 1 & 1 & 0 & 1 \\
\hline АT2G24748 & AT4G00260 & pseudogene & 6 & NA & 78,7 & 0 & 1 & 0 & 0 & 1 \\
\hline АT2G25500 & AT5G66550 & 0 & 8 & 8 & 88,9 & 0 & 1 & 1 & 0 & 0 \\
\hline AT2G25630 & AT2G44450 & 1 & 11 & 10 & 86,6 & 0 & 3 & 1 & 0 & 0 \\
\hline АT2G26060 & AT5G67320 & 9 & 13 & 4 & 58,2 & 0 & 1 & 0 & 0 & 1 \\
\hline АT2G26490 & AT5G52820 & 0 & 11 & 11 & 58,6 & 0 & 1 & 0 & 0 & 1 \\
\hline AT2G27820 & АT3G07630 & 0 & 11 & 11 & 63 & 0 & 4 & 1 & 0 & 0 \\
\hline AT2G28420 & AT1G80160 & 0 & 2 & 2 & 64,6 & 0 & 1 & 0 & 0 & 1 \\
\hline АT2G28850 & AT2G32440 & 0 & 8 & 8 & 57,1 & 0 & 2 & 0 & 0 & 1 \\
\hline АT2G29160 & AT2G29350 & pseudogene & 4 & NA & 72 & 0 & 1 & 0 & 0 & 1 \\
\hline AT2G31230 & AT1G43160 & 0 & 1 & 1 & 62,3 & 1 & 1 & 0 & 0 & 1 \\
\hline AT2G32050 & AT1G17130 & 0 & 7 & 7 & 71,8 & 0 & 2 & 1 & 0 & 1 \\
\hline AT2G32510 & AT1G09000 & 0 & 16 & 16 & 60,6 & 0 & 2 & 0 & 0 & 1 \\
\hline AT2G33580 & АT3G21630 & 0 & 11 & 11 & 54,8 & 0 & 1 & 0 & 0 & 1 \\
\hline AT2G34760 & AT2G34770 & pseudogene & 6 & NA & 61,2 & 0 & 1 & 0 & 0 & 1 \\
\hline AT2G34850 & AT4G10960 & 5 & 11 & 6 & 60,7 & 0 & 7 & 0 & 0 & 1 \\
\hline AT2G34960 & AT4G21120 & 0 & 2 & 2 & 62,1 & 0 & 1 & 0 & 0 & 1 \\
\hline AT2G35180 & AT3G10950 & pseudogene & 3 & NA & 78,6 & 0 & 1 & 0 & 0 & 1 \\
\hline AT2G36500 & AT5G50530 & 1 & 14 & 13 & 65,6 & 0 & 2 & 1 & 0 & 0 \\
\hline AT2G37970 & AT3G10130 & 0 & 7 & 7 & 57,6 & 0 & 1 & 0 & 0 & 1 \\
\hline AT2G38310 & AT1G01360 & 0 & 2 & 2 & 60,6 & 0 & 1 & 0 & 0 & 1 \\
\hline АT2G38980 & АT3G03720 & pseudogene & 14 & NA & 62,3 & 0 & 1 & 0 & 0 & 1 \\
\hline AT2G40925 & AT2G40910 & 0 & 2 & 2 & 79,1 & 1 & 1 & 0 & 0 & 1 \\
\hline АT2G42850 & AT2G32440 & 2 & 8 & 6 & 57,2 & 0 & 2 & 0 & 0 & 1 \\
\hline AT2G43030 & AT3G17465 & 0 & 4 & 4 & 60 & 0 & 1 & 0 & 0 & 1 \\
\hline AT2G44630 & AT2G41360 & 0 & 4 & 4 & 62,1 & 0 & 1 & 0 & 0 & 1 \\
\hline AT2G45310 & AT4G10960 & 0 & 11 & 11 & 56,6 & 0 & 7 & 0 & 0 & 1 \\
\hline АТЗG01630 & AT1G31470 & 0 & 2 & 2 & 69,8 & 0 & 1 & 0 & 0 & 1 \\
\hline АТЗG02270 & AT5G19485 & 0 & 12 & 12 & 58,4 & 0 & 1 & 0 & 0 & 1 \\
\hline AT3G03160 & AT3G17780 & 0 & 3 & 3 & 55,9 & 0 & 2 & 1 & 0 & 1 \\
\hline АТЗG04700 & AT2G31560 & 1 & 3 & 2 & 62,3 & 0 & 1 & 0 & 0 & 1 \\
\hline АТЗG04790 & AT5G44520 & 0 & 8 & 8 & 61,5 & 0 & 1 & 0 & 0 & 1 \\
\hline АТЗG07730 & AT1G77270 & 0 & 4 & 4 & 62,8 & 0 & 1 & 0 & 0 & 1 \\
\hline АТЗG09375 & AT3G19760 & pseudogene & 6 & NA & 88,2 & 0 & 1 & 0 & 0 & 1 \\
\hline АT3G10400 & AT5G64200 & 0 & 8 & 8 & 60,9 & 0 & 1 & 0 & 0 & 1 \\
\hline AT3G11810 & AT2G03330 & 0 & 2 & 2 & 91,8 & 1 & 1 & 1 & 0 & 0 \\
\hline AT3G12630 & AT1G51200 & 0 & 2 & 2 & 60,7 & 0 & 1 & 0 & 0 & 1 \\
\hline АТЗG12910 & AT4G17980 & 2 & 4 & 2 & 57,5 & 0 & 3 & 0 & 0 & 1 \\
\hline АТ3G14370 & AT2G26700 & 0 & 2 & 2 & 60 & 1 & 1 & 0 & 0 & 1 \\
\hline AT3G14440 & АT3G63520 & 0 & 13 & 13 & 57,7 & 0 & 1 & 0 & 0 & 1 \\
\hline AT3G16510 & AT4G34150 & 0 & 6 & 6 & 56,9 & 0 & 1 & 0 & 0 & 1 \\
\hline AT3G18190 & AT1G24510 & 0 & 8 & 8 & 55,8 & 0 & 1 & 0 & 0 & 1 \\
\hline AT3G18420 & AT4G39470 & 0 & 3 & 3 & 57,3 & 0 & 1 & 0 & 0 & 1 \\
\hline АT3G21220 & AT5G56580 & 0 & 7 & 7 & 56,7 & 0 & 2 & 0 & 0 & 1 \\
\hline АT3G21933 & AT3G22010 & pseudogene & 1 & NA & 72,5 & 0 & 1 & 0 & 0 & 1 \\
\hline АT3G22060 & АT3G21960 & 1 & 4 & 3 & 64,9 & 0 & 1 & 0 & 0 & 1 \\
\hline \multicolumn{11}{|l|}{ АТ3G23780, } \\
\hline АТЗG $18090^{1}$ & AT4G21710 & 7 & 24 & 17 & $56.9,58.1$ & 0 & 2 & 0 & 0 & 1 \\
\hline АT3G23820 & AT4G10960 & 0 & 11 & 11 & 56,4 & 0 & 7 & 0 & 0 & 1 \\
\hline АT3G24330 & AT2G19440 & 0 & 2 & 2 & 63,6 & 0 & 1 & 0 & 0 & 1 \\
\hline
\end{tabular}




\begin{tabular}{|c|c|c|c|c|c|c|c|c|c|c|}
\hline AT3G24500 & AT3G58680 & 0 & 3 & 3 & 64,5 & 0 & 1 & 1 & 0 & 0 \\
\hline АТ3G24927 & AT2G32670 & pseudogene & 4 & NA & 57 & 0 & 1 & 0 & 0 & 1 \\
\hline АТЗG25210 & AT2G27800 & 0 & 2 & 2 & 59,2 & 0 & 1 & 0 & 0 & 1 \\
\hline АT3G25495 & AT5G24280 & pseudogene & 37 & NA & 60,6 & 0 & 1 & 0 & 0 & 1 \\
\hline АТЗG27710 & AT4G34370 & 0 & 5 & 5 & 78,5 & 0 & 2 & 1 & 1 & 1 \\
\hline АТЗG27720 & AT4G34370 & 3 & 5 & 2 & 73,5 & 0 & 2 & 1 & 0 & 0 \\
\hline AT3G27750 & AT5G09320 & 0 & 6 & 6 & 63,1 & 0 & 1 & 0 & 0 & 1 \\
\hline AT3G28720 & AT5G58100 & 0 & 24 & 24 & 56,9 & 0 & 1 & 0 & 0 & 1 \\
\hline АТЗG29380 & AT3G10330 & 1 & 6 & 5 & 57,3 & 0 & 1 & 0 & 0 & 1 \\
\hline AT3G32316 & AT1G01530 & pseudogene & 1 & NA & 67,7 & 0 & 1 & 0 & 0 & 1 \\
\hline AT3G43250 & AT1G17130 & 0 & 7 & 7 & 70,7 & 0 & 2 & 1 & 0 & 1 \\
\hline AT3G43251 & AT5G26880 & pseudogene & 6 & NA & 77,3 & 0 & 1 & 0 & 0 & 1 \\
\hline AT3G44717 & AT5G03495 & pseudogene & 6 & NA & 76,5 & 0 & 1 & 0 & 0 & 1 \\
\hline АТ3G44720 & AT3G07630 & 0 & 11 & 11 & 60,4 & 0 & 4 & 1 & 0 & 0 \\
\hline AT3G45950 & AT1G65660 & 0 & 9 & 9 & 82,8 & 0 & 1 & 1 & 0 & 1 \\
\hline AT3G46510 & AT2G28830 & 3 & 6 & 3 & 70,2 & 0 & 1 & 0 & 0 & 1 \\
\hline AT3G46730 & AT1G58410 & 0 & 2 & 2 & 57,5 & 0 & 1 & 0 & 0 & 1 \\
\hline AT3G47180 & AT3G63530 & 0 & 8 & 8 & 60,2 & 0 & 1 & 0 & 0 & 1 \\
\hline АТЗG47520 & AT5G09660 & 1 & 7 & 6 & 63,6 & 0 & 1 & 0 & 1 & 0 \\
\hline АТЗG49162 & АT2G23900 & pseudogene & 4 & NA & 74,7 & 0 & 1 & 0 & 0 & 1 \\
\hline АТЗG49480 & AT1G05080 & 0 & 2 & 2 & 64,6 & 0 & 1 & 0 & 0 & 1 \\
\hline АТЗG49750 & AT5G21090 & 0 & 5 & 5 & 59 & 0 & 1 & 0 & 0 & 1 \\
\hline AT3G51110 & AT5G41770 & 0 & 6 & 6 & 82,5 & 0 & 1 & 0 & 0 & 1 \\
\hline AT3G52350 & AT5G08535 & 0 & 4 & 4 & 82,6 & 0 & 1 & 0 & 0 & 1 \\
\hline AT3G52950 & AT5G50530 & 1 & 14 & 13 & 64,5 & 0 & 2 & 1 & 0 & 0 \\
\hline АТ3G52960 & AT1G65980 & 0 & 3 & 3 & 62 & 0 & 1 & 0 & 0 & 1 \\
\hline AT3G53640 & AT1G13350 & 0 & 10 & 10 & 89,7 & 0 & 1 & 1 & 1 & 1 \\
\hline АТ3G54900 & AT3G15660 & 0 & 6 & 6 & 61,2 & 0 & 1 & 0 & 0 & 1 \\
\hline АT3G55430 & AT5G24318 & 1 & 3 & 2 & 56,1 & 0 & 1 & 0 & 0 & 1 \\
\hline АТ3G55950 & AT1G70460 & 0 & 7 & 7 & 58,7 & 0 & 1 & 0 & 0 & 1 \\
\hline АТЗG57210 & АТЗ 25080 & 1 & 4 & 3 & 69,6 & 0 & 1 & 0 & 0 & 1 \\
\hline AT3G58330 & AT3G58380 & 1 & 4 & 3 & 63,9 & 0 & 1 & 0 & 0 & 1 \\
\hline AT3G58390 & AT4G27650 & 0 & 16 & 16 & 86,9 & 0 & 1 & 1 & 0 & 1 \\
\hline АT3G60610 & AT1G60170 & pseudogene & 7 & NA & 95,3 & 0 & 1 & 1 & 0 & 1 \\
\hline AT3G60955 & AT3G60950 & pseudogene & 21 & NA & 59,2 & 0 & 1 & 0 & 0 & 1 \\
\hline АT3G60960 & AT5G28340 & 2 & 5 & 3 & 77 & 0 & 1 & 0 & 0 & 1 \\
\hline АТЗG60980 & АТЗG60960 & 0 & 2 & 2 & 61,4 & 0 & 1 & 0 & 0 & 1 \\
\hline АТ3G62350 & AT1G71320 & 0 & 2 & 2 & 73,3 & 0 & 1 & 0 & 1 & 0 \\
\hline АТ3G63060 & AT5G15440 & 0 & 2 & 2 & 56,5 & 0 & 1 & 0 & 0 & 1 \\
\hline АТ3G63380 & AT4G29900 & 0 & 34 & 34 & 60,4 & 0 & 1 & 1 & 0 & 1 \\
\hline AT4G00110 & AT4G10960 & 0 & 11 & 11 & 57,9 & 0 & 7 & 0 & 0 & 1 \\
\hline AT4G01170 & AT1G21560 & 0 & 4 & 4 & 59,7 & 0 & 1 & 0 & 0 & 1 \\
\hline AT4G02630 & AT1G01540 & 0 & 6 & 6 & 69,2 & 0 & 1 & 1 & 1 & 1 \\
\hline AT4G04693 & AT4G04695 & pseudogene & 7 & NA & 56,9 & 0 & 1 & 0 & 0 & 1 \\
\hline AT4G05053 & AT2G26430 & pseudogene & 6 & NA & 91,3 & 0 & 1 & 0 & 0 & 1 \\
\hline AT4G08136 & AT2G18130 & pseudogene & 6 & NA & 84 & 0 & 1 & 0 & 0 & 1 \\
\hline AT4G09466 & AT4G05430 & 0 & 2 & 2 & 59 & 0 & 1 & 0 & 0 & 1 \\
\hline AT4G12250 & AT4G10960 & 0 & 11 & 11 & 59,7 & 0 & 7 & 0 & 0 & 1 \\
\hline AT4G14250 & AT1G14570 & 3 & 9 & 6 & 65,9 & 0 & 1 & 0 & 1 & 0 \\
\hline AT4G14480 & AT5G14720 & 0 & 20 & 20 & 63,4 & 0 & 2 & 0 & 0 & 1 \\
\hline
\end{tabular}




\begin{tabular}{|c|c|c|c|c|c|c|c|c|c|c|}
\hline AT4G15975 & AT1G33480 & 0 & 2 & 2 & 62,1 & 0 & 1 & 0 & 0 & 1 \\
\hline AT4G16210 & AT1G60550 & 2 & 8 & 6 & 56,6 & 0 & 1 & 0 & 0 & 1 \\
\hline AT4G16580 & AT5G66720 & 0 & 4 & 4 & 66 & 0 & 1 & 1 & 1 & 1 \\
\hline AT4G16680 & AT1G32490 & 3 & 27 & 24 & 72 & 0 & 1 & 0 & 1 & 0 \\
\hline AT4G17160 & AT1G02130 & 4 & 7 & 3 & 59,1 & 0 & 1 & 0 & 0 & 1 \\
\hline AT4G17690 & AT4G37520 & 0 & 3 & 3 & 57,8 & 0 & 2 & 0 & 0 & 1 \\
\hline AT4G17905 & AT4G10150 & 0 & 2 & 2 & 57,5 & 0 & 1 & 0 & 0 & 1 \\
\hline AT4G19540 & АT3G24430 & 7 & 13 & 6 & 64,9 & 0 & 1 & 0 & 0 & 1 \\
\hline AT4G20100 & AT4G36850 & 0 & 10 & 10 & 64,7 & 0 & 1 & 0 & 0 & 1 \\
\hline AT4G20360 & AT4G02930 & 0 & 11 & 11 & 67,8 & 0 & 1 & 0 & 0 & 1 \\
\hline AT4G20860 & AT5G44400 & 0 & 2 & 2 & 60,2 & 0 & 1 & 0 & 0 & 1 \\
\hline AT4G26890 & AT1G09000 & 0 & 16 & 16 & 60,6 & 0 & 2 & 0 & 0 & 1 \\
\hline AT4G29050 & AT1G70110 & 0 & 1 & 1 & 74,1 & 1 & 1 & 0 & 0 & 1 \\
\hline AT4G29120 & AT1G17650 & 0 & 11 & 11 & 55,6 & 0 & 1 & 0 & 0 & 1 \\
\hline AT4G30300 & AT4G19210 & 0 & 12 & 12 & 71,5 & 0 & 1 & 1 & 0 & 0 \\
\hline AT4G30440 & AT4G10960 & 0 & 11 & 11 & 59,8 & 0 & 7 & 0 & 0 & 1 \\
\hline AT4G33460 & AT1G65410 & 4 & 9 & 5 & 60,6 & 0 & 1 & 0 & 0 & 1 \\
\hline AT4G34470 & AT1G75950 & 0 & 1 & 1 & 78,5 & 1 & 1 & 0 & 0 & 1 \\
\hline AT4G35260 & AT3G09810 & 3 & 6 & 3 & 60,6 & 0 & 1 & 0 & 0 & 1 \\
\hline AT4G35490 & AT1G32990 & 0 & 3 & 3 & 59 & 0 & 1 & 0 & 0 & 1 \\
\hline AT4G35680 & AT4G01590 & 2 & 7 & 5 & 94,5 & 0 & 1 & 0 & 1 & 0 \\
\hline AT4G36020 & AT1G75560 & 0 & 3 & 3 & 57,8 & 0 & 2 & 0 & 0 & 1 \\
\hline AT4G39670 & АT2G34690 & 0 & 3 & 3 & 59,3 & 0 & 1 & 0 & 0 & 1 \\
\hline AT5G01290 & АТЗG09100 & 14 & 16 & 2 & 80,5 & 0 & 1 & 0 & 0 & 1 \\
\hline AT5G01715 & AT5G01720 & pseudogene & 7 & NA & 56,9 & 0 & 1 & 0 & 0 & 1 \\
\hline AT5G02460 & AT3G55370 & 1 & 3 & 2 & 63,8 & 0 & 1 & 0 & 0 & 1 \\
\hline AT5G03980 & AT1G28580 & 1 & 4 & 3 & 58,6 & 1 & 1 & 0 & 1 & 0 \\
\hline AT5G04610 & AT2G31740 & 0 & 15 & 15 & 61,1 & 0 & 1 & 0 & 0 & 1 \\
\hline AT5G07225 & AT5G52140 & 4 & 8 & 4 & 61,7 & 0 & 1 & 0 & 0 & 1 \\
\hline AT5G10400 & AT5G10980 & 0 & 2 & 2 & 80 & 0 & 2 & 0 & 0 & 1 \\
\hline AT5G10600 & AT4G37340 & 0 & 2 & 2 & 58,7 & 0 & 1 & 0 & 0 & 1 \\
\hline AT5G10880 & АТ3G62120 & 2 & 11 & 9 & 75,2 & 0 & 1 & 0 & 1 & 0 \\
\hline AT5G10980 & AT4G40040 & 2 & 4 & 2 & 79,8 & 0 & 1 & 0 & 0 & 1 \\
\hline AT5G12030 & AT1G54050 & 0 & 2 & 2 & 60,8 & 0 & 1 & 0 & 0 & 1 \\
\hline AT5G14900 & AT3G62310 & 0 & 6 & 6 & 80,8 & 0 & 1 & 1 & 0 & 0 \\
\hline AT5G15870 & AT1G18310 & 0 & 4 & 4 & 80,2 & 0 & 1 & 1 & 0 & 1 \\
\hline AT5G16080 & AT5G06570 & 0 & 2 & 2 & 59,3 & 1 & 1 & 0 & 0 & 1 \\
\hline AT5G16510 & АT3G02230 & 1 & 3 & 2 & 61,1 & 0 & 1 & 0 & 1 & 0 \\
\hline AT5G16760 & AT4G08170 & 0 & 9 & 9 & 59,4 & 0 & 1 & 0 & 0 & 1 \\
\hline AT5G17190 & АТЗG17780 & 0 & 3 & 3 & 62,1 & 0 & 2 & 0 & 0 & 1 \\
\hline AT5G17630 & AT5G54800 & 0 & 4 & 4 & 59,8 & 0 & 1 & 1 & 1 & 1 \\
\hline AT5G18202 & AT3G03960 & pseudogene & 12 & NA & 81 & 0 & 1 & 0 & 0 & 1 \\
\hline AT5G18560 & AT3G14230 & 0 & 2 & 2 & 57,9 & 0 & 1 & 0 & 0 & 1 \\
\hline AT5G18600 & AT5G63030 & 0 & 3 & 3 & 57,7 & 0 & 2 & 0 & 0 & 1 \\
\hline AT5G22630 & AT3G07630 & 0 & 11 & 11 & 63 & 0 & 4 & 1 & 0 & 0 \\
\hline AT5G22680 & AT5G22720 & 0 & 8 & 8 & 76,9 & 0 & 1 & 0 & 0 & 1 \\
\hline AT5G23070 & AT3G07800 & 0 & 3 & 3 & 66,2 & 0 & 1 & 0 & 0 & 1 \\
\hline AT5G23600 & AT2G45330 & 0 & 7 & 7 & 96,2 & 0 & 1 & 1 & 1 & 1 \\
\hline AT5G24318 & AT4G34480 & 3 & 5 & 2 & 57,9 & 0 & 1 & 0 & 0 & 1 \\
\hline AT5G25350 & AT4G15475 & 1 & 7 & 6 & 55,8 & 0 & 2 & 0 & 0 & 1 \\
\hline
\end{tabular}




\begin{tabular}{|c|c|c|c|c|c|c|c|c|c|c|}
\hline AT5G26900 & AT4G33270 & 0 & 4 & 4 & 83,4 & 0 & 3 & 1 & 0 & 0 \\
\hline AT5G27080 & AT4G33270 & 1 & 4 & 3 & 83,1 & 0 & 3 & 1 & 0 & 0 \\
\hline AT5G27570 & AT4G33270 & 0 & 4 & 4 & 84,6 & 0 & 3 & 1 & 0 & 0 \\
\hline AT5G28210 & AT5G01290 & 0 & 14 & 14 & 76,5 & 0 & 1 & 1 & 0 & 1 \\
\hline AT5G36270 & AT1G75270 & pseudogene & 2 & NA & 75,1 & 0 & 1 & 0 & 0 & 1 \\
\hline AT5G37445 & AT2G32600 & pseudogene & 6 & NA & 83,6 & 0 & 1 & 1 & 0 & 1 \\
\hline AT5G39840 & AT4G14790 & 1 & 15 & 14 & 60,6 & 0 & 1 & 0 & 1 & 0 \\
\hline AT5G40040 & AT2G27710 & 0 & 4 & 4 & 69,7 & 0 & 1 & 0 & 0 & 1 \\
\hline AT5G40140 & AT2G23140 & 0 & 4 & 4 & 59,3 & 0 & 2 & 0 & 0 & 1 \\
\hline AT5G40250 & АT3G48030 & 0 & 2 & 2 & 63,4 & 0 & 1 & 0 & 0 & 1 \\
\hline AT5G40942 & AT3G27060 & pseudogene & 1 & NA & 82,5 & 0 & 1 & 0 & 0 & 1 \\
\hline AT5G42090 & AT2G01070 & 0 & 4 & 4 & 56,7 & 0 & 1 & 0 & 0 & 1 \\
\hline AT5G42130 & AT4G39460 & 0 & 12 & 12 & 57,4 & 0 & 1 & 0 & 0 & 1 \\
\hline AT5G42260 & AT2G44450 & 0 & 11 & 11 & 86,8 & 0 & 3 & 1 & 0 & 0 \\
\hline AT5G42910 & AT4G34000 & 2 & 5 & 3 & 60,8 & 0 & 1 & 0 & 0 & 1 \\
\hline AT5G44170 & AT1G08125 & 0 & 10 & 10 & 57,4 & 0 & 1 & 0 & 0 & 1 \\
\hline AT5G44640 & AT2G44450 & 0 & 11 & 11 & 87,2 & 1 & 3 & 1 & 0 & 1 \\
\hline AT5G46100 & AT4G01400 & 0 & 3 & 3 & 65 & 0 & 1 & 0 & 0 & 1 \\
\hline AT5G47000 & AT4G37520 & 0 & 3 & 3 & 60 & 1 & 2 & 0 & 0 & 1 \\
\hline AT5G47640 & АТ3G53340 & 0 & 6 & 6 & 67,5 & 0 & 1 & 0 & 0 & 1 \\
\hline AT5G49050 & АT2G47710 & 0 & 3 & 3 & 62,2 & 0 & 1 & 0 & 0 & 1 \\
\hline AT5G49200 & AT5G40880 & 0 & 3 & 3 & 89,7 & 0 & 1 & 1 & 1 & 1 \\
\hline AT5G50820 & AT4G17980 & 2 & 4 & 2 & 59,1 & 0 & 3 & 0 & 0 & 1 \\
\hline AT5G50960 & AT4G19540 & 2 & 7 & 5 & 59,3 & 1 & 1 & 0 & 0 & 1 \\
\hline AT5G52090 & AT5G37150 & 0 & 4 & 4 & 97,7 & 0 & 1 & 1 & 1 & 1 \\
\hline AT5G52415 & AT2G15710 & pseudogene & 6 & NA & 68,1 & 0 & 1 & 0 & 0 & 1 \\
\hline AT5G52940 & AT1G05540 & 0 & 2 & 2 & 60,9 & 0 & 2 & 0 & 0 & 1 \\
\hline AT5G54480 & AT1G21740 & 0 & 3 & 3 & 59,6 & 0 & 1 & 0 & 0 & 1 \\
\hline AT5G54550 & AT1G05540 & 0 & 2 & 2 & 61,8 & 0 & 2 & 0 & 0 & 1 \\
\hline AT5G54661 & AT5G54660 & pseudogene & 1 & NA & 68,3 & 0 & 1 & 0 & 0 & 1 \\
\hline AT5G54940 & AT4G27130 & 2 & 4 & 2 & 74,7 & 0 & 1 & 0 & 1 & 0 \\
\hline AT5G54960 & AT4G33070 & 0 & 4 & 4 & 75,6 & 0 & 1 & 1 & 1 & 1 \\
\hline AT5G56450 & AT3G08580 & 0 & 3 & 3 & 58,6 & 0 & 1 & 0 & 0 & 1 \\
\hline AT5G56720 & AT1G04410 & 1 & 6 & 5 & 66,6 & 0 & 1 & 1 & 1 & 0 \\
\hline AT5G58010 & AT4G02590 & 4 & 6 & 2 & 64,2 & 0 & 1 & 0 & 0 & 1 \\
\hline AT5G58230 & AT2G19520 & 5 & 14 & 9 & 58,9 & 0 & 1 & 0 & 0 & 1 \\
\hline AT5G59630 & AT1G61210 & pseudogene & 18 & NA & 58,7 & 0 & 1 & 0 & 0 & 1 \\
\hline AT5G63070 & AT1G04270 & 0 & 3 & 3 & 71,1 & 0 & 1 & 1 & 1 & 1 \\
\hline AT5G63100 & AT5G44600 & 0 & 7 & 7 & 58 & 0 & 1 & 0 & 0 & 1 \\
\hline AT5G63250 & AT5G35740 & 0 & 2 & 2 & 56,5 & 0 & 1 & 0 & 0 & 1 \\
\hline AT5G63370 & AT1G67580 & 0 & 6 & 6 & 65,7 & 0 & 1 & 1 & 1 & 0 \\
\hline AT5G63900 & AT5G58610 & 0 & 8 & 8 & 63,1 & 0 & 1 & 0 & 0 & 1 \\
\hline AT5G65200 & AT2G23140 & 0 & 4 & 4 & 61,7 & 0 & 2 & 0 & 0 & 1 \\
\hline AT5G65360 & AT5G10980 & 0 & 2 & 2 & 80,3 & 0 & 2 & 0 & 0 & 1 \\
\hline ATCG00190 & AT4G21710 & 0 & 24 & 24 & 57,1 & 0 & 2 & 0 & 0 & 1 \\
\hline ATCG00480 & AT5G08670 & 0 & 8 & 8 & 64,7 & 0 & 1 & 0 & 0 & 1 \\
\hline ATCG00810 & AT4G28360 & 0 & 5 & 5 & 57,9 & 0 & 1 & 0 & 0 & 1 \\
\hline ATCG01050 & ATMG00580 & 0 & 3 & 3 & 57,5 & 0 & 1 & 0 & 0 & 1 \\
\hline ATCG01090 & AT1G16700 & 0 & 8 & 8 & 56 & 0 & 1 & 0 & 0 & 1 \\
\hline ATCG01110 & ATMG00510 & 0 & 4 & 4 & 56,7 & 0 & 1 & 0 & 0 & 1 \\
\hline
\end{tabular}




\subsection{Appendix B. Association of genes with epigenetic marks}

Percentages of retrogenes $(R)$, parents $(P)$ and all genes $(G W)$ with histone modifications and gene body DNA methylation.

\begin{tabular}{|c|c|c|c|c|c|c|c|c|c|c|c|c|c|c|c|}
\hline & \multirow{2}{*}{$\begin{array}{c}\text { Total } \\
n\end{array}$} & \multicolumn{2}{|c|}{ H3K4me2 } & \multicolumn{2}{|c|}{ H2Bub } & \multicolumn{2}{|c|}{ H3K4me3 } & \multicolumn{2}{|c|}{ H3K36me3 } & \multicolumn{2}{|c|}{ H3K27me1 } & \multicolumn{2}{|c|}{ H3K27me3 } & \multicolumn{2}{|c|}{$5 \mathrm{mC}$} \\
\hline & & $\mathrm{n}$ & $\%$ & $\mathrm{n}$ & $\%$ & $\mathrm{n}$ & $\%$ & $\mathrm{n}$ & $\%$ & $\mathrm{n}$ & $\%$ & $n$ & $\%$ & $n$ & $\%$ \\
\hline retrogenes $(R)$ & 183 & 160 & 87,4 & 83 & 45,4 & 133 & 72,7 & 124 & 67,8 & 15 & 8,2 & 60 & 32,8 & 34 & 18,6 \\
\hline genome (GW) & 22616 & 19018 & 84,1 & 10596 & 46,9 & 15126 & 66,9 & 13720 & 60,7 & 3087 & 13,6 & 6909 & 30,5 & 7071 & 31,3 \\
\hline parents $(\mathrm{P})$ & 225 & 214 & 95,1 & 151 & 67,1 & 186 & 82,7 & 176 & 78,2 & 28 & 12,4 & 67 & 29,8 & 85 & 37,8 \\
\hline$R \_l e a f-s p e c i f i c$ & 53 & 52 & 98,1 & 32 & 60,4 & 51 & 96,2 & 50 & 94,3 & 1 & 0,0 & 15 & 28,3 & 12 & 22,6 \\
\hline R_all & 183 & 160 & 87,4 & 83 & 45,4 & 133 & 72,7 & 124 & 67,8 & 15 & 8,2 & 60 & 32,8 & 34 & 18,6 \\
\hline R_pollen-specific & 51 & 44 & 86,3 & 18 & 35,3 & 32 & 62,7 & 28 & 54,9 & 7 & 13,7 & 16 & 31,4 & 9 & 17,6 \\
\hline GW_leaf-specific & 5978 & 5799 & 97,0 & 4167 & 69,7 & 5583 & 93,4 & 5292 & 88,5 & 307 & 5,1 & 1063 & 17,8 & 2014 & 33,7 \\
\hline GW_all & 22616 & 19018 & 84,1 & 10596 & 46,9 & 15126 & 66,9 & 13720 & 60,7 & 3087 & 13,6 & 6909 & 30,5 & 7071 & 31,3 \\
\hline GW_pollen-specific & 5156 & 4089 & 79,3 & 1847 & 35,8 & 2681 & 52,0 & 2333 & 45,2 & 994 & 19,3 & 2022 & 39,2 & 1435 & 27,8 \\
\hline P_leaf-specific & 81 & 80 & 98,8 & 69 & 85,2 & 78 & 96,3 & 77 & 95,1 & 4 & 4,9 & 14 & 17,3 & 30 & 37,0 \\
\hline P_all & 225 & 214 & 95,1 & 151 & 67,1 & 186 & 82,7 & 176 & 78,2 & 28 & 12,4 & 67 & 29,8 & 85 & 37,8 \\
\hline P_pollen-specific & 48 & 46 & 95,8 & 27 & 56,3 & 35 & 72,9 & 30 & 62,5 & 13 & 27,1 & 15 & 31,3 & 18 & 37,5 \\
\hline
\end{tabular}




\subsection{Appendix C. Robust Multiarray Averaging (gcRMA) values}

Transcription quantiles (Q1 to Q4; Q1 - lowly transcribed genes and Q4 - highly transcribed genes) and the group average (M) for genome wide expressed genes, DNA-duplicated genes, retrogenes, parental genes and transposable elements across 49 A. thaliana developmental stages.

\begin{tabular}{|c|c|c|c|c|c|c|c|c|c|c|c|c|c|c|c|c|c|c|c|c|c|c|c|c|c|c|}
\hline \multirow[t]{2}{*}{ ATGE ID } & \multirow[t]{2}{*}{$\begin{array}{l}\text { Developmental } \\
\text { stage/tissue }\end{array}$} & \multicolumn{5}{|c|}{$\begin{array}{l}\text { Genome-wide expressed } \\
\text { genes }\end{array}$} & \multicolumn{5}{|c|}{ DNA duplicated genes } & \multicolumn{5}{|c|}{ retrogens } & \multicolumn{5}{|c|}{ parents } & \multicolumn{5}{|c|}{ transposable elements (TEs) } \\
\hline & & $M$ & Q1 & Q2 & Q3 & Q4 & $M$ & Q1 & Q2 & Q3 & Q4 & $\mathrm{M}$ & Q1 & Q2 & Q3 & Q4 & M & Q1 & Q2 & Q3 & Q4 & $M$ & Q1 & Q2 & Q3 & Q4 \\
\hline ATGE_1 & cotyledon_d7 & 6,6 & 3,5 & 5,6 & 7,4 & 9,7 & 7,6 & 4,3 & 7,2 & 8,6 & 10,5 & 6,6 & 3,4 & 5,3 & 7,8 & 9,9 & 7,4 & 4,2 & 6,8 & 8,1 & 10,2 & 4,5 & 3,3 & 3,8 & 4,4 & 6,4 \\
\hline ATGE_2 & hypocotyl_d7 & 6,6 & 3,6 & 5,8 & 7,5 & 9,6 & 7,8 & 4,6 & 7,4 & 8,7 & 10,7 & 6,4 & 3,1 & 5,1 & 7,5 & 10,1 & 7,5 & 4,6 & 6,9 & 8,1 & 10,3 & 4,2 & 2,9 & 3,5 & 4 & 6,3 \\
\hline ATGE_3 & root_d7 & 6,6 & 4,1 & 5,8 & 7,3 & 9,2 & 7,9 & 5,3 & 7,4 & 8,7 & 10,4 & 6,5 & 3,5 & 5,2 & 7,4 & 9,9 & 7,7 & 4,9 & 7 & 8,4 & 10,3 & 4,2 & 2,9 & 3,5 & 4,1 & 6,2 \\
\hline ATGE_4 & SAM+leaves_d7 & 6,6 & 3,4 & 5,7 & 7,5 & 9,7 & 7,6 & 4,1 & 7,1 & 8,6 & 10,8 & 6,5 & 3,1 & 5,1 & 7,4 & 10,2 & 7,5 & 4,5 & 6,6 & 8,3 & 10,5 & 4,3 & 3 & 3,6 & 4,2 & 6,3 \\
\hline ATGE_5 & leaves1+2_d7 & 6,5 & 3,5 & 5,5 & 7,4 & 9,7 & 7,6 & 4,1 & 7,1 & 8,5 & 10,6 & 6,5 & 3,4 & 5,2 & 7,6 & 10,1 & 7,4 & 4,3 & 6,7 & 8,1 & 10,3 & 4,6 & 3,3 & 4 & 4,5 & 6,4 \\
\hline ATGE_6 & veg. SAM_d7 & 6,6 & 3,5 & 5,8 & 7,5 & 9,6 & 7,6 & 4,2 & 7,1 & 8,6 & 10,8 & 6,4 & 3,2 & 5,1 & 7,2 & 10,3 & 7,5 & 4,5 & 6,6 & 8,3 & 10,5 & 4,3 & 3 & 3,6 & 4,2 & 6,3 \\
\hline ATGE_7 & seedling_d7 & 6,6 & 3,4 & 5,7 & 7,5 & 9,6 & 7,5 & 4,1 & 7 & 8,4 & 10,5 & 6,5 & 3,3 & 5,2 & 7,5 & 9,9 & 7,5 & 4,4 & 6,8 & 8,3 & 10,2 & 4,4 & 3,1 & 3,7 & 4,3 & 6,3 \\
\hline ATGE_8 & SAM transition_d14 & 6,6 & 3,4 & 5,8 & 7,5 & 9,5 & 7,5 & 4 & 7 & 8,5 & 10,6 & 6,3 & 3 & 5,1 & 7,2 & 10,1 & 7,6 & 4,6 & 6,7 & 8,4 & 10,5 & 4,2 & 3 & 3,5 & 4,1 & 6,3 \\
\hline ATGE_9 & roots_d17 & 6,6 & 4 & 5,8 & 7,3 & 9,2 & 7,9 & 5,2 & 7,4 & 8,7 & 10,4 & 6,5 & 3,5 & 5,2 & 7,6 & 9,8 & 7,7 & 4,8 & 7 & 8,4 & 10,3 & 4,1 & 2,9 & 3,5 & 4 & 6,2 \\
\hline ATGE_10 & rosette leaf 4_d10 & 6,5 & 3,5 & 5,6 & 7,5 & 9,7 & 7,5 & 4,1 & 7 & 8,4 & 10,6 & 6,5 & 3,3 & 5,2 & 7,5 & 10 & 7,5 & 4,3 & 6,8 & 8,2 & 10,3 & 4,4 & 3,2 & 3,8 & 4,4 & 6,4 \\
\hline ATGE_12 & rosette leaf 2_d17 & 6,6 & 3,4 & 5,6 & 7,5 & 9,7 & 7,7 & 4,4 & 7,3 & 8,7 & 10,5 & 6,5 & 3,3 & 5,1 & 7,9 & 9,8 & 7,5 & 4,1 & 7 & 8,3 & 10,3 & 4,4 & 3,1 & 3,7 & 4,3 & 6,5 \\
\hline ATGE_13 & rosette leaf 4_d17 & 6,6 & 3,5 & 5,6 & 7,5 & 9,7 & 7,7 & 4,3 & 7,3 & 8,7 & 10,5 & 6,5 & 3,3 & 5,1 & 7,8 & 9,9 & 7,5 & 4,1 & 6,9 & 8,2 & 10,4 & 4,5 & 3,2 & 3,8 & 4,4 & 6,5 \\
\hline ATGE_14 & rosette leaf 6_d17 & 6,6 & 3,5 & 5,6 & 7,5 & 9,7 & 7,7 & 4,3 & 7,3 & 8,7 & 10,5 & 6,6 & 3,4 & 5,2 & 7,8 & 10 & 7,5 & 4,2 & 6,9 & 8,2 & 10,4 & 4,6 & 3,3 & 3,9 & 4,5 & 6,5 \\
\hline ATGE_15 & rosette leaf 8_d17 & 6,6 & 3,4 & 5,6 & 7,5 & 9,7 & 7,7 & 4,2 & 7,3 & 8,7 & 10,6 & 6,5 & 3,3 & 5,1 & 7,7 & 10,1 & 7,5 & 4,3 & 6,9 & 8,2 & 10,4 & 4,4 & 3,2 & 3,8 & 4,4 & 6,4 \\
\hline ATGE_16 & rosette leaf $10 \_d 17$ & 6,6 & 3,5 & 5,6 & 7,5 & 9,7 & 7,7 & 4,2 & 7,2 & 8,6 & 10,7 & 6,5 & 3,3 & 5,1 & 7,6 & 10,1 & 7,5 & 4,3 & 6,8 & 8,1 & 10,4 & 4,5 & 3,3 & 3,9 & 4,5 & 6,4 \\
\hline ATGE_17 & rosette leaf $12 \_d 17$ & 6,6 & 3,4 & 5,6 & 7,5 & 9,7 & 7,6 & 4,1 & 7,1 & 8,6 & 10,7 & 6,5 & 3,2 & 5,1 & 7,5 & 10,1 & 7,5 & 4,3 & 6,8 & 8,2 & 10,4 & 4,4 & 3,2 & 3,8 & 4,4 & 6,4 \\
\hline ATGE_19 & leaf 7_petiole_d17 & 6,6 & 3,4 & 5,6 & 7,5 & 9,7 & 7,7 & 4,2 & 7,3 & 8,7 & 10,7 & 6,5 & 3,3 & 5,1 & 7,6 & 10,1 & 7,5 & 4,4 & 6,7 & 8,2 & 10,3 & 4,4 & 3,2 & 3,8 & 4,4 & 6,4 \\
\hline ATGE_20 & leaf 7_proximal 1/2_d17 & 6,6 & 3,5 & 5,6 & 7,5 & 9,7 & 7,6 & 4,2 & 7,2 & 8,6 & 10,6 & 6,6 & 3,4 & 5,1 & 7,7 & 10 & 7,5 & 4,3 & 6,8 & 8,1 & 10,3 & 4,6 & 3,4 & 4 & 4,7 & 6,5 \\
\hline ATGE_21 & leaf 7_distal 1/2_d17 & 6,6 & 3,5 & 5,6 & 7,5 & 9,7 & 7,7 & 4,2 & 7,3 & 8,6 & 10,5 & 6,6 & 3,4 & 5,1 & 7,8 & 10 & 7,5 & 4,2 & 6,9 & 8,2 & 10,3 & 4,6 & 3,3 & 4 & 4,6 & 6,5 \\
\hline ATGE_22 & rosette_d21 & 6,6 & 3,4 & 5,6 & 7,5 & 9,7 & 7,7 & 4,3 & 7,3 & 8,6 & 10,7 & 6,5 & 3,2 & 5,1 & 7,7 & 10 & 7,5 & 4,3 & 6,8 & 8,2 & 10,4 & 4,4 & 3,2 & 3,8 & 4,4 & 6,4 \\
\hline ATGE_23 & rosette_d22 & 6,6 & 3,4 & 5,6 & 7,5 & 9,8 & 7,7 & 4,2 & 7,2 & 8,6 & 10,7 & 6,4 & 3,3 & 5 & 7,5 & 10 & 7,5 & 4,3 & 6,8 & 8,3 & 10,4 & 4,4 & 3,2 & 3,8 & 4,4 & 6,4 \\
\hline ATGE_24 & rosette_d23 & 6,6 & 3,4 & 5,6 & 7,6 & 9,7 & 7,7 & 4,2 & 7,2 & 8,6 & 10,7 & 6,5 & 3,3 & 5 & 7,6 & 10 & 7,5 & 4,3 & 6,9 & 8,3 & 10,4 & 4,5 & 3,2 & 3,8 & 4,4 & 6,4 \\
\hline ATGE_25 & senescing leaf_d35 & 6,5 & 3,5 & 5,7 & 7,5 & 9,4 & 7,6 & 4,6 & 7,2 & 8,4 & 10,1 & 6,5 & 3,5 & 5,2 & 7,9 & 9,4 & 7,7 & 4,4 & 7,4 & 8,7 & 10,2 & 4,5 & 3,2 & 3,7 & 4,3 & 6,6 \\
\hline ATGE_26 & cauline leaf_d21 & 6,6 & 3,4 & 5,6 & 7,5 & 9,6 & 7,6 & 4,3 & 7,3 & 8,6 & 10,4 & 6,5 & 3,3 & 5,1 & 7,9 & 9,7 & 7,6 & 4,3 & 7,2 & 8,5 & 10,3 & 4,5 & 3,2 & 3,8 & 4,4 & 6,5 \\
\hline ATGE_27 & stem_2nd internode_d21 & 6,6 & 3,5 & 5,7 & 7,5 & 9,6 & 7,8 & 4,5 & 7,5 & 8,8 & 10,5 & 6,4 & 3,3 & 4,8 & 7,7 & 9,7 & 7,6 & 4,7 & 6,8 & 8,3 & 10,3 & 4,4 & 3,1 & 3,8 & 4,4 & 6,4 \\
\hline ATGE_28 & stem_1st internode_d21 & 6,6 & 3,4 & 5,7 & 7,5 & 9,7 & 7,9 & 4,6 & 7,5 & 8,8 & 10,7 & 6,4 & 3,1 & 4,8 & 7,6 & 10 & 7,5 & 4,7 & 6,9 & 8,1 & 10,3 & 4,1 & 2,9 & 3,4 & 3,9 & 6,2 \\
\hline ATGE_29 & SAM inflorescence_d21 & 6,6 & 3,5 & 5,8 & 7,5 & 9,4 & 7,5 & 4 & 7 & 8,5 & 10,6 & 6,3 & 3,1 & 5 & 7,2 & 10 & 7,6 & 4,6 & 6,7 & 8,3 & 10,4 & 4,3 & 3,1 & 3,6 & 4,2 & 6,4 \\
\hline ATGE_31 & pedicels_stage15_d21 & 6,6 & 3,5 & 5,6 & 7,5 & 9,7 & 7,7 & 4,2 & 7,2 & 8,7 & 10,7 & 6,4 & 3,3 & 4,9 & 7,4 & 10 & 7,5 & 4,4 & 6,7 & 8,2 & 10,4 & 4,4 & 3,2 & 3,8 & 4,4 & 6,3 \\
\hline ATGE_32 & flower_stage9_d21 & 6,6 & 3,5 & 5,8 & 7,5 & 9,6 & 7,6 & 4,2 & 7,1 & 8,5 & 10,6 & 6,4 & 3,3 & 5 & 7,3 & 10 & 7,7 & 5 & 6,9 & 8,4 & 10,4 & 4,1 & 2,9 & 3,3 & 3,9 & 6,1 \\
\hline
\end{tabular}




\begin{tabular}{|c|c|c|c|c|c|c|c|c|c|c|c|c|c|c|c|c|c|c|c|c|c|c|c|c|c|c|}
\hline ATGE_33 & flower_stage10_d21 & 6,6 & 3,5 & 5,8 & 7,5 & 9,6 & 7,7 & 4,3 & 7,1 & 8,6 & 10,6 & 6,3 & 3,1 & 5,1 & 7,4 & 9,9 & 7,6 & 4,7 & 7 & 8,3 & 10,3 & 4 & 2,8 & 3,3 & 3,9 & 6,1 \\
\hline ATGE_34 & flower_stage12_d21 & 6,6 & 3,6 & 5,7 & 7,5 & 9,6 & 7,9 & 5,1 & 7,2 & 8,6 & 10,7 & 6,4 & 3,3 & 5,2 & 7,3 & 10 & 7,8 & 5,1 & 7,1 & 8,2 & 10,4 & 4 & 2,8 & 3,3 & 3,9 & 6 \\
\hline ATGE_35 & flower_stage15_d21 & 6,6 & 3,6 & 5,8 & 7,5 & 9,6 & 8 & 5,2 & 7,4 & 8,7 & 10,6 & 6,5 & 3,6 & 5,2 & 7,5 & 9,8 & 7,8 & 5,1 & 7,4 & 8,3 & 10,3 & 4,1 & 2,9 & 3,4 & 3,9 & 6,1 \\
\hline ATGE_36 & sepals_stage12_d21 & 6,6 & 3,4 & 5,7 & 7,5 & 9,7 & 7,7 & 4,5 & 7,3 & 8,6 & 10,6 & 6,5 & 3,3 & 5,2 & 7,7 & 9,8 & 7,6 & 4,4 & 7,2 & 8,3 & 10,3 & 4,2 & 3 & 3,6 & 4,2 & 6,2 \\
\hline ATGE_37 & sepals_stage 15 _d21 & 6,6 & 3,7 & 5,7 & 7,4 & 9,4 & 7,7 & 5 & 7,2 & 8,4 & 10,1 & 6,6 & 3,8 & 5,4 & 7,8 & 9,3 & 7,7 & 4,6 & 7,5 & 8,3 & 10,2 & 4,4 & 3,2 & 3,8 & 4,4 & 6,3 \\
\hline ATGE_39 & petals_stage12_d21 & 6,6 & 3,5 & 5,7 & 7,5 & 9,6 & 7,8 & 4,6 & 7,2 & 8,7 & 10,6 & 6,5 & 3,3 & 5,1 & 7,6 & 9,9 & 7,5 & 4,4 & 6,7 & 8,4 & 10,4 & 4,4 & 3,1 & 3,7 & 4,3 & 6,3 \\
\hline ATGE_40 & petals_stage15_d21 & 6,6 & 3,6 & 5,7 & 7,5 & 9,5 & 7,8 & 4,9 & 7,4 & 8,7 & 10,2 & 6,5 & 3,6 & 5,2 & 7,7 & 9,6 & 7,6 & 4,6 & 7,3 & 8,3 & 10,1 & 4,4 & 3,2 & 3,8 & 4,4 & 6,3 \\
\hline ATGE_41 & stamens_stage12_d21 & 6,6 & 4,3 & 5,7 & 7,1 & 9,1 & 8 & 6,3 & 7,3 & 8,5 & 10,2 & 6,7 & 4,2 & 5,7 & 7,3 & 9,4 & 7,8 & 5,3 & 7,4 & 8,1 & 9,9 & 4,5 & 3,4 & 4 & 4,5 & 6,2 \\
\hline ATGE_42 & stamens_stage15_d21 & 6,6 & 4,1 & 5,7 & 7,2 & 9,2 & 8 & 6,1 & 7,4 & 8,5 & 9,9 & 6,7 & 4,3 & 5,7 & 7,4 & 9,4 & 7,6 & 4,9 & 7,2 & 8,2 & 9,8 & 4,6 & 3,5 & 4 & 4,6 & 6,3 \\
\hline ATGE_43 & pollen & 6,4 & 5,5 & 6 & 6,5 & 7,6 & 7,3 & 6,9 & 6,6 & 7,3 & 8,3 & 6,8 & 6 & 6,6 & 6,6 & 7,9 & 7,2 & 5,8 & 7 & 7,5 & 8,4 & 5,9 & 5,3 & 5,7 & 6 & 6,7 \\
\hline ATGE_45 & carpels_stage12_d21 & 6,6 & 3,4 & 5,8 & 7,5 & 9,6 & 7,7 & 4,2 & 7,3 & 8,6 & 10,7 & 6,4 & 3 & 5 & 7,4 & 10 & 7,7 & 4,9 & 6,7 & 8,5 & 10,4 & 4,2 & 3 & 3,5 & 4,1 & 6,2 \\
\hline ATGE_73 & carpels_stage15_d21 & 6,6 & 3,5 & 5,7 & 7,5 & 9,6 & 7,8 & 4,6 & 7,2 & 8,6 & 10,8 & 6,4 & 3,2 & 5,1 & 7,4 & 10 & 7,8 & 5,2 & 7 & 8,4 & 10,5 & 4,1 & 2,9 & 3,4 & 4,1 & 6 \\
\hline ATGE_76 & silique_stage3 & 6,6 & 3,7 & 5,7 & 7,4 & 9,6 & 7,8 & 5,1 & 7,2 & 8,5 & 10,6 & 6,5 & 3,4 & 5,3 & 7,3 & 9,9 & 7,6 & 4,9 & 7 & 8,1 & 10,3 & 4,2 & 3 & 3,5 & 4,1 & 6,2 \\
\hline ATGE_77 & silique_stage 4 & 6,6 & 3,7 & 5,8 & 7,4 & 9,6 & 7,9 & 5 & 7,3 & 8,6 & 10,6 & 6,5 & 3,1 & 5,3 & 7,5 & 10,1 & 7,7 & 5 & 7,2 & 8 & 10,3 & 4,1 & 2,8 & 3,4 & 4 & 6,1 \\
\hline ATGE_78 & silique_stage 5 & 6,6 & 3,7 & 5,7 & 7,4 & 9,6 & 7,9 & 5,1 & 7,3 & 8,6 & 10,6 & 6,5 & 3,2 & 5,1 & 7,6 & 10,1 & 7,7 & 5 & 7,1 & 8 & 10,4 & 4,1 & 2,9 & 3,4 & 4,1 & 6,1 \\
\hline ATGE_79 & seed_stage6 & 6,6 & 3,9 & 5,8 & 7,3 & 9,3 & 7,7 & 4,9 & 7,1 & 8,4 & 10,4 & 6,4 & 3,4 & 4,8 & 7,4 & 10 & 7,6 & 5,2 & 6,7 & 7,9 & 10,2 & 4,4 & 3,2 & 3,7 & 4,4 & 6,2 \\
\hline ATGE_81 & seed_stage7 & 6,6 & 4,1 & 5,7 & 7,3 & 9,3 & 7,7 & 5,1 & 7,1 & 8,4 & 10,3 & 6,4 & 3,5 & 4,9 & 7,3 & 9,9 & 7,6 & 5,2 & 6,8 & 8 & 10,1 & 4,5 & 3,3 & 3,9 & 4,5 & 6,3 \\
\hline ATGE_82 & seed_stage8 & 6,6 & 4,4 & 5,8 & 7,1 & 9 & 7,5 & 5,2 & 6,9 & 8,1 & 9,8 & 6,6 & 3,9 & 5,2 & 7,5 & 9,6 & 7,6 & 5,2 & 7,1 & 8,1 & 9,7 & 5 & 3,8 & 4,4 & 5,1 & 6,6 \\
\hline ATGE_83 & seed_stage9 & 6,5 & 4,5 & 5,7 & 7 & 8,8 & 7,4 & 5,2 & 6,8 & 7,9 & 9,7 & 6,6 & 4,1 & 5,3 & 7,4 & 9,6 & 7,5 & 5,3 & 7,1 & 8 & 9,6 & 5,3 & 4,2 & 4,8 & 5,4 & 6,7 \\
\hline ATGE_84 & seed_stage 10 & 6,5 & 4,5 & 5,8 & 7 & 8,8 & 7,4 & 5,2 & 6,8 & 7,9 & 9,5 & 6,6 & 4,1 & 5,3 & 7,5 & 9,6 & 7,6 & 5,2 & 7,2 & 8,1 & 9,5 & 5,2 & 4,1 & 4,8 & 5,4 & 6,7 \\
\hline
\end{tabular}




\subsection{Appendix D: RAT-generated list of $A$. lyrata retrogenes}

A detailed list of all parent and retrogene identified by RAT using our enhanced version of $A$. lyrata genome.

\begin{tabular}{|c|c|c|c|c|c|c|c|c|c|c|c|c|}
\hline \multirow{2}{*}{$\begin{array}{l}\text { Retrogene } \\
\text { ID }\end{array}$} & \multirow{2}{*}{$\begin{array}{c}\text { Parent } \\
\text { ID } \\
\end{array}$} & \multicolumn{3}{|c|}{ Introns } & \multirow[b]{2}{*}{$\begin{array}{l}\text { Poly }(\mathrm{A}) \mathrm{Ta} \\
\text { il }\end{array}$} & \multirow[b]{2}{*}{ NuclSimil } & \multirow[b]{2}{*}{$\begin{array}{l}\text { Retrocopies } \\
\text { per parent }\end{array}$} & \multirow[b]{2}{*}{ After split } & \multicolumn{2}{|c|}{$\begin{array}{c}\text { Has } A . \text { thaliana } \\
\text { ortholog }\end{array}$} & \multicolumn{2}{|c|}{$\begin{array}{c}\text { Has C. rubella } \\
\text { ortholog }\end{array}$} \\
\hline & & $\begin{array}{l}\text { Retro } \\
\text { gene }\end{array}$ & Parent & $\begin{array}{l}\mathrm{P}- \\
\mathrm{R}\end{array}$ & & & & & $\begin{array}{c}\text { Retrogen } \\
\mathrm{e} \\
\end{array}$ & Parent & $\begin{array}{c}\text { Retrogen } \\
\mathrm{e} \\
\end{array}$ & Parent \\
\hline AL4G22540 & AL4G26720 & 0 & 8 & 8 & 0 & 0.6 & 4 & 0 & 1 & 1 & 1 & 1 \\
\hline AL4G39710 & AL4G26720 & 2 & 8 & 6 & 0 & 0.6 & 4 & 0 & 1 & 1 & 1 & 1 \\
\hline AL7G43910 & AL4G26720 & 3 & 8 & 5 & 0 & 0.6 & 4 & 0 & 1 & 1 & 1 & 1 \\
\hline AL7G34050 & AL4G26720 & 6 & 8 & 2 & 0 & 0.6 & 4 & 0 & 1 & 1 & 1 & 1 \\
\hline AL6G39240 & AL7G18010 & 0 & 4 & 4 & 0 & 0.8 & 3 & 1 & 0 & 1 & 0 & 1 \\
\hline AL5G22670 & AL7G18010 & 1 & 4 & 3 & 0 & 0.8 & 3 & 1 & 0 & 1 & 0 & 1 \\
\hline AL4G18580 & AL7G18010 & 1 & 4 & 3 & 0 & 0.8 & 3 & 1 & 0 & 1 & 0 & 1 \\
\hline AL7G22860 & AL1G29580 & 0 & 11 & 11 & 0 & 0.6 & 2 & 0 & 1 & 1 & 1 & 1 \\
\hline AL5G19130 & AL3G21330 & 1 & 6 & 5 & 1 & 0.6 & 2 & 0 & 1 & 1 & 0 & 1 \\
\hline AL4G21160 & AL8G17110 & 0 & 11 & 11 & 0 & 0.6 & 2 & 0 & 1 & 1 & 1 & 1 \\
\hline AL1G44670 & AL1G36880 & 9 & 17 & 8 & 0 & 0.6 & 2 & 0 & 1 & 1 & 1 & 1 \\
\hline AL3G40740 & AL1G29580 & 7 & 11 & 4 & 0 & 0.6 & 2 & 0 & 1 & 1 & 1 & 1 \\
\hline AL3G30910 & AL1G36880 & 0 & 17 & 17 & 0 & 0.6 & 2 & 0 & 0 & 1 & 1 & 1 \\
\hline AL5G19050 & AL3G21330 & 1 & 6 & 5 & 1 & 0.6 & 2 & 0 & 0 & 1 & 1 & 1 \\
\hline AL5G41340 & AL8G17110 & 9 & 11 & 2 & 0 & 0.7 & 2 & 1 & 0 & 1 & 0 & 1 \\
\hline AL6G14140 & AL4G25900 & 0 & 15 & 15 & 0 & 0.6 & 1 & 0 & 1 & 1 & 1 & 1 \\
\hline AL6G39580 & AL6G10840 & 0 & 14 & 14 & 0 & 0.8 & 1 & 0 & 1 & 1 & 1 & 1 \\
\hline AL2G34550 & AL6G23910 & 0 & 13 & 13 & 0 & 0.6 & 1 & 0 & 1 & 1 & 1 & 1 \\
\hline AL8G19170 & AL7G11440 & 0 & 12 & 12 & 0 & 0.6 & 1 & 0 & 1 & 1 & 1 & 1 \\
\hline AL7G32990 & AL4G37380 & 0 & 12 & 12 & 0 & 0.9 & 1 & 0 & 1 & 1 & 1 & 1 \\
\hline AL4G21650 & AL3G18710 & 0 & 11 & 11 & 1 & 0.6 & 1 & 0 & 1 & 1 & 1 & 1 \\
\hline AL7G32790 & AL6G48800 & 0 & 11 & 11 & 0 & 0.7 & 1 & 0 & 1 & 1 & 1 & 1 \\
\hline AL8G14680 & AL4G41660 & 0 & 11 & 11 & 0 & 0.9 & 1 & 0 & 1 & 1 & 1 & 1 \\
\hline AL8G39780 & AL8G33780 & 0 & 9 & 9 & 0 & 0.6 & 1 & 0 & 1 & 1 & 1 & 1 \\
\hline AL1G41580 & AL8G42360 & 0 & 9 & 9 & 0 & 0.6 & 1 & 0 & 1 & 1 & 1 & 1 \\
\hline AL8G15510 & AL1G18350 & 0 & 9 & 9 & 0 & 0.6 & 1 & 0 & 1 & 1 & 1 & 1 \\
\hline AL1G30500 & AL1G16930 & 0 & 8 & 8 & 0 & 0.6 & 1 & 0 & 1 & 1 & 1 & 1 \\
\hline AL5G25560 & AL5G44960 & 0 & 8 & 8 & 0 & 0.6 & 1 & 0 & 1 & 1 & 1 & 1 \\
\hline AL2G29120 & AL6G10040 & 0 & 8 & 8 & 0 & 0.6 & 1 & 0 & 1 & 1 & 1 & 1 \\
\hline AL3G14820 & AL8G14980 & 0 & 8 & 8 & 0 & 0.6 & 1 & 0 & 1 & 1 & 1 & 1 \\
\hline AL3G30780 & AL1G39550 & 0 & 8 & 8 & 0 & 0.6 & 1 & 0 & 1 & 1 & 1 & 1 \\
\hline AL8G38860 & AL8G14740 & 0 & 8 & 8 & 0 & 0.6 & 1 & 0 & 1 & 1 & 1 & 1 \\
\hline AL1G26720 & AL3G21440 & 0 & 8 & 8 & 0 & 0.6 & 1 & 0 & 1 & 1 & 1 & 1 \\
\hline AL6G27270 & AL7G17430 & 0 & 8 & 8 & 0 & 0.6 & 1 & 0 & 1 & 1 & 1 & 1 \\
\hline AL1G38670 & AL1G45000 & 0 & 7 & 7 & 0 & 0.6 & 1 & 0 & 1 & 1 & 1 & 1 \\
\hline AL4G26320 & AL1G28970 & 0 & 7 & 7 & 0 & 0.7 & 1 & 0 & 1 & 1 & 0 & 1 \\
\hline AL1G21840 & AL4G35250 & 0 & 7 & 7 & 0 & 0.6 & 1 & 0 & 1 & 1 & 1 & 1 \\
\hline AL3G21400 & AL8G40130 & 0 & 7 & 7 & 0 & 0.6 & 1 & 0 & 1 & 1 & 1 & 1 \\
\hline AL1G12730 & AL1G38350 & 0 & 7 & 7 & 0 & 0.8 & 1 & 0 & 1 & 1 & 1 & 1 \\
\hline
\end{tabular}




\begin{tabular}{|c|c|c|c|c|c|c|c|c|c|c|c|c|}
\hline AL5G23850 & AL7G13720 & 0 & 6 & 6 & 0 & 0.9 & 1 & 0 & 1 & 1 & 0 & 0 \\
\hline AL2G36150 & AL7G33810 & 2 & 7 & 5 & 0 & 0.6 & 1 & 0 & 1 & 1 & 1 & 1 \\
\hline AL2G30940 & AL6G26030 & 0 & 5 & 5 & 0 & 0.6 & 1 & 0 & 1 & 1 & 1 & 1 \\
\hline AL5G16810 & AL7G16670 & 0 & 5 & 5 & 0 & 0.8 & 1 & 0 & 1 & 1 & 1 & 1 \\
\hline AL5G34890 & AL3G27820 & 0 & 5 & 5 & 0 & 0.6 & 1 & 0 & 1 & 1 & 1 & 1 \\
\hline AL2G23570 & AL8G38550 & 0 & 5 & 5 & 0 & 0.6 & 1 & 0 & 1 & 1 & 1 & 1 \\
\hline AL3G47110 & AL1G42630 & 0 & 5 & 5 & 0 & 0.8 & 1 & 0 & 1 & 1 & 1 & 0 \\
\hline AL1G42570 & AL6G22240 & 0 & 4 & 4 & 0 & 0.6 & 1 & 0 & 1 & 1 & 1 & 1 \\
\hline AL2G39280 & AL7G50180 & 0 & 4 & 4 & 0 & 0.6 & 1 & 0 & 1 & 1 & 1 & 1 \\
\hline AL1G26320 & AL6G40550 & 0 & 4 & 4 & 0 & 0.8 & 1 & 0 & 1 & 1 & 1 & 1 \\
\hline AL3G31090 & AL7G11450 & 0 & 4 & 4 & 0 & 0.6 & 1 & 0 & 1 & 1 & 1 & 1 \\
\hline AL4G39920 & AL3G29980 & 0 & 4 & 4 & 0 & 0.6 & 1 & 0 & 1 & 1 & 1 & 1 \\
\hline AL6G13490 & AL1G41240 & 0 & 4 & 4 & 1 & 0.6 & 1 & 0 & 1 & 1 & 1 & 0 \\
\hline AL6G28200 & AL8G29320 & 0 & 4 & 4 & 0 & 0.6 & 1 & 0 & 1 & 1 & 1 & 1 \\
\hline AL7G37270 & AL8G43400 & 0 & 4 & 4 & 0 & 0.7 & 1 & 0 & 1 & 1 & 1 & 1 \\
\hline AL7G37950 & AL7G36970 & 2 & 6 & 4 & 0 & 0.6 & 1 & 0 & 1 & 1 & 1 & 0 \\
\hline AL1G12840 & AL7G47230 & 0 & 3 & 3 & 0 & 0.6 & 1 & 0 & 1 & 1 & 1 & 1 \\
\hline AL1G27490 & AL8G33340 & 0 & 3 & 3 & 0 & 0.8 & 1 & 0 & 1 & 1 & 1 & 1 \\
\hline AL8G38800 & AL1G13790 & 0 & 3 & 3 & 0 & 0.7 & 1 & 0 & 1 & 1 & 1 & 1 \\
\hline AL1G42150 & AL5G24530 & 0 & 3 & 3 & 0 & 0.6 & 1 & 0 & 1 & 1 & 1 & 1 \\
\hline AL2G26130 & AL1G26570 & 0 & 3 & 3 & 0 & 0.6 & 1 & 0 & 1 & 1 & 1 & 1 \\
\hline AL3G39070 & AL5G39180 & 0 & 3 & 3 & 0 & 0.6 & 1 & 0 & 1 & 1 & 1 & 1 \\
\hline AL6G33630 & AL3G18890 & 0 & 3 & 3 & 0 & 0.7 & 1 & 0 & 1 & 1 & 1 & 1 \\
\hline AL7G11790 & AL4G29490 & 0 & 3 & 3 & 0 & 0.6 & 1 & 0 & 1 & 1 & 1 & 1 \\
\hline AL7G15460 & AL1G46120 & 0 & 3 & 3 & 0 & 0.6 & 1 & 0 & 1 & 1 & 1 & 1 \\
\hline AL8G21750 & AL4G45430 & 0 & 3 & 3 & 0 & 0.6 & 1 & 0 & 1 & 1 & 1 & 1 \\
\hline AL8G28930 & AL1G34340 & 0 & 3 & 3 & 0 & 0.6 & 1 & 0 & 1 & 1 & 1 & 1 \\
\hline AL8G31200 & AL6G23700 & 0 & 3 & 3 & 0 & 0.6 & 1 & 0 & 1 & 1 & 1 & 1 \\
\hline AL6G27720 & AL1G53600 & 0 & 3 & 3 & 0 & 0.6 & 1 & 0 & 1 & 1 & 1 & 1 \\
\hline AL1G12380 & AL8G38750 & 0 & 3 & 3 & 0 & 0.6 & 1 & 0 & 1 & 0 & 1 & 0 \\
\hline AL1G14680 & AL6G49170 & 0 & 2 & 2 & 0 & 0.6 & 1 & 0 & 1 & 1 & 1 & 1 \\
\hline AL2G32880 & AL4G37200 & 0 & 2 & 2 & 0 & 0.6 & 1 & 0 & 1 & 1 & 0 & 1 \\
\hline AL3G10530 & AL1G48290 & 0 & 2 & 2 & 0 & 0.7 & 1 & 0 & 1 & 1 & 1 & 1 \\
\hline AL4G29790 & AL7G32250 & 0 & 2 & 2 & 0 & 0.6 & 1 & 0 & 1 & 1 & 1 & 1 \\
\hline AL5G32720 & AL2G22290 & 0 & 2 & 2 & 0 & 0.6 & 1 & 0 & 1 & 1 & 1 & 1 \\
\hline AL4G34020 & AL8G26980 & 0 & 2 & 2 & 0 & 0.6 & 1 & 0 & 1 & 1 & 1 & 1 \\
\hline AL7G36050 & AL6G24390 & 0 & 2 & 2 & 0 & 0.6 & 1 & 0 & 1 & 1 & 1 & 1 \\
\hline AL7G34120 & AL5G44950 & 0 & 13 & 13 & 1 & 0.6 & 1 & 0 & 1 & 1 & 1 & 1 \\
\hline AL3G47000 & AL3G49220 & 0 & 13 & 13 & 0 & 0.6 & 1 & 0 & 1 & 1 & 1 & 1 \\
\hline AL1G36880 & AL6G33170 & 17 & 27 & 10 & 0 & 0.6 & 1 & 0 & 1 & 1 & 1 & 1 \\
\hline AL3G21240 & AL8G31900 & 0 & 10 & 10 & 0 & 0.6 & 1 & 0 & 1 & 1 & 1 & 1 \\
\hline AL1G29950 & AL3G20310 & 0 & 9 & 9 & 0 & 0.6 & 1 & 0 & 1 & 1 & 1 & 1 \\
\hline AL6G36340 & AL1G53350 & 0 & 9 & 9 & 0 & 0.6 & 1 & 0 & 1 & 1 & 1 & 1 \\
\hline AL4G25820 & AL4G26770 & 0 & 8 & 8 & 0 & 0.5 & 1 & 0 & 1 & 1 & 1 & 1 \\
\hline AL7G22170 & AL6G23900 & 0 & 8 & 8 & 0 & 0.6 & 1 & 0 & 1 & 1 & 1 & 1 \\
\hline AL8G42100 & AL1G18760 & 0 & 8 & 8 & 0 & 0.6 & 1 & 0 & 1 & 1 & 1 & 1 \\
\hline
\end{tabular}




\begin{tabular}{|c|c|c|c|c|c|c|c|c|c|c|c|c|}
\hline AL3G45420 & AL5G25210 & 0 & 8 & 8 & 0 & 0.7 & 1 & 0 & 1 & 0 & 0 & 0 \\
\hline AL6G46890 & AL4G27460 & 0 & 7 & 7 & 0 & 0.6 & 1 & 0 & 1 & 1 & 1 & 1 \\
\hline AL8G42760 & AL7G39130 & 0 & 7 & 7 & 0 & 0.6 & 1 & 0 & 1 & 1 & 1 & 1 \\
\hline AL3G19350 & AL4G33420 & 0 & 7 & 7 & 0 & 0.6 & 1 & 0 & 1 & 1 & 1 & 1 \\
\hline AL1G19350 & AL7G16970 & 0 & 6 & 6 & 0 & 0.6 & 1 & 0 & 1 & 1 & 1 & 1 \\
\hline AL1G40380 & AL3G33920 & 3 & 9 & 6 & 0 & 0.6 & 1 & 0 & 1 & 1 & 1 & 1 \\
\hline AL1G61870 & AL3G22570 & 0 & 6 & 6 & 0 & 0.6 & 1 & 0 & 1 & 1 & 0 & 0 \\
\hline AL6G29420 & AL7G15710 & 0 & 6 & 6 & 0 & 0.6 & 1 & 0 & 1 & 1 & 1 & 1 \\
\hline AL8G15620 & AL7G17130 & 0 & 5 & 5 & 0 & 0.6 & 1 & 0 & 1 & 1 & 1 & 1 \\
\hline AL6G42340 & AL6G23660 & 0 & 5 & 5 & 0 & 0.6 & 1 & 0 & 1 & 1 & 1 & 1 \\
\hline AL5G29410 & AL1G30410 & 0 & 5 & 5 & 0 & 0.6 & 1 & 0 & 1 & 1 & 1 & 1 \\
\hline AL7G40600 & AL4G34690 & 0 & 5 & 5 & 0 & 0.7 & 1 & 0 & 1 & 1 & 1 & 1 \\
\hline AL5G15220 & AL7G15950 & 0 & 5 & 5 & 0 & 0.6 & 1 & 0 & 1 & 0 & 1 & 1 \\
\hline AL6G16340 & AL7G15040 & 0 & 4 & 4 & 0 & 0.6 & 1 & 0 & 1 & 1 & 1 & 1 \\
\hline AL5G44970 & AL2G35660 & 0 & 4 & 4 & 0 & 0.6 & 1 & 0 & 1 & 1 & 1 & 1 \\
\hline AL3G24430 & AL8G31600 & 0 & 4 & 4 & 1 & 0.6 & 1 & 0 & 1 & 1 & 1 & 1 \\
\hline AL6G19840 & AL3G45050 & 0 & 4 & 4 & 0 & 0.6 & 1 & 0 & 1 & 1 & 1 & 1 \\
\hline AL6G33810 & AL3G28350 & 0 & 4 & 4 & 0 & 0.6 & 1 & 0 & 1 & 1 & 0 & 1 \\
\hline AL6G52050 & AL1G11610 & 0 & 4 & 4 & 0 & 0.6 & 1 & 0 & 1 & 1 & 1 & 1 \\
\hline AL3G34150 & AL2G39750 & 0 & 4 & 4 & 0 & 0.6 & 1 & 0 & 1 & 0 & 0 & 0 \\
\hline AL5G14500 & AL1G65470 & 0 & 4 & 4 & 0 & 0.7 & 1 & 0 & 1 & 0 & 1 & 1 \\
\hline AL7G13720 & AL2G21490 & 6 & 9 & 3 & 0 & 0.9 & 1 & 0 & 1 & 1 & 0 & 1 \\
\hline AL1G64270 & AL7G12280 & 0 & 3 & 3 & 0 & 0.8 & 1 & 0 & 1 & 1 & 1 & 1 \\
\hline AL3G48250 & AL6G12540 & 3 & 6 & 3 & 0 & 0.6 & 1 & 0 & 1 & 1 & 1 & 0 \\
\hline AL3G15000 & AL3G16740 & 0 & 3 & 3 & 0 & 0.6 & 1 & 0 & 1 & 1 & 1 & 1 \\
\hline AL3G35610 & AL1G40380 & 0 & 3 & 3 & 0 & 0.6 & 1 & 0 & 1 & 1 & 1 & 1 \\
\hline AL4G33340 & AL8G39940 & 0 & 3 & 3 & 1 & 0.9 & 1 & 0 & 1 & 1 & 0 & 1 \\
\hline AL7G10720 & AL7G41710 & 0 & 3 & 3 & 0 & 0.7 & 1 & 0 & 1 & 1 & 1 & 1 \\
\hline AL5G41390 & AL4G20040 & 0 & 3 & 3 & 0 & 0.7 & 1 & 0 & 1 & 0 & 1 & 1 \\
\hline $\begin{array}{l}\text { AL7G45940 } \\
\text { AL304U100 }\end{array}$ & AL6G14700 & 0 & 3 & 3 & 0 & 0.6 & 1 & 0 & 1 & 0 & 0 & 0 \\
\hline 10 & AL3G15770 & 0 & 3 & 3 & 0 & 0.7 & 1 & 0 & 1 & 0 & 1 & 0 \\
\hline AL7G30850 & AL2G13410 & 9 & 11 & 2 & 0 & 0.7 & 1 & 0 & 1 & 1 & 1 & 1 \\
\hline AL2G38270 & AL2G38230 & 1 & 3 & 2 & 0 & 0.7 & 1 & 0 & 1 & 1 & 1 & 1 \\
\hline AL4G37190 & AL6G14770 & 1 & 3 & 2 & 0 & 0.7 & 1 & 0 & 1 & 1 & 1 & 1 \\
\hline AL1G36430 & AL1G48400 & 0 & 2 & 2 & 0 & 0.7 & 1 & 0 & 1 & 1 & 1 & 1 \\
\hline AL1G62060 & AL6G28130 & 0 & 2 & 2 & 0 & 0.6 & 1 & 0 & 1 & 1 & 1 & 1 \\
\hline AL2G17100 & AL1G20700 & 0 & 2 & 2 & 1 & 0.7 & 1 & 0 & 1 & 1 & 1 & 1 \\
\hline AL3G40210 & AL4G34620 & 0 & 2 & 2 & 0 & 0.6 & 1 & 0 & 1 & 1 & 1 & 1 \\
\hline AL4G23460 & AL2G34100 & 0 & 2 & 2 & 0 & 0.6 & 1 & 0 & 1 & 1 & 1 & 1 \\
\hline AL5G43590 & AL8G29350 & 0 & 2 & 2 & 0 & 0.6 & 1 & 0 & 1 & 1 & 1 & 1 \\
\hline AL6G20800 & AL7G43970 & 0 & 2 & 2 & 0 & 0.6 & 1 & 0 & 1 & 1 & 1 & 1 \\
\hline AL7G13410 & AL6G21320 & 0 & 2 & 2 & 0 & 0.6 & 1 & 0 & 1 & 1 & 1 & 1 \\
\hline AL4G19410 & AL3G13250 & 2 & 4 & 2 & 1 & 0.6 & 1 & 0 & 1 & 1 & 1 & 1 \\
\hline AL5G38810 & AL3G35480 & 1 & 3 & 2 & 0 & 0.7 & 1 & 0 & 1 & 0 & 1 & 0 \\
\hline AL6G26760 & AL1G44770 & 0 & 2 & 2 & 0 & 0.7 & 1 & 0 & 1 & 1 & 1 & 1 \\
\hline AL7G40660 & AL2G28320 & 0 & 16 & 16 & 0 & 0.6 & 1 & 0 & 0 & 0 & 1 & 1 \\
\hline
\end{tabular}




\begin{tabular}{|c|c|c|c|c|c|c|c|c|c|c|c|c|}
\hline AL7G15950 & AL7G22160 & 5 & 14 & 9 & 0 & 0.6 & 1 & 0 & 0 & 0 & 1 & 1 \\
\hline AL5G29500 & AL6G27260 & 0 & 13 & 13 & 0 & 0.6 & 1 & 0 & 0 & 0 & 1 & 1 \\
\hline AL4G27160 & AL6G44850 & 0 & 11 & 11 & 0 & 0.6 & 1 & 0 & 0 & 0 & 1 & 1 \\
\hline AL4G28070 & AL3G35110 & 0 & 11 & 11 & 0 & 0.6 & 1 & 0 & 0 & 0 & 1 & 1 \\
\hline AL5G33540 & AL5G14280 & 0 & 11 & 11 & 0 & 0.8 & 1 & 0 & 0 & 0 & 1 & 1 \\
\hline AL2G28410 & AL5G23750 & 6 & 11 & 5 & 0 & 0.6 & 1 & 0 & 0 & 0 & 1 & 1 \\
\hline AL3G34620 & AL8G31410 & 0 & 7 & 7 & 0 & 0.6 & 1 & 0 & 0 & 0 & 1 & 1 \\
\hline AL6G25230 & AL4G44970 & 0 & 6 & 6 & 0 & 0.8 & 1 & 0 & 0 & 1 & 1 & 0 \\
\hline AL1G21800 & AL2G28410 & 0 & 6 & 6 & 0 & 0.6 & 1 & 0 & 0 & 0 & 1 & 1 \\
\hline AL6G49190 & AL1G10820 & 0 & 6 & 6 & 0 & 0.7 & 1 & 0 & 0 & 0 & 1 & 1 \\
\hline AL8G39120 & AL2G24870 & 0 & 6 & 6 & 0 & 0.7 & 1 & 0 & 0 & 0 & 1 & 1 \\
\hline AL7G17240 & AL3G46010 & 0 & 5 & 5 & 0 & 0.6 & 1 & 0 & 0 & 1 & 1 & 1 \\
\hline AL1G41940 & AL8G33230 & 0 & 5 & 5 & 1 & 0.6 & 1 & 0 & 0 & 0 & 1 & 1 \\
\hline $\begin{array}{l}\text { AL1G47600 } \\
\text { AL22U1007 }\end{array}$ & AL6G34770 & 0 & 4 & 4 & 0 & 0.6 & 1 & 0 & 0 & 0 & 1 & 1 \\
\hline 0 & AL1G30400 & 0 & 4 & 4 & 0 & 0.6 & 1 & 0 & 0 & 0 & 1 & 1 \\
\hline AL4G23820 & AL1G17130 & 1 & 4 & 3 & 0 & 0.7 & 1 & 0 & 0 & 0 & 1 & 1 \\
\hline AL6G22580 & AL8G33950 & 0 & 3 & 3 & 0 & 0.6 & 1 & 0 & 0 & 0 & 1 & 0 \\
\hline AL1G60550 & AL4G19070 & 0 & 2 & 2 & 0 & 0.6 & 1 & 0 & 0 & 0 & 1 & 1 \\
\hline AL4G31820 & AL1G13440 & 0 & 12 & 12 & 0 & 0.9 & 1 & 1 & 0 & 1 & 0 & 1 \\
\hline AL1G63780 & AL3G17590 & 0 & 10 & 10 & 0 & 0.9 & 1 & 1 & 0 & 1 & 0 & 1 \\
\hline $\begin{array}{l}\text { AL1G29240 } \\
\text { AL701U100 }\end{array}$ & $\begin{array}{l}\text { AL6G12550 } \\
\text { AL239U100 }\end{array}$ & 0 & 10 & 10 & 1 & 1 & 1 & 1 & 0 & 1 & 0 & 1 \\
\hline $\begin{array}{c}20 \\
\text { AL126U100 }\end{array}$ & 10 & 0 & 9 & 9 & 0 & 0.7 & 1 & 1 & 0 & 0 & 0 & 0 \\
\hline 30 & AL5G22300 & 3 & 7 & 4 & 0 & 0.7 & 1 & 1 & 0 & 0 & 0 & 1 \\
\hline AL8G23490 & AL2G38760 & 0 & 6 & 6 & 0 & 0.9 & 1 & 1 & 0 & 1 & 0 & 1 \\
\hline AL3G42150 & AL4G37910 & 0 & 6 & 6 & 0 & 0.9 & 1 & 1 & 0 & 0 & 0 & 1 \\
\hline AL5G13900 & AL8G39460 & 0 & 5 & 5 & 0 & 0.8 & 1 & 1 & 0 & 1 & 0 & 0 \\
\hline AL9U11510 & AL1G63720 & 2 & 5 & 3 & 0 & 0.6 & 1 & 1 & 0 & 0 & 0 & 0 \\
\hline AL2G15400 & AL6G39910 & 0 & 4 & 4 & 0 & 0.8 & 1 & 1 & 0 & 1 & 0 & 1 \\
\hline AL4G45600 & AL7G23280 & 0 & 4 & 4 & 0 & 0.6 & 1 & 1 & 0 & 1 & 0 & 1 \\
\hline AL6G22560 & AL5G13210 & 0 & 4 & 4 & 0 & 0.8 & 1 & 1 & 0 & 0 & 0 & 0 \\
\hline AL2G20810 & AL5G41680 & 2 & 4 & 2 & 0 & 0.6 & 1 & 1 & 0 & 1 & 0 & 1 \\
\hline AL6G41920 & AL2G30020 & 0 & 3 & 3 & 0 & 0.6 & 1 & 1 & 0 & 1 & 0 & 1 \\
\hline AL5G31460 & AL6G33790 & 0 & 3 & 3 & 0 & 0.6 & 1 & 1 & 0 & 0 & 0 & 0 \\
\hline AL7G44060 & AL2G33620 & 0 & 2 & 2 & 0 & 0.8 & 1 & 1 & 0 & 1 & 0 & 1 \\
\hline AL1G17240 & AL4G19410 & 0 & 2 & 2 & 0 & 0.7 & 1 & 1 & 0 & 1 & 0 & 1 \\
\hline AL5G16160 & AL5G36700 & 0 & 2 & 2 & 1 & 0.8 & 1 & 1 & 0 & 1 & 0 & 0 \\
\hline AL3G40250 & AL1G43450 & 0 & 2 & 2 & 0 & 0.6 & 1 & 1 & 0 & 0 & 0 & 0 \\
\hline
\end{tabular}

\subsection{Appendix E: Interspecies comparison of NRPD2E2 DNA sequence}

The alignment shows loss of NRPD2E2 introns causing the structure of NRPD2E2 ${ }^{\mathrm{Aly}}{ }^{\mathrm{MN} 47}$ genomic DNA (Aly_NRPD2E2_gDNA) to match that of NRPD2E2 ${ }^{\text {At_Col }}$ cDNA (Ath_NRPD2E2_cDNA). 
Ath_NRPD2E2_gDNA Ath_NRPD2E2_CDNA

Ath NRPD2E2 gDNA Ath_NRPD2E__CDNA Aly_NRPD2E2_gDNA

Ath_NRPD2E2_gDNA Aly_NRPD2E2_gDNA

Ath_NRPD2E2_gDNA Aly_NRPD2E2_gDNA

Ath_NRPD2E2_gDNA Ath_NRPD2E2_CDNA

Ath NRPD2E2 2 DNA Ath_NRPD2E2_CDNA Aly_NRPD2E2_gDNA

Ath_NRPD2E2_gDNA Ath_NRPD2E2_CDNA Aly_NRPD2E2_gDNA

Ath_NRPD2E2_gDNA Ath_NRPD2E2_CDNA Aly_NRPD2E2_gDNA

Ath_NRPD2E2_gDNA Ath_NRPD2E2_CDNA
Aly_NRPD2E2_gDNA

Ath_NRPD2E2_gDNA Ath_NRPD2E2_CDNA Aly_NRPD2E2_gDNA

Ath_NRPD2E2_gDNA Aly_NRPD2E2_gDNA

Ath_NRPD2E2_gDNA Ath_NRPD2E2_CDNA

Ath_NRPD2E2_gDNA Ath_NRPD2E2_CDNA

Ath_NRPD2E2_gDNA Ath_NRPD2E2_CDNA
Aly_NRPD2E2_gDNA

Ath_NRPD2E2_gDNA Ath_NRPD2E2_CDNA Aly_NRPD2E2_gDNA

Ath_NRPD2E2_gDNA Ath_NRPD2E2_CDNA
Aly_NRPD2E2_gDNA

Ath_NRPD2E2_gDNA Ath_NRPD2E2_CDNA

Ath_NRPD2E2_gDNA Ath_NRPD2E2_CDNA Aly_NRPD2E2_gDNA

Ath_NRPD2E2_gDNA Ath_NRPD2E2_CDNA

Ath_NRPD2E2_gDNA Ath_NRPD2E2_CDNA
Aly_NRPD2E2_gDNA Ath_NRPD2E2_gDNA Ath_NRPD2E2_CDNA

Ath_NRPD2E2_gDNA Ath_NRPD2E2_CDNA Aly_NRPD2E2_gDNA

Ath_NRPD2E2_gDNA Ath_NRPD2E2_CDNA
Aly_NRPD2E2_gDNA

Ath_NRPD2E2_gDNA Ath_NRPD2E2_CDNA Aly_NRPD2E2_gDNA

-AATTTCTTCACTTCTCTTTGACTGCTTCG------CTTAACCACTGAAAAAGTGTGCCAAGGGTTTTCTACGTCGAATCT-CTCCGCAT 82

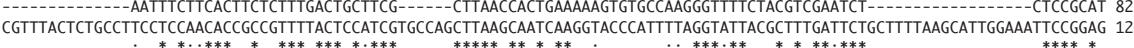
TCTCAG---------CGATTTTCCGGGGACGTTTAC------tCTGCACTCCTCCGACACCG--CCGTTTTACTCCATCGTGCCAGCT--tTAAGCAATCAAGGTACCT 172 TCTCAG---_GATTTCCGGCGACGTTTAC------TCTGCACTCCTCCGACACCG--CCGTTTTACTCCATCGTGCCAGCT--TTAAGCAATCAAG----- 166 ACTATATGCTTTAGAGAATGATTCGGTTCTAGGGGAAGTTTTGATTGCGTGTTTGTATTCGTATGATGCATTTTCGTGGTTCATGATTTTCACGGCTTCTTAATCTTTGTTTG---- 235

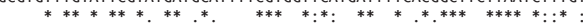

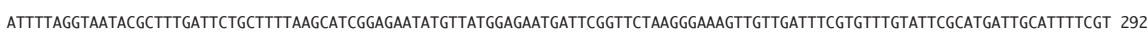

GGTTCATGATTTTCACAGCTTTTTAATCAATTTCTCTGTCTTTGTTTAGGGTTTTTGTTCG--TACAGTGTGTTTTGAGGTATGCCAGATAT---382

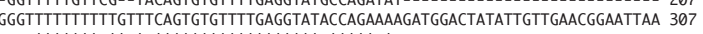
***********:***********************:*:

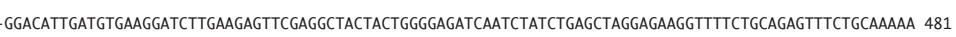
GGACATTGATGTGAAGGATCTTGAGGAGTCGAGGCTACTACTGGGGGATCAATCTATCTGAGCTAGGAGAGGTTTTCTGCAGAGTTTCTGCAAAAA 306 TTTTCTGTTACCAGAAAAGATGGACATTGATGAGATGGATATTGAAGAGATCGAGGCTACTGGG---GAGATCAATCTATCTGAGCTAGGGAGAGTTTTCTCCAGAGTTCTGCAAGA 424

AGCTGCAACTTCTTTCTTTGATAAGTATGGACTTATAAGTCATCAGCTCAACTCCTACAACTACTTCATTGAACACGGGCTTCAGAATGTGTTTCAATCCTTTGGTGAGATGCTTGTGGA 601 AGCTGCAACTTCTTTCTTTGATAAGTATGGACTTATAAGTCATCAGCTCAACTCCTACAACTACTTCATTGAACACGGGCTTCAGAATGTGTTTCAATCCTTTGGTGAGATGCTTGTGGA 426 AGCTGCAACTTCCTTCTTTGATAAGTATGGACTTATAAGTCATCAGCTCAATTCCTACAACTTCTTCATTCAACACGGGCTTCAGGATGTGTTTGAATCCTTTGGTGATATGCTTGTGGA 544

ACCGTCTTTTGATGTTGTAAAGAAGAAGGATAATGATTGGAGATACGCAACGGTGAAGTTCGGAGAAGTCACTGTGGAGAAGCCTACTTTCTTTTCGGATGACAAGGAGCTTGAGTTTCT 721 ACCGTCTT GATGTGTAAAGAAGAAGGATAATGATTGGAGATACGCAACGGTGAAGTTCGGAGAAGTCACTGGGGAGAAGCCTACTTTCTTTTCGGATGACAAGGAGCTTGAGTTTCT 546

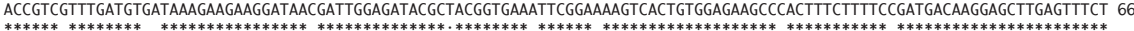

CCCATGGCATGCTAGGCTTCAGAACATGACATACTCTGCAAGGATCAAAGTCAATGTCCAAGTTGAGGTAACAGAAATTCTTTGTCGAAATTAAGTAACCTTGTCTGGATTTGATGAATG 841 CCATGGCATGCTAGGCTTCAGAACATGACATACTCTGCAGGATCAAAGTCAATGTCCAGTTGAG-
CCCATGGCATGCCAGGCTTCAGAACATGACATATTCAGCAAGGATCAAATCAATGTCCAAGTGAG-

ATAAAGAACACATGGTATAAGCTTATTTCTTGATGTTTCTACTAGACTCTTTCTGACACATATATGAAGATGTTGACATACACTGAGGTTCCTGTCATAGATTTCTCAATTTAACTTGCC 961 -

ATCAAATAATTTACTAAGGGTTAAGGAACATATTTGTCTGAAACTGGTTTCACTCTTTTTGGCTTTACAAGTTTTCTGTAATTGGATTTGGTTCCTTATTTGCATTCGCTGGATTTCTTA 1081 -

CCTGAGCAAAATATCTAGTAAAAGAGATTTATTACAGTTACATGTTCGTGTGAAGTAGAGGTGTATTTCAAGCTTGGTTGTGTTTAAGATTGATGATTTTGTCTGCTCCCAATCTTTAGA 1201

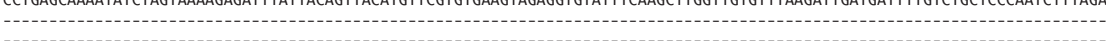

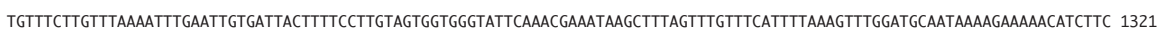

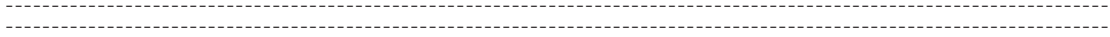

AGCTTTTTTTTTTATTTAGTTCTTCCCCACTGCCTCACTGTGCTTTAGTTTGAGTGTTTTATGCTTGTGTGCAATGACTCTTGTACTGTCAAACTTTTGATGATGTTTCTGTTTTGCTGT 1441 CCATGTATCTTATTCTTATAAATGTAGTTTATTGTCTAACTGCTTCTTCACTCTATAAATTGACTAGGTGTTCAAGAATACTGTTGTTAAAAGCGACAAATTCAAGACAGGACAAGACAA 1561 GTAACAAAATCTTTGTGGAAAATTAAGTAAGCTTGTCTGGATTTGATAA 781

CT-ATGTGGAG---AAGAAGATACTGGATGTCAA -aAagcaggaCattctaAttgGtagcattcctgtcatggtgaAatctatcctttgcaAaAcaagcGagaAag 1662 CT-ATGTCGAG---AAGAAGATACTGGATGTCAA-------AAAGCAGGACATTCTAATTGGTAGCATTCCTGTCATGGTGAAATCTATCCTTTGCAAAACAAGCGAGAAAG 767 ATGATTTCCCTTGCTTGAAACTCAGAAGACCAGTTAACTATCACTTTTTAGTTCAACATATGCAATATGTCTATGTAGTCGAGAGTAAGCTCATTTTTTGATGTTTCTACTAGACTC 901

GGAAAGAAACTGCAAAAAGGG---GGATTGTGCCTTTGATCAGG--GTGGATATTTCGTGATAAAGGGGGTGAGAAGGTGAGTTTAACTAATACATACATATATGCATATTGCCATTC 1777 GGAAGAAACTGCAAAAAGGG--GGATTGTGCCTTTGATCAGG-GTGGATATTTCGTGATAAAGGGGGTGAGAGGTTGCTGACACATATATGAAGATGTTGACATACACTGAGGTTCCTGTCATAGATTTCTCAAACTTATCAAAACCTTTAACT

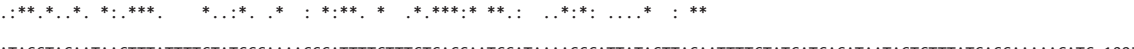
AATACGTACAATAACTTTATTTTCTATGCCAAAACGGATTTTGTTTGTCAGCAATCCATAAAACGGATTATAGTTACAATTTTCTATCATCAGATAATAGTGTTTATCAGCAAAAAGATG 1897 -

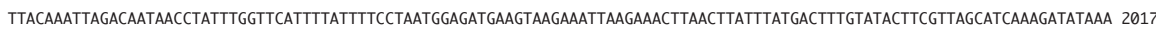
-

CTTTTTCTTTCCTTTCTGGCTTGACCATGAGGCCATGAGTATTCAAATCTTACAGGAAGCGTTCTTTGCAATCTTAGGCTCTGGGACAGATGATTTGACTCTAATATATTCTGGAAAAAA 2137 -

TATTTTCAAGTTGTTATACTTCTCCCTAACGTTATTATATTGTTTTGCAGGTGtTTATAGCTCAAGAACAGATGTGCACAAAGAGACTGTGGATTTCTAATTCACCATGGACAGTCTCCT 2257 -GTGTTTATAGCTCAAGAACAGATGTGCACAAAGAGACTGTGGATTTCTAATTCACCATGGACAGTCTCCT 911 ATAAATAATATATTAAGGGTTATGGCACATATATGTCTGGAAACTGGTTTCACTCTTATTGG-CTTT 1052

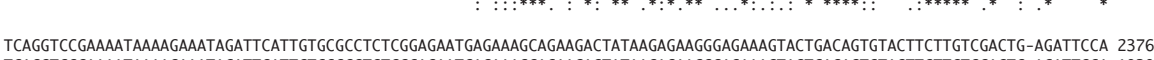
TCAGGTCCGAAAATAAAAGAAATAGATTCATTGTGCGCCTCTCGGAGAATGAGAAAGCAGAAGACTATAAGAGAAGGGAGAAAGTACTGACAGTGTACTTCTTGTCGACTG-AGATTCCA 1030

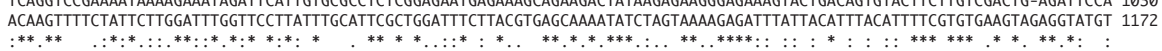

GTCTGGCTCCTCT-TCTTTGCGCTAGGTGTTTCGTCAGACAAAGAAGCCATGGAT-CTAATTGCTTTTGATGGTGATGATGCAAGCATTACCAACAGTCTCATAGCTTCTATCCATGTAG 2494 GTCTGGCTCCTCT-TCTTTGCGCTAGGTGTTTCGTCAGACAAAGAAGCCATGGAT-CTAATTGCTTTTGATGGTGATGATGCAAGCATTACCAACAGTCTCATAGCTTCTATCCATGTAG 1148 TTCAGGCTTCGTTGTTTTTAAGATTGATGATTTTGTCTGCTCCCAATCTTTAGATGTTTCTTGCTTTTTTT----CCGGGCCAAAATTTGAATTGTGATTACTTTTTCTTGTAGTAGTGG 1288 CTGATGCAGTTTGTGAAGCTTTTCGCTGTGGGAACAATGCTTTAACATATGTTG-AACAGCAGATCAAAAGCACCAAATTCCCTCCTGCTGAAAGTGTGGATGAGTGCCTCCATCTGTAT 2613 CTGATGCAGTTTGTGAAGCTTTTCGCTGTGGGAACAATGCTTTAACATATGTTG-AACAGCAGATCAAAAGCACCAAATTCCCTCCTGCTGAAAGTGTGGATGAGTGCCTCCATCTGTAT 1267

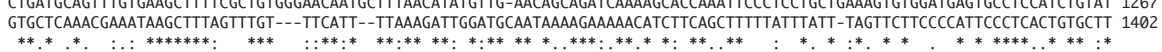
TTGTTTCCAGGCCTCCAAGTTTGAAGAAGAAGCTCGATTCCTGGGCTATATGGTGAGTGCCT-----TCTGAACTCGTATGGGGGAAAAGAAAATGGGAAACAG------GGACA 2722

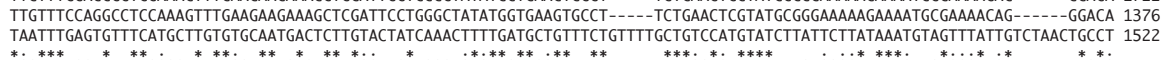


Ath_NRPD2E2_gDNA Aly_NRPD2E2_gDNA

Ath_NRPD2E2_gDNA Ath_NRPD2E2_CDNA

Ath_NRPD2E2_gDNA Ath_NRPD2E2_CDNA

Ath_NRPD2E2_gDNA Ath_NRPD2E2_CDNA Aly_NRPD2E2_gDNA

Ath_NRPD2E2_gDNA Ath_NRPD2E2_CDNA
Aly_NRPD2E2_gDNA

Ath_NRPD2E2_gDNA Ath_NRPD2E2_CDNA
Aly_NRPD2E2_gDNA

Ath_NRPD2E2_gDNA Ath_NRPD2E2_CDNA
Aly_NRPD2E2_gDNA

Ath_NRPD2E2_gDNA Ath_NRPD2E2_CDNA

Ath_NRPD2E2_gDNA Ath_NRPD2E2_CDNA
Aly_NRPD2E2_gDNA

Ath_NRPD2E2_gDNA Ath_NRPD2E2_CDNA

Ath_NRPD2E2_gDNA Ath_NRPD2E2_CDNA
Aly_NRPD2E2_gDNA

Ath_NRPD2E2_gDNA Ath_NRPD2E2_CDNA Aly_NRPD2E2_gDNA

Ath_NRPD2E2_gDNA Aly_NRPD2E2_gDNA

Ath_NRPD2E2_gDNA Ath_NRPD2E2_CDNA
Aly_NRPD2E2_gDNA

Ath_NRPD2E2_gDNA Ath_NRPD2E2_CDNA
Aly_NRPD2E2_gDNA

Ath_NRPD2E2_gDNA Ath_NRPD2E2_CDNA
Aly_NRPD2E2_gDNA

Ath_NRPD2E2_gDNA Ath_NRPD2E2_CDNA
Aly_NRPD2E2_gDNA

Ath_NRPD2E2_gDNA Aty_NRPD2E2_gDNA

Ath_NRPD2E2_gDNA Ath_NRPD2E2_CDNA
Aly_NRPD2E2_gDNA

Ath_NRPD2E2_gDNA Ath_NRPD2E2_CDNA

Ath_NRPD2E2_gDNA Ath_NRPD2E2_CDNA
Aly_NRPD2E2_gDNA

Ath_NRPD2E2_gDNA Ath_NRPD2E2_CDNA

Ath_NRPD2E2_gDNA Ath_NRPD2E2_CDNA
Aly_NRPD2E2_gDNA

GTTTCCGGAATAAGCGAATTGAGCTCGCTGGAGAACTATTGGAGAGGGAGATAAGGGTGCATCTGGCACATGCTAGAAGAAAGATGACCAGGGCCATGCAGAAACACCTCTCAGGCGATG 2842 GTTTCCGGAATAAGCGAATTGAGCTCGCTGGAGAACTATTGGAGAGGGAGATAAGGGTGCATCTGGCACATGCTAGAAGAAAGATGACCAGGGCCATGCAGAAACACCTCTCAGGCGATG 1496

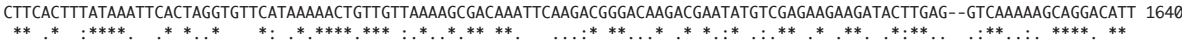

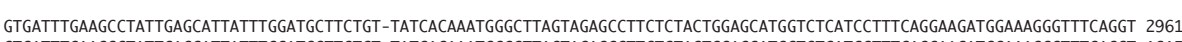
GTGATTTGAAGCCTATTGAGCATTATTTGGATGCTTCTGT-TATCACAAATGGGCTTAGTAGAGCCTTCTCTACTGGAGCATGGTCTCATCCTTTCAGGAAGATGGAAAGGGTTTCAGGT 1615 CTAATTGGTAGCATTCCTGTCATGGTGAAATCTGTCCTTTGCAAAACAAGCGAGAAAGGAAAAGAAAACTG-CAGAAGGGGGATTGTGCCTTTGATCAGGGTGGCTAT--TTTGTGAT 175

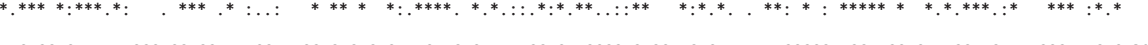
GTTGTGG-CTAATTTGGGTCGTGCAAATCCATTGCAGACTCTGATTGATCTG----AGGAGAACGCGACAGCAAGTCTTATATACCGGCAAGGTTGGAG-ATGCTAGATATCCGTAAGTG 3075 (TGGAG-ATGCTAGATATCCG----- 1723

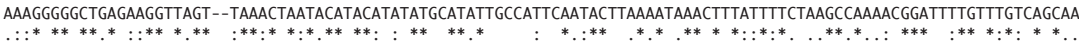

AATTCCACCTCCTCTGGTATATTTAAATATATCTCACGTATTTTAACTTACTGGTCTGGTCTGCATTTACTCCATTTTACATGTCTTCAGACTGTTTTAAAGATATTCGTAATAACTTTA 3195

ATTCAATGTAGTATATGATGCCGATCACTGTTTCTGCAGTCTCTTGTCTGTGTATAATACTTATTTTGTATAGATGTTACTGCTATTAAAAAACTCTGATACTGTCTTTCTTGTTTCTTT 3315

CTCCCTTGGTCAATCTATCTGTTGAACTCTTGAGATTATCCATTTTGGTTCCTTTTCAATGTGAGCGGTTAGACAATTAAATCGTGTTGGGAAACTGAACTATAGCTGCATTGTTTGTAA 3435

TGTTGGCCACAGGCACCCCTCTCACTGGGGCAGAGTATGCTTTTTGTCAACTCCAG-------ACGGTGAAAATTGTGGTCTTGTGAAGAACATGTCTCTTCTGGGACTTGTGAG 3543 -CACCCCTCTCACTGGGGCAGAGTATGCTTTTTGTCAACTCCAG------ACGGTGAAAATTGTGGTCTTGTGAAGAACATGTCTCTTCTGGGACTTGTGAG 1818

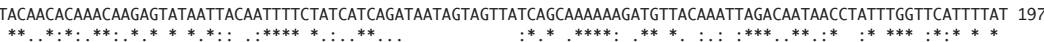

CACCCAAAGTTTGGAGTCTGTGGTGGAAAGCTCTTCGCTTGTGGAATGGAAGAGCTGATGGATGATACATGCACACCATTGTTTGGCAAACATAAAGTTCTTCTCAATGGAGACTGGGT 3663 CACCCAAAGTTTGGAGTCTGTGGTGGAAAAGCTCTTCGCTTGTGGAATGGAAGAGCTGATGGATGATACATGCACACCATTGTTTGGCAAACATAAAGTTCTTCTCAATGGAGACTGGGT 1938 TTTCCTAATGGAGATGGTTAAGAAAGTAAGAAACTTAACTTATTTATGACTTTATATGCTTAAACATACATACAACAAAACCTTT-ATCACACACAAACACATACTGAAGGAAAGGAAAA 2098

GGGATATGTGCAG-ATTCTGAATCCTTTGTCGGGGAGTTAAAAAGCAGGCGGCGCCAAAGTGAATTACCTCGTGAGGTATCTTCTGTTTCAGCAAATCTCTTGCTATATTTTGATATTC 3782

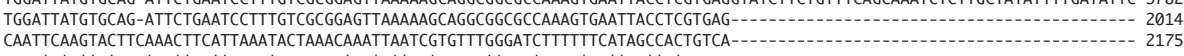

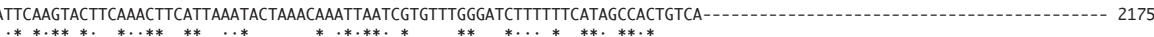

TTGTGTTACTTGGTATTTGCTTTGGATTTTTCTGCTTCAGATGTGTCTATGTCGAATATTGTTTATATATATGAAACGTTCTCTGCAGATGGAAATCAAGCGAGATAAAGATGACAATGA 3902 -ATGGAAATCAAGCGAGATAAAGATGACAATGA 2046 TGGGATTTCTATTTTGAACTATTTTAGTG-GA 2206

GGTAAGAATTTTCACTGATG----CTGGTAGACT-ACTCCGACCTCTCTTG---GTTGTGGAAAATCTCCAAAAGTTGAAGCAAGAAAAACCTTCACAGTATCCT--TTTGACCATCT-- 4010 GGTAAGAATTTTCACTGATG----CTGGTAGACT-ACTCCGACCTCTCTTG---GTTGTGGAAAATCTCCAAAAGTTGAAGCAAGAAAAACCTTCACAGTATCCT--TTTGACCATCT-- 2154 AATTAGTTTTCTCGCGGTTTTCCTTTGCTCAGCAGGCTCGGCTTTGCTTGTCCTTTATGCATACTTTATTAGCATCAAAGATATAGACTTTTTCTTTCCTTTCTGGCTTGACCATGAGG 2326

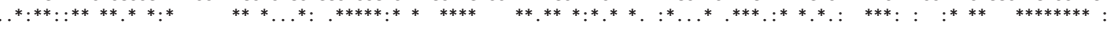

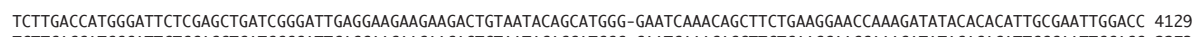
(C)

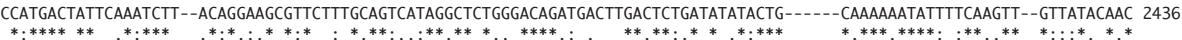

TGTCATTCTTGTTGGGTGTGAGCTGTGCAGTTGTCCCATTTGCAAATCACGACCATGGGAGAAGAGT-TCTCTACCAGTCCCAGAAGCACTGCCAACAAGCCATTGGATTCTCATCAACG 4248 TGTCATTCTTGTTGGGTGTGAGCTGTGCAGTTGTCCCATTTGCAAATCACGACCATGGGAGAAGAGT-TCTCTACCAGTCCCAGAAGCACTGCCAACAAGCCATTGGATTCTCATCAACG 2392

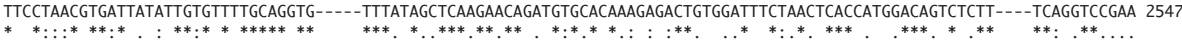
AaCcCTAACATCCGCTGCGATACGCTGTCCCAGCAGCTGTTCTATCCTCAGAAGCCACTGTTCAAGACATTGGCGTCGGAGTGTCtTAAAAAAGAAGTGCTGtTCAATGGCCAGAACGCA 4368 AACCCTAACATCCGCTGCGATACGCTGTCCCAGCAGCTGTTCTATCCTCAGAAGCCACTGTTCAAGACATTGGGGTCGGAGTGTCTTAAAAAAGAAGTGCTGTTCAATGGCCAGAACGCA 2512 AATAAAAGAAATAGGTCATTGTGGG--CCTCTCGGAGATGAGAAATCAGAAGACTAT---AAGAAAGGGAG---AAAGTACTGACAGTGTACTTCTTGTCGACTGAGATTCCAGT 2657

ATTGTTGCTGTGAATGTTCATCTCGGGTACAACCAAGAGGATTCCATTGTGATGAACAAGGCTTCATTGGAACGTGGTATG--TTCCGTTCAGAGCAGATTAGAAGCTACAAAGCAGAGG 4486 ATTGTTGCTGTGAATGTTCATCTGGGGTACAACCAAGAGGATTCCATTGTGATGAACAAGGCTTCATTGGACGGGGTATG--TTCCGTTCAGAGCAGATTAGAAGCTACAAGGCAGAGG 24830 CTGGCTCCTGTTCTT---TGCGCTGGGTGTTTCGTCAG-ACAAAGAAGCCATGAATCTGATTGCTTTGGATGGTGATGATGCAAGCATTACCAACAGTCTCATAGCTTCTATCCA---- 2768

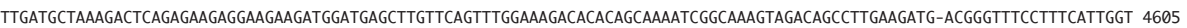
TTGATGCTAAAGACTCAGAGAAGAGGAGGAAGATGGATGAGCTTGTTCAGTTTGGAAAGACACACAGCAAAATCGGCAAAGTAGACAGCCTTGAAGATG-ACGGGTTTCCTTTCATTGGT 2749 -TGAAGCTGATG-CAGTTTGTGAAGCTTTTCGCTGTGGGAACAA-TGCTTTAAGTTATGTTGAACAGCAGATCAAACCTTGGAG---GCCTGGATGACAGGCAAGTATCTCTGACAAGCA 2882

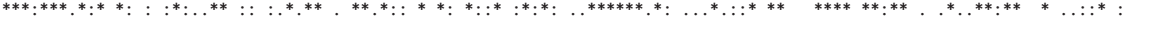
GCTAACATGAGTACTGGGGATATTGTCATTGGCAGATGCACCGAGTCTGGGGCTGATCACAGTATAAAGCTCAAGCACACTGAGAGAGGAATTGTGCAAAAGGGGTATTATCATCTAAT 4725

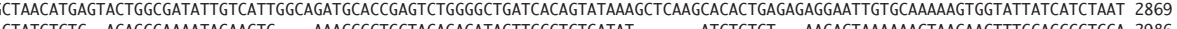

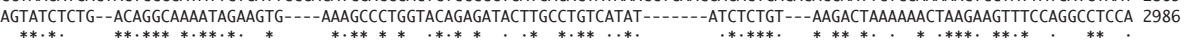
GATGAAGGGAAGAATTTTGCTGCGGTTTCTCTGAGACAGGTAAGTTCCAGATCATACTAAATCGAGCTGTTTTTTCAGAGAATGCATTCCTATGTATGAATCGAATGTTCCATTGATTGG 4845 GATGAAGGGAAGAATTTTGCTGCGGTTTCTCTGAGACAG

AGTACAGAGATATATGACAGGCAAGTATCTCT--GACAG
$. * \ldots * * * *: .: * *:$

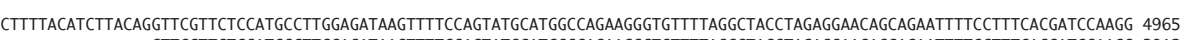
GTTGGTCTCCATGCCTTGGAGATAAGTTTTCCAGTATGCATGGCCAGAAGGGGTTTTAGGCTACCTAGAGGAACAGCAGAATTTTCCTTTCACGATCCAAGG 3012 GCAAAATAGAAG---TGAGCATGAGTT----ATATGACAGGCAAGTAT-CTCTGTAAGACTAAAAAACTAAGAAGTTCAATGTTCTCTGGTTGATTAATAC 311

-CATAGTTCCTGATATTG---TGATAAACCCGCACGCTTTCCCTTCTAGGCAAA-CACCAGGTCAACTCTTGGAGGCTGCTCTCTCCAAAGGAATCGCTTGTCCTATACAAAAGGAGG 5078 --CATAGTTCCTGATATTG---TGATAAACCCGCACGCTTTCCCTTCTAGGCAAA-CACCAGGTCAACTCTTGGAGGCTGCTCTCTCCAAAGGAATCGCTTGTCCTATACAAAAGGAGG 3125

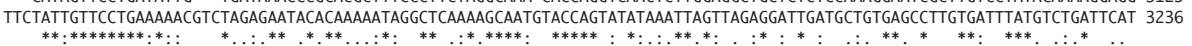

GTAGCTCTGCTGCATACACCAAATTGACACGTCATGCCACTCCTTTCTCCACTCCGGGTGTCACTGAAATCACCGAGCAGCTTCACAGGTACATTCTTCACATTGTCTCTTGGTTTTAGC 5198 GTAGCTCTGCTGCATACACCAAATTGACACGTCATGCCACTCCTTTCTCCACTCCGGGTGTCACTGAAATCACCGAGCAGCTTCACAG--

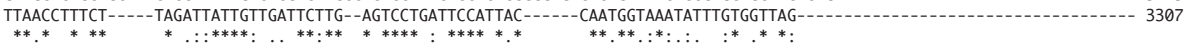

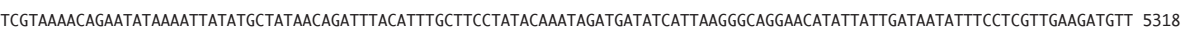
- 
Ath_NRPD2E2_gDNA Ath_NRPD2E2_CDNA Aly_NRPD2E2_gDNA

Ath_NRPD2E2_gDNA Ath_NRPD2E2_CDNA

Ath NRPD2E2 Ath_NRPD2E2_CDNA ALY_NRPD2E2_gDNA

Ath_NRPD2E2_gDNA Ath_NRPD2E2_CDNA Aly_NRPD2E2_gDNA

Ath_NRPD2E2_gDNA Aly_NRPD2E2_gDNA

Ath_NRPD2E2_gDNA Ath_NRPD2E2_CDNA Aly_NRPD2E2_gDNA

Ath_NRPD2E2_gDNA Ath_NRPD2E2_CDNA
Aly_NRPD2E2_gDNA

Ath_NRPD2E2_gDNA Ath_NRPD2E2_CDNA
Aly_NRPD2E2_gDNA

Ath_NRPD2E2_gDNA Ath_NRPD2E2_CDNA
Aly_NRPD2E2_gDNA
TTTTCTAGGAGTACTAGAAGTGAGCATGAGTTATCTGTCTCTGTAAGACTAAAAAACTAAGAAGTTCAATGTTCTATGGTTGATTAATTTCTTGTATTGTGCCTGAAAAACGTCTAGAGA 5558

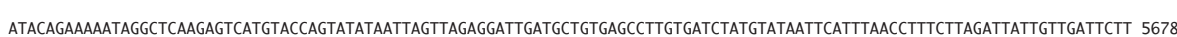

GAGTCCTGATTCATTACCAATGGTAAATATTTGTGATTAGGGCCGGCTTTTCAAGATGGGGAAAGAAAGGGTCTACAACGGTAGATCAGGTGAGATGATGGGTTCTATGATATTCATGG 5798 GGCCGGCTTTTCAAGATGGGGAAACGAAAGGGTCTACAACGGTAGATCAGGTGAGATGATGCGTTCTATGATATTCATGG 3293 GGCCGGCTTTTCAAGATGGGGAAACGAAAGGGTCTACAATGGTAGATCGGGTGAGATGATGCGTTCTCTGATATTCATGG 3387

GCCCAACTTTCTACCAGGGACTTGTCCACATGTCAGAGGACAAAGTCAAGTTCAGGAACACTGGACCAGTCCACCCGCTCACACGCCAGCCAGTTGCAGACAGGAAGAGATTTGGCGGGA 5918 GCCCAACTTTCTACCAGGGACTTGTCCACATGTCAGAGGACAAAGTCAAGTTCAGGAACACTGGACCAGTCCACCCGCTCACAGGCCAGCCAGTTGCAGACAGGAAGAGATTTGGCGGG 341

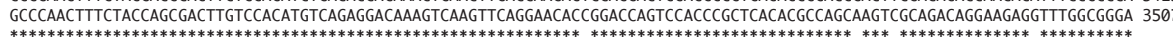

TAAAATTTGGAGAAATGGAGCGAGACTGCCTAATAGCTCACGGTGCATCAGCTAATCTGCATGAGCGTCTCTTCACTCTAAGTGACTCTTCTCAGATGCACATCTGCAGAAAATGTAAGA 603 TAAAATTTGGAGAAATGGAGGGAGACTGCCTAATAGCTCAGGGTGCATCAGCTAATCTGCATGAGCGTCTCTTCACTCTAAGTGACTCTTCTCAGATGCACATCTGCAGAAAATGTAAGA 353 TAAGGTTGGAGAATGGAGCGAGACTGCCTAATAGCTCACGGTGCATCTGCTAATCTGCACGAGCGTCTCTTCACTCTAAGTGACTCTCTCAGATGCACATCTGCAGAAAATGTAAGA 3627
${ }_{*} * * .$.

CCTATGCGAATGtGATCGAGAGGACTCCAAGCAGTGGAAGAAGATTAGAGGGCCATATtGTAGAGTCtGCGTATCCTCAGACCATGTGGTTAGGGTCTATGTTCCGTATGGAGCTAAGC 6158 CCTATGCGAATGTGATCGAGAGGACTCCAAGCAGTGGAAGAAAGATTAGAGGGCCATATTGTAGAGTCTGCGTATCCTCAGACCATGTGGTTAGGGTCTATGTTCCGTATGGAGCTAAGC 365 CCTATGCGAATGTGATCGAGAGGACTCCAAGCAGTGGAAGAAGATCAGAGGGCCATATTGTAGAGTCTGGGTATCCTCAGACCATGTGGTTAGAGTCTATGTTCCGTATGGAGCTAAAC 3747

TTCTGTGTCAGGAGCTGTTCAGCATGGGCATCACTCTCAACTTCGACACCAAGCTATGCTGATTCCCCCTCTTTATTATGTAAATGGCTTATTGCCTTAAGACCATGTTATGTGTAGTTT 6278

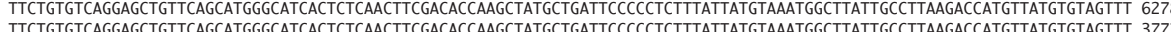
TTCTGTGTCAGGAGGTGTTCAGCATGGGCATCACTCTCAACTTCGACACCAGCTCTGCTGATTACCCCTCTTTATTATGTA*********************************************************,********,*****************10

GCTTCAGTCCCGGTTCTGGTTAGTAGTATAGGTTTTGGTTTGGTTGATTCGGTAAGGGTTATCCGAACCGAAGAAATCGTAAAACCGAGCCACTGATGACTGAACTAACCCGTAAGTGTT 6398 GCTTCAGTCCCGGTTCTGGTTAGTAGTATAGGTTTTGGTTTGGTTGATTCGGTAAGGGTTATCCGAACCGAAGAAATCGTAAAACCGAGCCACTGATGACTGAACTAACCCGTAAGTGTT 3893

GCTTTTGTGAGATTTGACTCTTTAACCGTTAATAATTCTCGGATCTAAAGTAAAGTTTTAGG 6460 GCTTTGTGAGATTTGACTCTTTAACCGTAAATAATTCTGGATCTAAAGTAAAGTTTTAGG 3955 


\section{REFERENCES}

Abdelsamad, A., and Pecinka, A. (2014). Pollen-specific activation of Arabidopsis retrogenes is associated with global transcriptional reprogramming. Plant Cell 26, 3299-3313. sAltschul, S.F., Gish, W., Miller, W., Myers, E.W., and Lipman, D.J. (1990). Basic local alignment search tool. Journal of molecular biology 215, 403-410.

Arabidopsis Genome Initiative (2000). Analysis of the genome sequence of the flowering plant Arabidopsis thaliana. Nature 408, 796-815.

Bai, Y., Casola, C., and Betrán, E. (2008). Evolutionary origin of regulatory regions of retrogenes in Drosophila. BMC Genomics 9, 1-9.

Baubec, T., Dinh, H.Q., Pecinka, A., Rakic, B., Rozhon, W., Wohlrab, B., von Haeseler, A., and Scheid, O.M. (2010). Cooperation of multiple chromatin modifications can generate unanticipated stability of epigenetic states in Arabidopsis. Plant Cell 22, 34-47.

Baumbusch, L.O., Thorstensen, T., Krauss, V., Fischer, A., Naumann, K., Assalkhou, R., Schulz, I., Reuter, G., and Aalen, R.B. (2001). The Arabidopsis thaliana genome contains at least 29 active genes encoding SET domain proteins that can be assigned to four evolutionarily conserved classes. Nucleic Acids Res 29, 4319-4333.

Beck, C.R., Collier, P., Macfarlane, C., Malig, M., Kidd, J.M., Eichler, E.E., Badge, R.M., and Moran, J.V. (2010). LINE-1 retrotransposition activity in human genomes. Cell 141, 1159-1170.

Blanc, G., and Wolfe, K.H. (2004). Functional divergence of duplicated genes formed by polyploidy during Arabidopsis evolution. Plant Cell 16, 1679-1691.

Borg, M., Brownfield, L., Khatab, H., Sidorova, A., Lingaya, M., and Twell, D. (2011). The R2R3 MYB transcription factor DUO1 activates a male germline-specific regulon essential for sperm cell differentiation in Arabidopsis. Plant Cell 23, 534-549.

Borges, F., Calarco, J.P., and Martienssen, R.A. (2012). Reprogramming the epigenome in Arabidopsis pollen. Cold Spring Harbor Symp Quant Biol 77, 1-5.

Boutanaev, A.M., Kalmykova, A.I., Shevelyov, Y.Y., and Nurminsky, D.I. (2002). Large clusters of co-expressed genes in the Drosophila genome. Nature 420,666-669.

Bouyer, D., Roudier, F., Heese, M., Andersen, E.D., Gey, D., Nowack, M.K., Goodrich, J., Renou, J.-P., Grini, P.E., Colot, V., et al. (2011). Polycomb repressive complex 2 controls the embryo-to-seedling phase transition. PLoS Genet 7, e1002014. 
Chartier-Harlin, M.C., Kachergus, J., Roumier, C., Mouroux, V., Douay, X., Lincoln, S., Levecque, C., Larvor, L., Andrieux, J., Hulihan, M., et al. (2004). Alpha-synuclein locus duplication as a cause of familial Parkinson's disease. Lancet 364, 1167-1169.

Chen, X. (2009). Small RNAs and their roles in plant development. Annual review of cell and developmental biology 25, 21-44.

Clauss, M.J., and Koch, M.A. (2006). Poorly known relatives of Arabidopsis thaliana. Trends in plant science 11, 449-459.

Coleman-Derr, D., and Zilberman, D. (2012). Deposition of histone variant H2A.Z within gene bodies regulates responsive genes. PLoS Genet 8, e1002988.

Comai, L. (2005). The advantages and disadvantages of being polyploid. Nat Rev Genet 6 , 836-846.

Cooke, S.L., Shlien, A., Marshall, J., Pipinikas, C.P., Martincorena, I., Tubio, J.M., Li, Y., Menzies, A., Mudie, L., Ramakrishna, M., et al. (2014). Processed pseudogenes acquired somatically during cancer development. Nature communications 5, 3644.

Creasey, K.M., Zhai, J., Borges, F., Van Ex, F., Regulski, M., Meyers, B.C., and Martienssen, R.A. (2014). miRNAs trigger widespread epigenetically activated siRNAs from transposons in Arabidopsis. Nature 508, 411-415.

De Smet, R., Adams, K.L., Vandepoele, K., Van Montagu, M.C.E., Maere, S., and Van de Peer, Y. (2013). Convergent gene loss following gene and genome duplications creates single-copy families in flowering plants. Proc Natl Acad Sci USA 110, 2898-2903.

Dehal, P., and Boore, J.L. (2005). Two rounds of whole genome duplication in the ancestral vertebrate. PLoS Biol 3, e314.

Deng, W., Maust, B.S., Nickle, D.C., Learn, G.H., Liu, Y., Heath, L., Kosakovsky Pond, S.L., and Mullins, J.I. (2010). DIVEIN: a web server to analyze phylogenies, sequence divergence, diversity, and informative sites. BioTechniques 48, 405-408.

Ding, W., Lin, L., Chen, B., and Dai, J. (2006). L1 elements, processed pseudogenes and retrogenes in mammalian genomes. IUBMB life 58, 677-685.

Edgar, R.C. (2004). MUSCLE: multiple sequence alignment with high accuracy and high throughput. Nucleic Acids Res 32, 1792-1797.

Fablet, M., Bueno, M., Potrzebowski, L., and Kaessmann, H. (2009). Evolutionary origin and functions of retrogene introns. Mol Biol Evol 26, 2147-2156.

Farrona, S., Thorpe, F.L., Engelhorn, J., Adrian, J., Dong, X., Sarid-Krebs, L., Goodrich, J., and Turck, F. (2011). Tissue-specific expression of FLOWERING LOCUS T in 
Arabidopsis is maintained independently of polycomb group protein repression. Plant Cell 23, 3204-3214.

Fawcett, J.A., Maere, S., and Van de Peer, Y. (2009). Plants with double genomes might have had a better chance to survive the Cretaceous-Tertiary extinction event. Proc Natl Acad Sci USA 106, 5737-5742.

Fink, G.R. (1987). Pseudogenes in yeast? Cell 49, 5-6.

Flagel, L.E., and Wendel, J.F. (2009). Gene duplication and evolutionary novelty in plants. New Phytol 183, 557-564.

Gautier, L., Cope, L., Bolstad, B.M., and Irizarry, R.A. (2004). affy--analysis of Affymetrix GeneChip data at the probe level. Bioinformatics 20, 307-315.

Gregory, R.T., and Mable, B.K. (2005). Polyploidy in animals. In The evolution of the genome, R.T. Gregory, ed. (Elsevier), pp. 427-483.

Grigoriev, I.V., Nordberg, H., Shabalov, I., Aerts, A., Cantor, M., Goodstein, D., Kuo, A., Minovitsky, S., Nikitin, R., Ohm, R.A., et al. (2012). The genome portal of the Department of Energy Joint Genome Institute. Nucleic Acids Res 40, D26-32.

Hirotsune, S., Yoshida, N., Chen, A., Garrett, L., Sugiyama, F., Takahashi, S., Yagami, K., Wynshaw-Boris, A., and Yoshiki, A. (2003). An expressed pseudogene regulates the messenger-RNA stability of its homologous coding gene. Nature 423, 91-96.

Hoffmann, R.D., and Palmgren, M.G. (2013). Epigenetic repression of male gametophytespecific genes in the Arabidopsis sporophyte. Mol Plant 6, 1176-1186.

Honys, D., and Twell, D. (2003). Comparative analysis of the Arabidopsis pollen transcriptome. Plant Physiol 132, 640-652.

Honys, D., and Twell, D. (2004). Transcriptome analysis of haploid male gametophyte development in Arabidopsis. Genome Biology 5, R85.

Hu, T.T., Pattyn, P., Bakker, E.G., Cao, J., Cheng, J.F., Clark, R.M., Fahlgren, N., Fawcett, J.A., Grimwood, J., Gundlach, H., et al. (2011). The Arabidopsis lyrata genome sequence and the basis of rapid genome size change. Nat Genet 43, 476-481.

Ibarra, C.A., Feng, X., Schoft, V.K., Hsieh, T.-F., Uzawa, R., Rodrigues, J.A., Zemach, A., Chumak, N., Machlicova, A., Nishimura, T., et al. (2012). Active DNA demethylation in plant companion cells reinforces transposon methylation in gametes. Science 337, 13601364.

Innan, H., and Kondrashov, F. (2010). The evolution of gene duplications: classifying and distinguishing between models. Nat Rev Genet 11, 97-108. 
Irimia, M., Rukov, J.L., Penny, D., Vinther, J., Garcia-Fernandez, J., and Roy, S.W. (2008). Origin of introns by 'intronization' of exonic sequences. Trends in genetics : TIG 24, 378-381.

Irizarry, R.A., Hobbs, B., Collin, F., Beazer-Barclay, Y.D., Antonellis, K.J., Scherf, U., and Speed, T.P. (2003). Exploration, normalization, and summaries of high density oligonucleotide array probe level data. Biostatistics 4, 249-264.

Ishizaki, K., Johzuka-Hisatomi, Y., Ishida, S., lida, S., and Kohchi, T. (2013). Homologous recombination-mediated gene targeting in the liverwort Marchantia polymorpha L. Scientific reports 3, 1532.

Jeffares, D.C., Penkett, C.J., and Bahler, J. (2008). Rapidly regulated genes are intron poor. Trends in genetics : TIG 24, 375-378.

Jelesko, J.G., Harper, R., Furuya, M., and Gruissem, W. (1999). Rare germinal unequal crossing-over leading to recombinant gene formation and gene duplication in Arabidopsis thaliana. Proc Natl Acad Sci U S A 96, 10302-10307.

Kaessmann, H. (2010). Origins, evolution, and phenotypic impact of new genes. Genome Res 20, 1313-1326.

Kaessmann, H., Vinckenbosch, N., and Long, M. (2009). RNA-based gene duplication: mechanistic and evolutionary insights. Nat Rev Genet 10, 19-31.

Kanno, T., Huettel, B., Mette, M.F., Aufsatz, W., Jaligot, E., Daxinger, L., Kreil, D.P., Matzke, M., and Matzke, A.J. (2005). Atypical RNA polymerase subunits required for RNAdirected DNA methylation. Nat Genet 37, 761-765.

Kim, D., Pertea, G., Trapnell, C., Pimentel, H., Kelley, R., and Salzberg, S.L. (2013). TopHat2: accurate alignment of transcriptomes in the presence of insertions, deletions and gene fusions. Genome Biol 14, R36.

Konrad, A., Teufel, A.I., Grahnen, J.A., and Liberles, D.A. (2011). Toward a general model for the evolutionary dynamics of gene duplicates. Genome Biol Evol 3, 1197-1209.

Lafos, M., Kroll, P., Hohenstatt, M.L., Thorpe, F.L., Clarenz, O., and Schubert, D. (2011). Dynamic regulation of H3K27 trimethylation during Arabidopsis differentiation. PLoS Genet 7, e1002040.

Langmead, B., and Salzberg, S.L. (2012). Fast gapped-read alignment with Bowtie 2. Nature methods 9, 357-359.

Larkin, M.A., Blackshields, G., Brown, N.P., Chenna, R., McGettigan, P.A., McWilliam, H., Valentin, F., Wallace, I.M., Wilm, A., Lopez, R., et al. (2007). Clustal W and Clustal X version 2.0. Bioinformatics 23, 2947-2948. 
Li, B., Carey, M., and Workman, J.L. (2007). The role of chromatin during transcription. Cell 128, 707-719.

Liu, C., Lu, F., Cui, X., and Cao, X. (2010). Histone methylation in higher plants. Annu Rev Plant Biol 61, 395-420.

Loraine, A.E., McCormick, S., Estrada, A., Patel, K., and Qin, P. (2013). RNA-seq of Arabidopsis pollen uncovers novel transcription and alternative splicing. Plant Physiol 162, 1092-1109.

Lynch, M., and Conery, J.S. (2003). The evolutionary demography of duplicate genes. Journal of structural and functional genomics 3, 35-44.

Ma, Z., Coruh, C., and Axtell, M.J. (2010). Arabidopsis lyrata small RNAs: transient MIRNA and small interfering RNA loci within the Arabidopsis genus. Plant Cell 22, 1090-1103.

Marques, A.C., Dupanloup, I., Vinckenbosch, N., Reymond, A., and Kaessmann, H. (2005). Emergence of young human genes after a burst of retroposition in primates. PLoS Biol 3, e357.

Monk, D., Arnaud, P., Frost, J.M., Wood, A.J., Cowley, M., Martin-Trujillo, A., Guillaumet-Adkins, A., Iglesias Platas, I., Camprubi, C., Bourc'his, D., et al. (2011). Human imprinted retrogenes exhibit non-canonical imprint chromatin signatures and reside in non-imprinted host genes. Nucleic Acids Res 39, 4577-4586.

Mooney, M., Bond, J., Monks, N., Eugster, E., Cherba, D., Berlinski, P., Kamerling, S., Marotti, K., Simpson, H., Rusk, T., et al. (2013). Comparative RNA-Seq and Microarray Analysis of Gene Expression Changes in B-Cell Lymphomas of <italic>Canis familiaris</italic>. PLoS ONE 8, e61088.

Mosher, R.A., Melnyk, C.W., Kelly, K.A., Dunn, R.M., Studholme, D.J., and Baulcombe, D.C. (2009). Uniparental expression of PollV-dependent siRNAs in developing endosperm of Arabidopsis. Nature 460, 283-286.

Narsai, R., Howell, K.A., Millar, A.H., O'Toole, N., Small, I., and Whelan, J. (2007). Genome-wide analysis of mRNA decay rates and their determinants in Arabidopsis thaliana. Plant Cell 19, 3418-3436.

Ohshima, K. (2013). RNA-Mediated Gene Duplication and Retroposons: Retrogenes, LINEs, SINEs, and Sequence Specificity. Int J Evol Biol 2013, 424726.

Okamura, K., and Nakai, K. (2008). Retrotransposition as a source of new promoters. Mol Biol Evol 25, 1231-1238. 
Onodera, Y., Haag, J.R., Ream, T., Costa Nunes, P., Pontes, O., and Pikaard, C.S. (2005). Plant nuclear RNA polymerase IV mediates siRNA and DNA methylation-dependent heterochromatin formation. Cell 120, 613-622.

Paradis, E. (2010). pegas: an R package for population genetics with an integrated-modular approach. Bioinformatics 26, 419-420.

Pei, B., Sisu, C., Frankish, A., Howald, C., Habegger, L., Mu, X., Harte, R., Balasubramanian, S., Tanzer, A., Diekhans, M., et al. (2012). The GENCODE pseudogene resource. Genome Biol 13, R51.

Pennisi, E. (2012). ENCODE project writes eulogy for junk DNA. Science 337, 1159-1161.

Pink, R.C., Wicks, K., Caley, D.P., Punch, E.K., Jacobs, L., and Carter, D.R. (2011). Pseudogenes: pseudo-functional or key regulators in health and disease? Rna 17, 792-798.

Popova, O.V., Dinh, H.Q., Aufsatz, W., and Jonak, C. (2013). The RdDM pathway is required for basal heat tolerance in Arabidopsis. Mol Plant 6, 396-410.

Potrzebowski, L., Vinckenbosch, N., Marques, A.C., Chalmel, F., Jégou, B., and Kaessmann, H. (2008). Chromosomal gene movements reflect the recent origin and biology of therian sex chromosomes. PLoS Biol 6, e80.

Quinlan, A.R., and Hall, I.M. (2010). BEDTools: a flexible suite of utilities for comparing genomic features. Bioinformatics 26, 841-842.

Ream, T.S., Haag, J.R., Wierzbicki, A.T., Nicora, C.D., Norbeck, A.D., Zhu, J.K., Hagen, G., Guilfoyle, T.J., Pasa-Tolic, L., and Pikaard, C.S. (2009). Subunit compositions of the RNA-silencing enzymes Pol IV and Pol V reveal their origins as specialized forms of RNA polymerase II. Molecular cell 33, 192-203.

Remm, M., Storm, C.E., and Sonnhammer, E.L. (2001). Automatic clustering of orthologs and in-paralogs from pairwise species comparisons. Journal of molecular biology 314, 10411052.

Roudier, F., Ahmed, I., Berard, C., Sarazin, A., Mary-Huard, T., Cortijo, S., Bouyer, D., Caillieux, E., Duvernois-Berthet, E., Al-Shikhley, L., et al. (2011). Integrative epigenomic mapping defines four main chromatin states in Arabidopsis. EMBO J 30, 1928-1938.

Roy, S.W., and Irimia, M. (2009). Mystery of intron gain: new data and new models. Trends in genetics : TIG 25, 67-73.

Sakai, H., Mizuno, H., Kawahara, Y., Wakimoto, H., Ikawa, H., Kawahigashi, H., Kanamori, H., Matsumoto, T., Itoh, T., and Gaut, B.S. (2011). Retrogenes in rice (Oryza sativa L. ssp. japonica) exhibit correlated expression with their source genes. Genome Biol Evol 3, 1357-1368. 
Sakharkar, M.K., Chow, V.T.K., and Kangueane, P. (2004). Distributions of exons and introns in the human genome. In Silico Biol 4, 387-393.

Schmid, M., Davison, T.S., Henz, S.R., Pape, U.J., Demar, M., Vingron, M., Scholkopf, B., Weigel, D., and Lohmann, J.U. (2005). A gene expression map of Arabidopsis thaliana development. Nat Genet 37, 501-506.

Seymour, D.K., Koenig, D., Hagmann, J., Becker, C., and Weigel, D. (2014). Evolution of DNA methylation patterns in the Brassicaceae is driven by differences in genome organization. PLoS Genet 10, e1004785.

Simillion, C., Janssens, K., Sterck, L., and Van de Peer, Y. (2008). i-ADHoRe 2.0: an improved tool to detect degenerated genomic homology using genomic profiles. Bioinformatics 24, 127-128.

Slotkin, R.K., Vaughn, M., Borges, F., Tanurdžić, M., Becker, J.D., Feijó, J.A., and Martienssen, R.A. (2009). Epigenetic reprogramming and small RNA silencing of transposable elements in pollen. Cell 136, 461-472.

Slotte, T., Hazzouri, K.M., Agren, J.A., Koenig, D., Maumus, F., Guo, Y.L., Steige, K., Platts, A.E., Escobar, J.S., Newman, L.K., et al. (2013). The Capsella rubella genome and the genomic consequences of rapid mating system evolution. Nat Genet 45, 831-835.

Song, W.-Y., Choi, K.S., Kim, D.Y., Geisler, M., Park, J., Vincenzetti, V., Schellenberg, M., Kim, S.H., Lim, Y.P., Noh, E.W., et al. (2010). Arabidopsis PCR2 is a zinc exporter involved in both zinc extrusion and long-distance zinc transport. Plant Cell 22, 2237-2252.

Stajich, J.E., Block, D., Boulez, K., Brenner, S.E., Chervitz, S.A., Dagdigian, C., Fuellen, G., Gilbert, J.G., Korf, I., Lapp, H., et al. (2002). The Bioperl toolkit: Perl modules for the life sciences. Genome Res 12, 1611-1618.

Stanke, M., Diekhans, M., Baertsch, R., and Haussler, D. (2008). Using native and syntenically mapped cDNA alignments to improve de novo gene finding. Bioinformatics 24 , 637-644.

Stanke, M., Tzvetkova, A., and Morgenstern, B. (2006). AUGUSTUS at EGASP: using EST, protein and genomic alignments for improved gene prediction in the human genome. Genome Biol 7 Supp/ 1, S11 11-18.

Stroud, H., Greenberg, Maxim V.C., Feng, S., Bernatavichute, Yana V., and Jacobsen, Steven E. (2013). Comprehensive analysis of silencing mutants reveals complex regulation of the Arabidopsis methylome. Cell 152, 352-364.

Swarbreck, D., Wilks, C., Lamesch, P., Berardini, T.Z., Garcia-Hernandez, M., Foerster, H., Li, D., Meyer, T., Muller, R., Ploetz, L., et al. (2008). The Arabidopsis Information 
Resource (TAIR): gene structure and function annotation. Nucleic Acids Res 36, D10091014.

Szczesniak, M.W., Ciomborowska, J., Nowak, W., Rogozin, I.B., and Makalowska, I. (2011). Primate and rodent specific intron gains and the origin of retrogenes with splice variants. Mol Biol Evol 28, 33-37.

Tsukahara, S., Kawabe, A., Kobayashi, A., Ito, T., Aizu, T., Shin-i, T., Toyoda, A., Fujiyama, A., Tarutani, Y., and Kakutani, T. (2012). Centromere-targeted de novo integrations of an LTR retrotransposon of Arabidopsis lyrata. Genes \& development 26, 705713.

Tucker, S.L., Reece, J., Ream, T.S., and Pikaard, C.S. (2010). Evolutionary history of plant multisubunit RNA polymerases IV and V: subunit origins via genome-wide and segmental gene duplications, retrotransposition, and lineage-specific subfunctionalization. Cold Spring Harb Symp Quant Biol 75, 285-297.

Vaucheret, H., and Fagard, M. (2001). Transcriptional gene silencing in plants: targets, inducers and regulators. Trends in genetics : TIG 17, 29-35.

Vinckenbosch, N., Dupanloup, I., and Kaessmann, H. (2006). Evolutionary fate of retroposed gene copies in the human genome. Proc Natl Acad Scie USA 103, 3220-3225.

Wang, W., Zheng, H., Fan, C., Li, J., Shi, J., Cai, Z., Zhang, G., Liu, D., Zhang, J., Vang, S., et al. (2006). High rate of chimeric gene origination by retroposition in plant genomes. Plant Cell 18, 1791-1802.

Wang, Y., and Ma, H. (2011). Development: a pathway to plant female germ cells. Current biology : CB 21, R476-478.

Yenerall, P., Krupa, B., and Zhou, L. (2011). Mechanisms of intron gain and loss in Drosophila. BMC Evol Biol 11, 364.

Yogeeswaran, K., Frary, A., York, T.L., Amenta, A., Lesser, A.H., Nasrallah, J.B., Tanksley, S.D., and Nasrallah, M.E. (2005). Comparative genome analyses of Arabidopsis spp.: inferring chromosomal rearrangement events in the evolutionary history of $A$. thaliana. Genome Res 15, 505-515.

Yoshida, S., Maruyama, S., Nozaki, H., and Shirasu, K. (2010). Horizontal gene transfer by the parasitic plant Striga hermonthica. Science 328, 1128.

Zhang, J. (2003). Evolution by gene duplication: an update. Trends Ecol Evol 18, 292-298.

Zhang, Y., Wu, Y., Liu, Y., and Han, B. (2005). Computational identification of 69 retroposons in Arabidopsis. Plant Physiol 138, 935-948. 
Zhao, S., Fung-Leung, W.-P., Bittner, A., Ngo, K., and Liu, X. (2014). Comparison of RNASeq and Microarray in Transcriptome Profiling of Activated T Cells. PLoS ONE 9, e78644.

Zhu, X., Ma, H., and Chen, Z. (2011). Phylogenetics and evolution of Su(var)3-9 SET genes in land plants: rapid diversification in structure and function. BMC Evol Biol 11, 1-11.

Zhu, Z., Zhang, Y., and Long, M. (2009). Extensive structural renovation of retrogenes in the evolution of the Populus genome. Plant Physiol 151, 1943-1951. 


\section{CURRICULUM VITAE}

PERSONAL INFORMATION

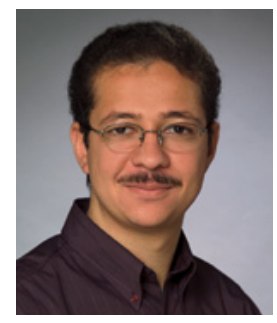

EDUCATION AND TRAINING

$2011-2014$

Oct. 2009 - Mar. 2011

Sept. 2003 - July 2007

WORK EXPERIENCE

Apr. 2012 - present

June 2008 - Mar. 2012

June 2007 - May 2008

RELATED PUBLICATIONS

\section{Ahmed Abdelsamad}
14 Kolibriweg, Cologne - 50829, Germany
自 +49 (0) 17635872796
ahmed.m.abdelsamad@gmail.com

Gender Male | Date of birth 06/07/1986 | Nationality Egyptian

$\mathrm{PhD}$ in Genome and Epigenome Evolution Group

Max Planck Institute for Plant Breeding Research / University of Göttingen, Germany.

-Thesis: Evolution and epigenetic regulation of RNA-duplicated genes in Arabidopsis

MSc in Molecular Biology

Max Planck Institute for Biophysical chemistry / University of Göttingen, Germany.

- Thesis: MicroRNA role in the regenerating pancreas of the mouse.

Honours BSc in Biotechnology

Faculty of Agriculture, Cairo University, Egypt.

- Genetics, biochemistry, microbiology and ecology.

Assistant lecturer

Department of Genetics, Faculty of Agriculture, Cairo University, Egypt.

Teaching and research assistant

Department of Genetics, Faculty of Agriculture, Cairo University, Egypt.

Research assistant

Agricultural genetic engineering research institute, Agricultural research center, Egypt.

V. Rawat, A. Abdelsamad, B. Pietzenuk, D. Seymour, D. Koenig, D. Weigel, A. Pecinka and K. Schneeberger. Improving the annotation of Arabidopsis lyrata using RNA-seq data 2015 (Submitted to BMC Genomics).

A. Abdelsamad, A. Pecinka. Pollen-specific transcription of Arabidopsis retrogenes is associat with global transcriptional reprogramming. (2014) The Plant Cell, vol. 26 (8), pp.3299-3313.

Göttingen, 28.05.2015

Signature 